\title{
Continuity and Convergence in Rational Triangular Bézier Spline Based Isogeometric Analysis
}

\author{
Songtao Xia, Xilu Wang, Xiaoping Qian* \\ Department of Mechanical Engineering, University of Wisconsin-Madison, \\ 1513 University Avenue, Madison, WI 53706
}

\begin{abstract}
This paper presents a method for isogeometric analysis using rational Triangular Bézier Splines (rTBS) where optimal convergence rates are achieved. In this method, both the geometry and the physical field are represented by bivariate splines in Bernstein Bézier form over the triangulation of a domain. From a given physical domain bounded by NURBS curves, a parametric domain and its triangulation are constructed. By imposing continuity constraints on Bézier ordinates, we obtain a set of global $C^{r}$ smooth basis functions. Convergence analysis shows that isogeometric analysis with such $C^{r}$ rTBS basis can deliver the optimal rate of convergence provided that the $C^{r}$ geometric map remains unchanged during the refinement process. This condition can be satisfied by constructing a pre-refinement geometric map that is sufficiently smooth. Numerical experiments verify that optimal rates of convergence are achieved for Poisson and linear elasticity problems.
\end{abstract}

Keywords: isogeometric analysis, triangular Bézier spline, geometric map, optimal convergence rate

\section{Introduction}

Isogeometric analysis is an analysis approach introduced by Hughes et al [1] where the same basis functions used to represent geometric models, such as Non-Uniform Rational B-Splines (NURBS), are also used to approximate field variables in solving partial differential equations (PDEs). Due to the same basis used in geometric representation and in solution approximation, it eliminates the geometric approximation error commonly occurred in classical finite element analysis (FEA) procedures. Once the initial mesh is constructed, refinements can also be easily implemented and exact geometry is maintained at all levels without the necessity of interaction with the CAD system [1, 2]. Such ability to represent exact shape with a coarse mesh has also led to the development of isogeometric shape optimization [3, 4, 5]. Another advantage of isogeometric analysis is its computational efficiency on a per-node basis over classical $C^{0}$ Lagrange polynomial based finite element. The higher continuity of the NURBS basis has been demonstrated to significantly improve the numerical efficiency and accuracy on a per node basis in many areas including structural analysis [2, 6], fluid simulation [7] and shape optimization [4, 5, 8].

Isogeometric analysis techniques based on basis other than NURBS have also been developed. To overcome the limitation of the tensor product structure of NURBS in

\footnotetext{
${ }^{*}$ Corresponding author.

Email address: qian@engr.wisc.edu (Xiaoping Qian)
} 
local mesh refinement, methods based on subdivision solids [9] and T-splines [10, 11, 12, 13. have been developed recently and successfully used in isogeometric analysis. The introduction of T-junction in T-splines allows it to represent complex shapes in a single patch and permit local refinement [14]. On the other hand, challenges with analysis suitable T-splines [13, 14, 15] include how to obtain efficient local refinement and and effective treatment of so-called extraordinary points [12].

Recently triangular Bézier splines have emerged as a powerful alternative to shape modeling and isogeometric analysis due to their flexibility in representing domains of complex topology and their higher order of continuity. Local refinement can also be implemented without any difficulty. Bivariate splines of various degrees and smoothness have been used for data fitting and numerical solution of partial differential equations on simple geometries such as rectangular and circular domain [16, 17, 18, 19, 20]. In these work, the smoothness conditions are treated as side constraints and enforced through Lagrange multipliers. In [21] piecewise quadratic $C^{1}$ polynomial known as Powell-Sabin (PS) spline is introduced. Early work of PS splines can be found in the field of approximation in [22, 23, 24]. Later normalized basis of PS splines with convex partition of unity is studied in [25, 26, 27] and used for numerical solution of partial differential equations [28]. The PS splines can be extended to higher degrees with higher order of smoothness [33, 34, 35, 36]. Normalized basis for other splines such as reduced Clough-Tocher (CT) splines have also been investigated [37]. Further studies of quadratic $C^{1}$ PS splines in the context of IGA can be found in [29, 30, 31, 32]. However, the conversion of NURBS geometries (the de factor representation in CAD systems) to PS splines representation is not trivial and some NURBS surfaces even cannot be reproduced by PS splines [30]. There is no reported study on the optimal convergence behavior with such PS B-splines in IGA of general geometries.

In [38, a generalized framework of IGA based on rational triangular Bézier splines (rTBS) which is compatible with any form of bivariate $C^{r}$ Bézier elements is introduced. A globally $C^{r}$ continuous basis for representing both physical field and domain geometry with exact recovery of its NURBS boundary can be constructed in different spaces, including polynomial macro-element spaces, PS macro-element spaces and CT macro-element spaces.

The main idea of rTBS based IGA is illustrated in Figure 1 where cubic $C^{1}$ smooth basis functions with CT macro-elements are used as an example. The given physical domain is triangulated into a set of $C^{1}$ smooth Bézier elements, which are mapped from the parametric mesh. The $C^{1}$ basis functions are constructed as linear combinations of the $C^{0}$ Bernstein basis, under the continuity constraints. The resulting analysis has shown to be efficient, accurate and convergent. However, optimal convergence in $h$-refinement has only been achieved for $C^{0}$ elements and the convergence rate is sub-optimal for $C^{r}$ elements.

In this paper we present an approach that can lead to optimal convergence for all $C^{r}$ rTBS elements based isogeometric analysis in the context of $h$-refinement. Approximation power and convergence rates are widely used to evaluate the performance of numerical schemes for solving PDEs. The NURBS space has been proved to have full approximation power and deliver the optimal rate of convergence [39] as the classical finite element spaces. Similar approximation estimates which are optimal with respect to the polynomial degree of the underlying spline space have also been developed for T-splines that are defined on a particular two-patch structure [40]. However, no convergence results have been reported for T-splines defined on more generalized T-meshes that 
include extraordinary points, which usually require additional continuity constraints on the surrounding control points to achieve $G^{1}$ continuity [41, 42].

In our earlier approach to rTBS based isogeometric analysis [38], the initial coarse parametric mesh is first refined into elements that are sufficiently small for analysis, the global $C^{r}$ smooth basis is then constructed by imposing continuity constraints on adjacent triangles in the refined parametric mesh. Based on the $C^{r}$ basis, the $C^{r}$ geometric map is obtained. Although such a refine-then-smooth approach does lead to a $C^{r}$ stable basis that is sufficient for analysis, the resulting geometric map may not be consistent after the $C^{r}$ constraints are imposed. The inconsistency in geometric maps leads to deteriorated converged rate. The reason for such inconsistency in geometric map is as follows. The $C^{r}$ basis is obtained via continuity constraints on domain points in the parametric mesh. With the $C^{r}$ basis, some domain points are free and other domain points are dependent on these free points. For the geometric map that maps the parametric mesh to the physical mesh, the control points corresponding to the free domain points are chosen as free control points. If the remaining dependent control points do not satisfy the same continuity constraints, they would have to be relocated to satisfy the constraints to ensure the map is $C^{r}$. Such relocation of dependent control points thus lead to a change of the geometric map.

In this paper, to overcome such inconsistency of geometric map in $h$-refinement with the refine-then-smooth approach, we introduce a three-step approach to achieve optimal convergence in IGA with $C^{r}$ rTBS elements. We first construct a pre-refinement geometric map that possesses sufficient smoothness to maintain the consistency of the geometric map for all subsequent refinements. From the pre-refinement smooth geometric map, we uniformly refine the mesh. We then use macro-element techniques to obtain stable $C^{r}$ basis for analysis. In such a smooth-refine-smooth approach, the smoothness in the first step is needed for purely geometric reason so that the resulting geometric map stays consistent during the mesh refinement. The smoothness in the last step is to ensure stable basis over triangulation for analysis. With such a smooth-refine-smooth approach, we show optimal convergence in $h$-refinement can be achieved for all types of $C^{r}$ rTBS elements.

We further demonstrate this approach is also applicable to supersplines $S_{d}^{r, \rho},(\rho>r)$, where some vertices or edges in macro-triangles possess higher order $C^{\rho}$ smoothness than the global $C^{r}$ smoothness. In such cases, the smoothness of the pre-refinement geometric map should be $C^{\rho}$.

The remainder of this paper begins with a brief introduction of triangular Bézier splines in Section 2. The rTBS based parametrization is presented in Section 3. Section 4 describes the details of rTBS based isogeometric analysis. We then discuss the conditions to achieve optimal convergence rates in Section 5 followed by procedures to construct a consistent pre-refinement smooth geometric map in Section 6. Some numerical results are presented in Section 7. Finally we draw the conclusions in Section 8.

\section{Triangular Bézier splines}

For the sake of completeness, in this section we briefly introduce the Bézier triangles and bivariate splines on triangulations. Interested readers may see [33] and [43] for further details. 
- Free control points $\mathbf{p}_{\mathbf{i}}^{f}$ (or domain points $\mathbf{q}_{\mathbf{i}}^{f}$ )

$\circ$ Dependent control points $\mathbf{p}_{\mathbf{i}}^{d}$ (or domain points $\mathbf{q}_{\mathbf{i}}^{d}$ )

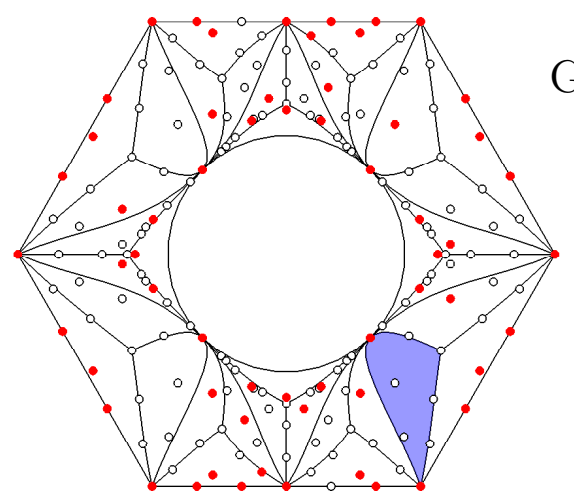

Physical mesh with

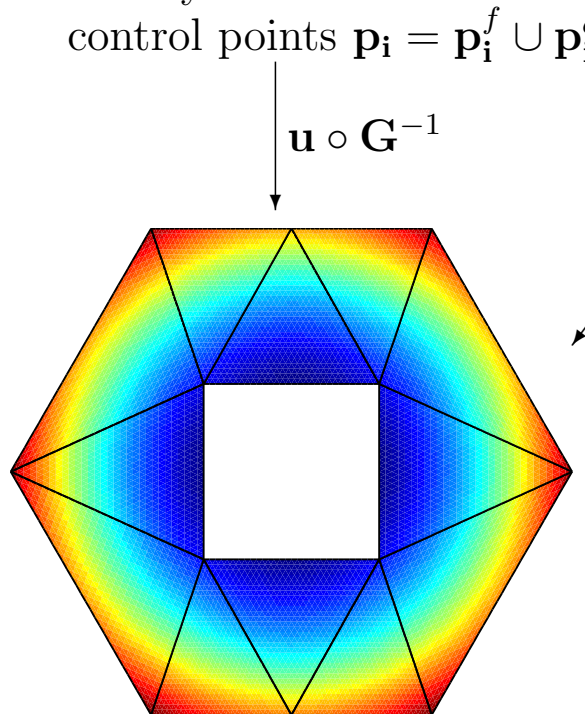

Field approximation
Geometrical map

$$
\frac{\mathbf{G}=\sum_{\mathbf{i}} \mathbf{p}_{\mathbf{i}}^{f} \psi_{\mathbf{i}}}{\mathbf{G}^{-1}}
$$

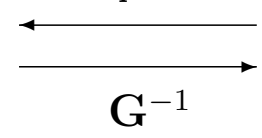

$$
\mathbf{p}_{\mathbf{i}}^{d}
$$

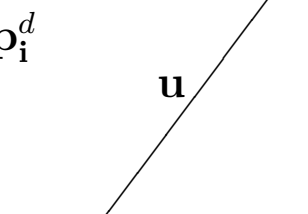

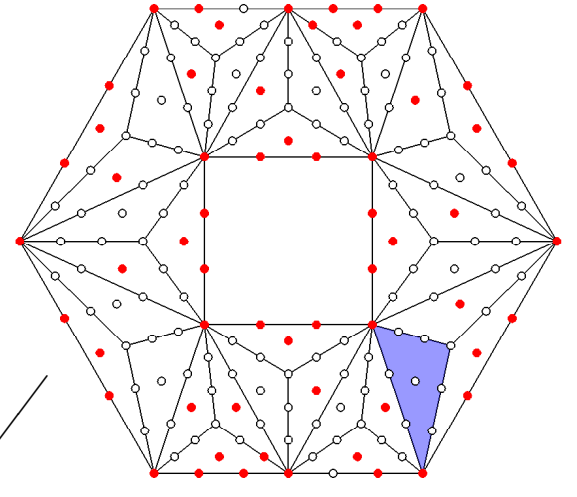

Parametric mesh with domain points $\mathbf{q}_{\mathbf{i}}=\mathbf{q}_{\mathbf{i}}^{f} \cup \mathbf{q}_{\mathbf{i}}^{d}$

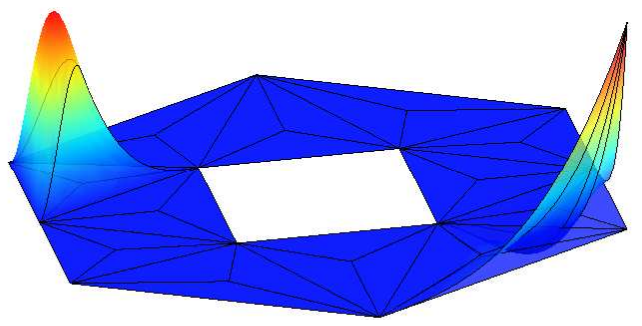

Basis functions $\boldsymbol{\psi}=\mathbf{C} \boldsymbol{\phi}$

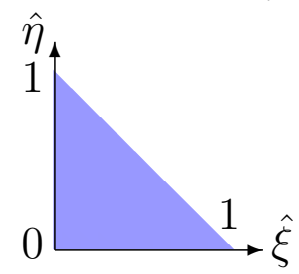

Integration on parent element

Figure 1: Schematic illustration of rTBS based isogeometric analysis. The red solid control points and domain points are determined by the white empty control points and domain points respectively under the continuity constraints. Cubic $C^{1}$ continuous basis functions with Clough-Tocher macro-elements are used as an example. The $C^{1}$ continuous basis $\boldsymbol{\psi}$ is a linear combination of the $C^{0}$ Bernstein basis $\phi$. 


\subsection{Bézier triangles}

NURBS has been widely used as a standard to represent curves and surfaces in CAD systems. Each knot span of a NURBS curve corresponds to a Bézier curve which is defined through Bernstein basis functions. A $d$-th degree Bernstein polynomial is defined as

$$
B_{i j, d}(\xi)=\left(\begin{array}{c}
d \\
i, j
\end{array}\right) \xi^{i}(1-\xi)^{d-i}, \quad \xi \in[0,1],
$$

where $\left(\begin{array}{c}d \\ i, j\end{array}\right)=\frac{d !}{i ! j !}, i+j=d$. Accordingly a $d$-th degree bivariate Bernstein polynomial is defined as

$$
B_{\mathbf{i}, d}(\boldsymbol{\xi})=\frac{d !}{i ! j ! k !} \gamma_{1}^{i} \gamma_{2}^{j} \gamma_{3}^{k}, \quad|\mathbf{i}|=i+j+k=d,
$$

where $\mathbf{i}$ represents a triple index $(i, j, k)$ and $\left(\gamma_{1}, \gamma_{2}, \gamma_{3}\right)$ is the barycentric coordinate of a point $\boldsymbol{\xi} \in \mathbb{R}^{2}$. Every point $\boldsymbol{\xi}=\left(\xi_{1}, \xi_{2}\right)$ in a fixed triangle with vertices $\mathbf{v}_{1}, \mathbf{v}_{2}, \mathbf{v}_{3} \in \mathbb{R}^{2}$ can be written uniquely in the form

$$
\boldsymbol{\xi}=\gamma_{1} \mathbf{v}_{1}+\gamma_{2} \mathbf{v}_{2}+\gamma_{3} \mathbf{v}_{3}
$$

with

$$
\gamma_{1}+\gamma_{2}+\gamma_{3}=1
$$

It has been shown that the set $\left\{B_{\mathbf{i}, d}\right\}_{|\mathbf{i}|=d}$ is a basis for the space of degree $d$ bivariate polynomials $\mathcal{P}_{d}$ [33]. A triangular Bézier patch is defined as

$$
\mathbf{b}(\boldsymbol{\xi})=\sum_{|\mathbf{i}|=d} \mathbf{p}_{\mathbf{i}} B_{\mathbf{i}, d}(\boldsymbol{\xi}),
$$

where $\mathbf{p}_{\mathbf{i}}$ represents a triangular array of control points. A rational Bézier triangle can be defined similarly as

$$
\mathbf{b}(\boldsymbol{\xi})=\sum_{|\mathbf{i}|=d} \mathbf{p}_{\mathbf{i}} \phi_{\mathbf{i}, d}(\boldsymbol{\xi}),
$$

with $\phi_{\mathbf{i}, d}$ being the rational Bernstein basis

$$
\phi_{\mathbf{i}, d}=\frac{w_{\mathbf{i}} B_{\mathbf{i}, d}}{\sum_{|\mathbf{i}|=d} w_{\mathbf{i}} B_{\mathbf{i}, d}}=\frac{w_{\mathbf{i}} B_{\mathbf{i}, d}}{w},
$$

where $w_{\mathbf{i}}$ are the weights associated with the control points $\mathbf{p}_{\mathbf{i}}$.

Under the isoparametric concept, the same bivariate Bernstein basis defining a triangle $\tau=\left\{\mathbf{v}_{1}, \mathbf{v}_{2}, \mathbf{v}_{3}\right\}$ can also be used to define a polynomial function $f$ of degree $d$ over $\tau$ as

$$
f(\boldsymbol{\xi})=\sum_{|\mathbf{i}|=d} b_{\mathbf{i}} \phi_{\mathbf{i}, d}(\boldsymbol{\xi}) .
$$

The $b_{\mathbf{i}}\left(\right.$ or $\left.b_{i j k}\right)$ are called the Bézier ordinates of $f$. Their associated set of domain points are defined as

$$
\mathcal{D}_{d, \tau}=\left\{\mathbf{q}_{i j k}=\frac{i \mathbf{v}_{1}+j \mathbf{v}_{2}+k \mathbf{v}_{3}}{d}, i+j+k=d\right\} .
$$

Thus the control polygon of the function $f$ is given by the points $\left(\mathbf{q}_{i j k}, b_{i j k}\right)$. Figure 2 gives an example of the associated domain points of the Bézier ordinates and triangular Bézier patch. 


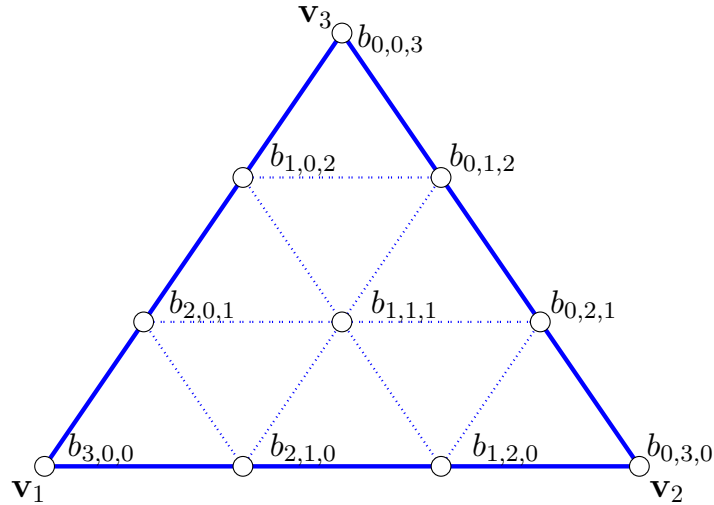

(a) Associated domain points of the Bézier ordinates $b_{i j k}$ in $\left\{\mathbf{v}_{1}, \mathbf{v}_{2}, \mathbf{v}_{3}\right\}$.

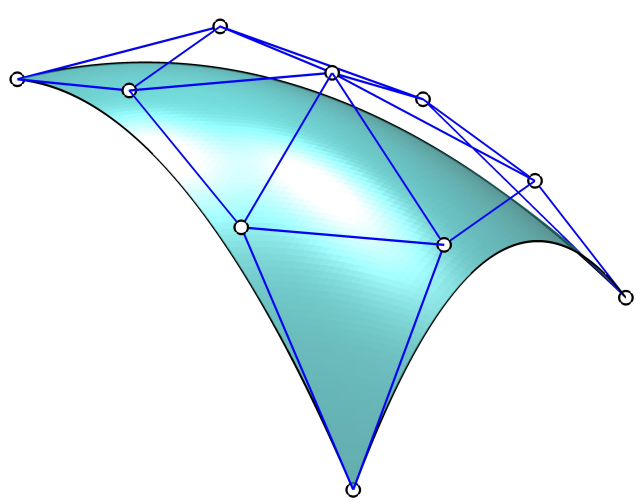

(b) Triangular Bézier patch $\mathbf{b}(\boldsymbol{\xi})$.

Figure 2: Domain points and triangular Bézier patch.

Two polynomials $f$ and $\tilde{f}$ of degree $d$ join $r$ times differentiably across the common edge of two triangles $\tau=\left\{\mathbf{v}_{1}, \mathbf{v}_{2}, \mathbf{v}_{3}\right\}$ and $\tilde{\tau}=\left\{\mathbf{v}_{4}, \mathbf{v}_{3}, \mathbf{v}_{2}\right\}$ if and only if 33]

$$
\tilde{b}_{\rho, j, k}-\sum_{\mu+\nu+\kappa=\rho} \frac{\rho !}{\mu ! \nu ! \kappa !} b_{\mu, k+\nu, j+\kappa} \gamma_{1}^{\mu} \gamma_{2}^{\nu} \gamma_{3}^{\kappa}=0, \quad j+k+\rho=d, \rho=0, \cdots, r,
$$

where $\gamma_{1}, \gamma_{2}, \gamma_{3}$ are the barycentric coordinates of vertex $\mathbf{v}_{4}$ with respect to triangle $\tau$. Figure 3 gives an illustration of two triangular Bézier patches with $C^{1}$ continuity constraints. The red solids represent free nodes whose values can be freely chosen, while the three white solids representing dependent nodes are determined by the red free nodes through the continuity constraints. The shaded area indicates the triangles where continuity constraints are imposed. As can be seen in Figure 3(b), the control points in each shaded triangle pair are coplanar. For better visualization of the underlying $C^{1}$ patch, the control net in Figure 3(b) is shifted up slightly.

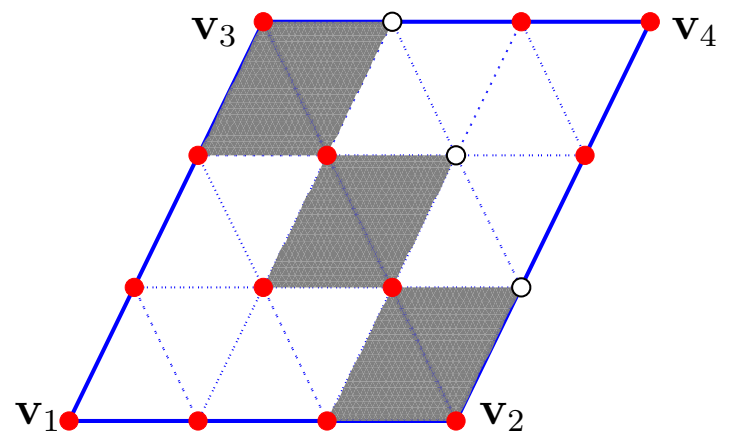

(a) Two domain triangles with $C^{1}$ constraints on Bézier ordinates.

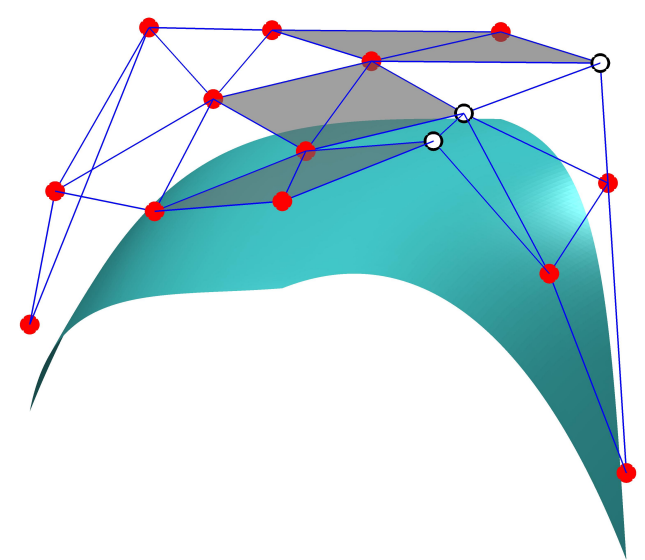

(b) Two Bézier patches with $C^{1}$ continuity.

Figure 3: Triangular Bézier patches with $C^{1}$ continuity. The dependent nodes (white solids) are determined by the free nodes (red solids) through the continuity constraints. The shaded areas indicate the triangles with shared edges where the constraints are imposed. As can be seen in Figure 3(b), the control points in each shaded triangle pair are coplanar. For better visualization, the control net is shifted up slightly in Figure $3(\mathrm{~b})$, 


\subsection{Splines on triangulations}

Consider a parametric domain $\widehat{\Omega}$ and its triangulation $\widehat{T}$. We introduce the spline spaces of piecewise polynomials of degree $d$ over $\widehat{T}[33]$

$$
\mathcal{S}_{d}^{r}(\widehat{T})=\left\{f \in C^{r}(\widehat{\Omega}):\left.f\right|_{\tau} \in \mathcal{P}_{d} \forall \tau \in \widehat{T}\right\},
$$

where $\tau$ is an arbitrary triangle in $\widehat{T}$ and $r$ is the continuity order of the spline over $\widehat{\Omega}$. In addition, if the spline has higher smoothness at some vertices or across some edges, we call it a superspline and denote the associated space as [33]

$$
\mathcal{S}_{d}^{r, \rho}(\widehat{T})=\left\{f \in \mathcal{S}_{d}^{r}(\widehat{T}): f \in C^{\rho_{\mathbf{v}}}(\mathbf{v}) \forall \mathbf{v} \in \mathbf{V} \& f \in C^{\rho_{\mathbf{e}}}(\mathbf{e}) \forall \mathbf{e} \in \mathbf{E}\right\},
$$

where $\mathbf{V}$ and $\mathbf{E}$ are the set of all vertices and edges respectively in $\widehat{T}$ and $\rho:=\left\{\rho_{\mathbf{v}}\right\}_{\mathbf{v} \in \mathbf{V}} \cup$ $\left\{\rho_{\mathbf{e}}\right\}_{\mathbf{e} \in \mathbf{E}}$ with $r \leq \rho_{\mathbf{v}}, \rho_{\mathbf{e}} \leq d$ for each $\mathbf{v} \in \mathbf{V}$ and $\mathbf{e} \in \mathbf{E}$.

There are several approaches to obtain $C^{r}$ spline spaces on a triangulated domain $\widehat{\Omega}(\widehat{T})$. In this paper we are interested in the spaces $\mathcal{S}_{d}^{r}$ and $\mathcal{S}_{d}^{r, \rho}$ with full approximation power of $d$-th degree polynomials. The straightforward way is to apply condition (9) directly on the triangles, which requires the degree of the polynomial much higher than $r$, such as $d \geq 3 r+2$ [44]. The alternative way is splitting each triangle in $\widehat{T}$ into several micro-triangles before imposing the continuity constraints on the micro-triangles. The original triangles are then called macro-triangles. These include the CT split [45] with polynomials of degree $d \geq 3 r$ for continuity $r$-odd and $d \geq 3 r+1$ for $r$-even, and the PS split [21] with polynomials of degree $d \geq \frac{9 r-1}{4}$ for $r$-odd and of degree $d \geq \frac{9 r+4}{4}$ for $r$-even. For example, in this paper we use CT split to obtain $\mathcal{S}_{3}^{1}$ spline space with cubic polynomials, and PS split to obtain $\mathcal{S}_{2}^{1}, \mathcal{S}_{5}^{2}$ and $\mathcal{S}_{5}^{2,3}$ spline spaces with quadratic and quintic polynomials respectively. We also use so-called polynomial macro-element technique to obtain $\mathcal{S}_{5}^{1}$ and $\mathcal{S}_{5}^{1,2}$ spline spaces with quintic polynomials without using any split technique.

Figure 4 shows the CT and PS splits with corresponding free and dependent domain points respectively. In the CT split, each vertex of a triangle in $\widehat{T}$ is connected with its centroid point to form three micro-triangles, as shown in Figure 4(a). We denote this resulting triangulation as $\widehat{T}_{c t}$. In the PS split, for each triangle we connect its incenter to each of the three vertices and connect the two incenters of two triangles sharing a common edge. In addition, we connect the middle of each boundary edge to the incenter of the associated triangle, resulting in six micro-triangles. For the ease of implementation in this paper, the centroid point instead of the incenter of each triangle is used as the interior split point and the resulting triangulation is denoted as $\widehat{T}_{p s}$, as shown in Figure 4(b).

Uniform refinement can also be performed as needed. For example, each triangle can be subdivided into four sub-triangles by connecting the middle points of the edges. This kind of 1-to-4 split based uniform refinement is used in our subsequent analysis of convergence during mesh refinement.

\section{Domain parametrization with $C^{r}$ rTBS}

In this section we present our method for discretizing a physical domain $\Omega$ into a collection of rational Bézier triangles without any geometric approximation error.

Given an arbitrary 2D domain $\Omega$ and its NURBS-represented boundary $\Gamma$, we seek a geometric map $\mathbf{G}(\boldsymbol{\xi}), \boldsymbol{\xi} \in \widehat{\Omega}$ such that the physical domain $\Omega$ is the image of the geometric 


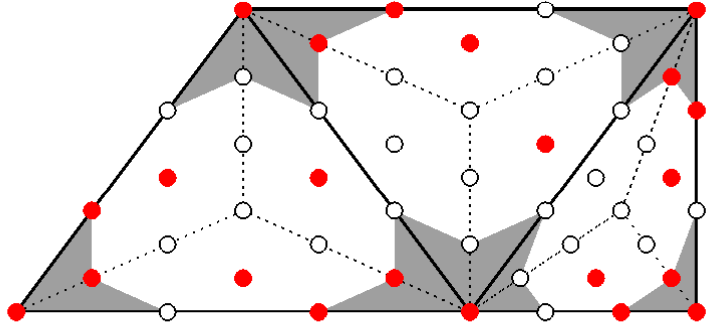

(a) Cubic $C^{1}$ mesh using CT split.

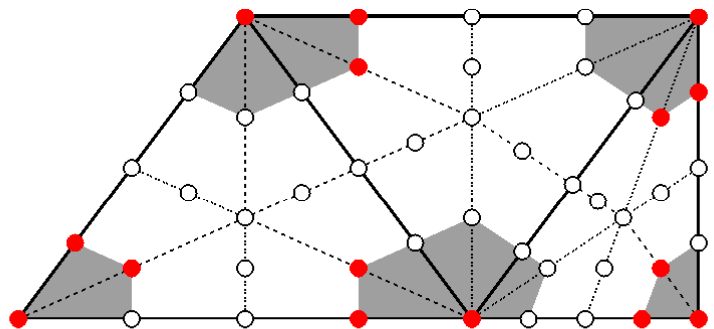

(b) Quadratic $C^{1}$ mesh using PS split.

Figure 4: The CT and PS refinements of macro-triangles with $C^{1}$ continuity constraints. The dependent domain points (white solids) are determined by the free domain points (red solids) through the continuity constraints. The shaded areas indicate where the continuity constraints are imposed.

map $\mathbf{G}(\boldsymbol{\xi})$ over a parametric domain $\widehat{\Omega}$ where the physical boundary is exactly reproduced by the map. In addition, the geometric map $\mathbf{G}(\boldsymbol{\xi})$ is continuous and differentiable up to any desired degree of continuity $C^{r}$. This can be achieved in three main steps as described below:

(i) Form a polygonal parametric domain $\widehat{\Omega}$ and its triangulation $\widehat{T}$ from the given physical domain $\Omega$ and the triangulation $T_{0}$. Establish a $C^{0}$ geometric map $\mathbf{G}_{0}$ between them: $\mathbf{G}_{0}: \widehat{\Omega}_{\widehat{T}} \mapsto \Omega_{T_{0}}$.

(ii) Construct a set of $C^{r}$ basis $\boldsymbol{\psi}(\boldsymbol{\xi})$ on $\widehat{\Omega}_{\widehat{T}}$.

(iii) Construct a $C^{r}$ continuous triangulation $T$ of $\Omega$ and establish a globally $C^{r}$ geometric map $\mathbf{G}(\boldsymbol{\xi})$ from the parametric domain $\widehat{\Omega}_{\widehat{T}}$ to the physical domain $\Omega_{T}$.

In the following subsections we explain in detail on each step. The method was initially proposed in [38], here we add an additional step (step (4) in Section 3.1) to avoid possible self-intersection in the physical mesh.

\subsection{Construction of a $C^{0}$ geometric map $\mathbf{G}_{0}$}

The process is illustrated in Figure 5 and can be described as follows.

(1) Given a domain $\Omega$ with NURBS boundary curves of degree $d$ (Figure $5(\mathrm{a})$ ), we subdivide each NURBS curve into a set of Bézier curves via knot insertions (Figure $5(\mathrm{~b})$.

(2) The end points of these Bézier curves are connected to form a polygonal parametric domain $\widehat{\Omega}$. The domain $\widehat{\Omega}$ is then triangulated using Delaunay triangulation to obtain $\widehat{T}_{0}$ and the associated domain points are generated according to Eq. (8) (Figure $5(\mathrm{~d})$ ).

(3) In order to reproduce the exact NURBS boundary in later $C^{r}$ parametrization, we further adjust those vertices in $\widehat{T}_{0}$ that correspond to $C^{k}(k \geq r)$ knot points in the physical boundary. We move each such boundary vertex to the line segment connecting the adjacent vertices of $C^{q}(0 \leq q<r)$ smoothness, and so that the length-ratio of consecutive edges is the same as the ratio of the corresponding knot intervals in the NURBS boundary curve. For example, in Figure $5(\mathrm{~d})$ and $5(\mathrm{e})$,

$$
\left|\mathbf{v}_{0} \mathbf{v}_{1}\right|:\left|\mathbf{v}_{1} \mathbf{v}_{2}\right|:\left|\mathbf{v}_{2} \mathbf{v}_{3}\right|:\left|\mathbf{v}_{3} \mathbf{v}_{4}\right|=\left(t_{1}-t_{0}\right):\left(t_{2}-t_{1}\right):\left(t_{3}-t_{2}\right):\left(t_{4}-t_{3}\right) .
$$


If there are too few $C^{q}$ knots points in the boundary curve to form a suitable domain $\widehat{\Omega}$, some smooth knot points (such as the two points marked as red squares in the inner circular boundary in Figure $5(\mathrm{a})$ are also used as corner points to form the polygonal domain (Figure 5(c)). In this way, we obtain a $C^{0}$ parametrization with $C^{r}$ smoothness along the boundary except at corner vertices where the smoothness is $C^{0}$ [38. The quality of the parametric mesh may also be improved by using appropriate techniques such as the smoothing method in [38.

(4) Replace the boundary control points of $\widehat{\Omega}_{\widehat{T}}$ with corresponding control points of the Bézier curves in the physical domain to obtain a triangulation $T_{0}$ on the physical domain (Figure 5(e)).

(5) Check the physical mesh $T_{0}$ to see if there is self-folding. If so (see Figure $5(\mathrm{j})$ for example, because of the over recessed control points, the control polygon of the curved boundary intersects with the other two boundaries of the element, causing self-folding of the mesh.), we subdivide the Bézier boundary curve where self-folding occurs and repeat steps (2)-(3) until a valid physical mesh $T_{0}$ is obtained (Figure $5(\mathrm{f}), 5(\mathrm{~g}), 5(\mathrm{i})$.

Alternatively, after replacing the boundary control points, we can apply mesh moving technique where the movement of the control points is governed by the elastic equation [46]. The resulting mesh by applying the mesh moving technique of mesh in Figure $5(\mathrm{e})$ is shown in Figure 5(o), where the self-folding has disappeared. Although the mesh moving technique does not guarantee a valid mesh, it works quite well in our practice. Better domain parametrization can be obtained by optimization techniques such as in [32].

To check the quality of the mesh, the determinant of the Jacobian of the meshes are calculated as

$$
\operatorname{det}(J)=\operatorname{det}\left[D_{0,1,-1}^{1} \mathbf{b}(\boldsymbol{\xi}), D_{-1,0,1}^{1} \mathbf{b}(\boldsymbol{\xi})\right],
$$

where $D_{0,1,-1}^{1} \mathbf{b}(\boldsymbol{\xi})$ and $D_{-1,0,1}^{1} \mathbf{b}(\boldsymbol{\xi})$ are the first order directional derivatives of $\mathbf{b}(\boldsymbol{\xi})$ in directions $(0,1,-1)$ and $(-1,0,1)$ respectively [33]. In Figure 5(l), negative Jacobian determinant can be seen, indicating self-folding occurs. After using either subdivision or mesh moving technique, the Jacobian determinant all becomes positive, as shown in Figure $5(\mathrm{n})$ and $5(\mathrm{p})$ respectively, which means there is no self-folding.

Note that the $C^{r}$ knot points on the physical boundary, after mapped to $C^{0}$ corner points on the parametric domain, become singular points. An example of such $C^{r}$ knot points is the end points of the circular Bézier segments in Figure 5(b). In order to alleviate such singularity issue, the mesh moving technique is always recommended instead of the subdivision method to produce the physical mesh.

\subsection{Construction of $C^{r}$ spline basis $\boldsymbol{\psi}(\boldsymbol{\xi})$ on $\widehat{T}$}

Let $\mathcal{D}_{d, \widehat{T}}$ denote the set of domain points for triangulation $\widehat{T}$ as defined in $(8), \mathbf{v} \in \mathcal{D}_{d, \widehat{T}}$ a domain point and $b_{\mathbf{v}}$ its ordinate. A piecewise polynomial function $f(\boldsymbol{\xi}) \in \mathcal{S}_{d}, \boldsymbol{\xi} \in \mathbb{R}^{2}$ can be expressed in terms of the rational $C^{0}$ Bernstein basis $\phi$ and corresponding nodal ordinates $\mathbf{b}_{\mathcal{D}_{d, \widehat{T}}}$ as

$$
f(\boldsymbol{\xi})=\sum_{i} b_{i} \phi_{i}(\boldsymbol{\xi})=\mathbf{b}_{\mathcal{D}_{d, \widehat{T}}^{T}}^{T} \boldsymbol{\phi}(\boldsymbol{\xi})
$$




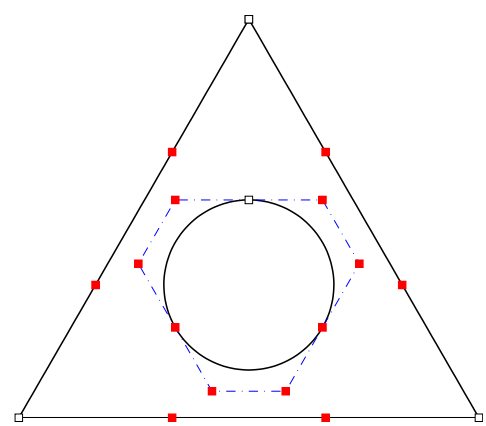

(a) Input domain with NURBS boundary.

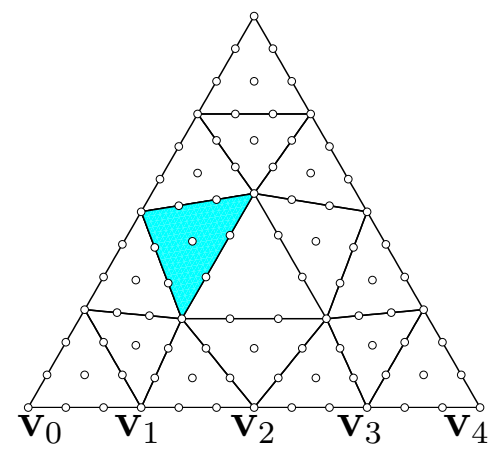

(d) Parametric mesh with domain points. $\mathbf{v}_{i}$ are boundary vertices corresponding to $C^{1}$ knots points in $5(\mathrm{e})$.

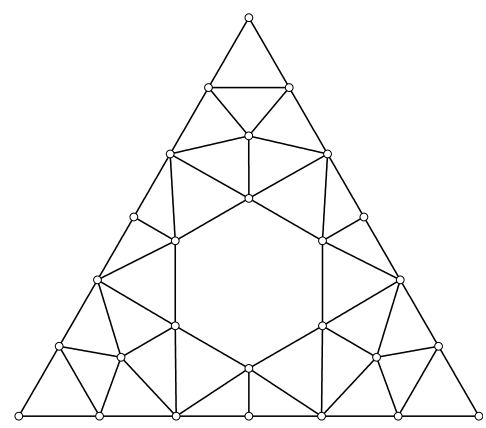

(g) New parametric mesh.

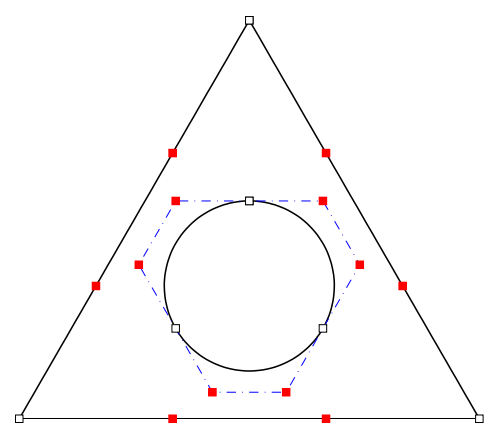

(b) Bézier extraction

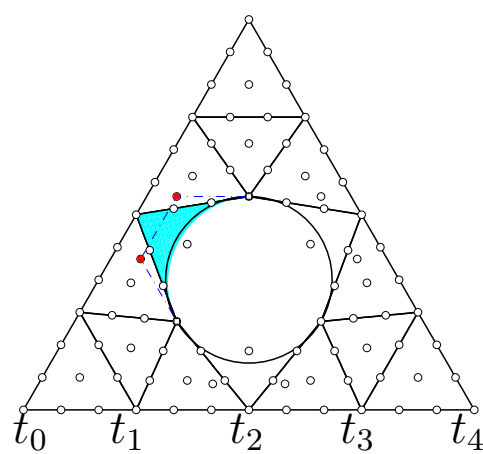

(e) Physical mesh after boundary replacement. $t_{i}$ are knot values of corresponding $C^{1}$ knot points.

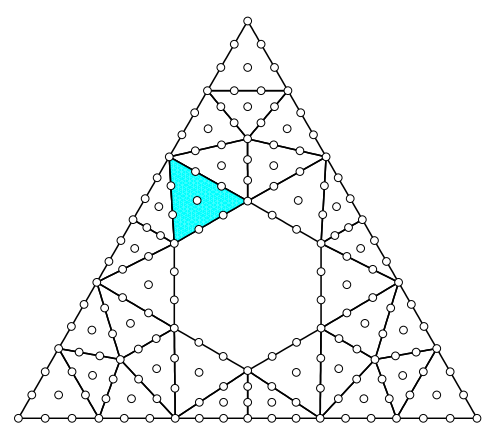

(h) New parametric mesh.

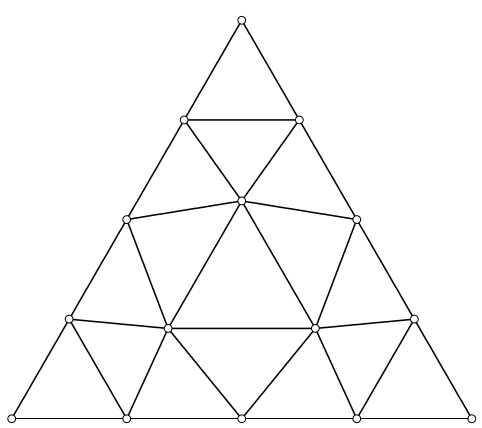

(c) Polygonal parametric mesh.

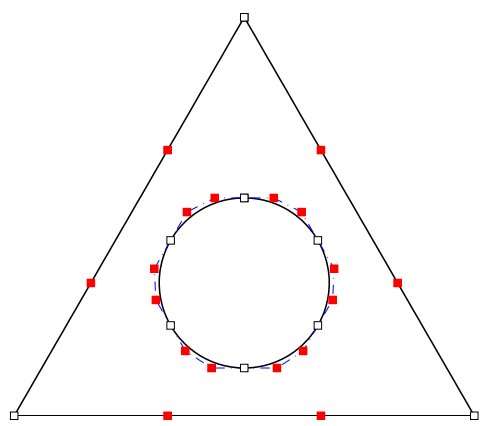

(f) Local Subdivision on the circle.

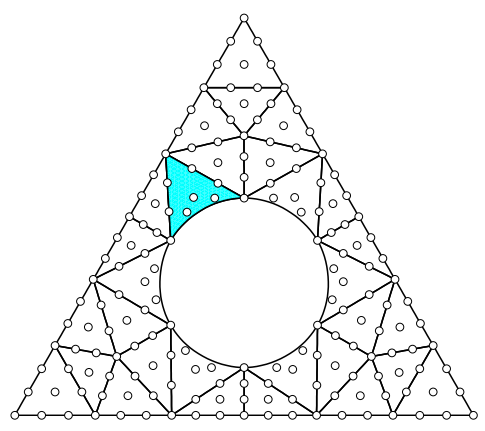

(i) New physical mesh after boundary replacement.

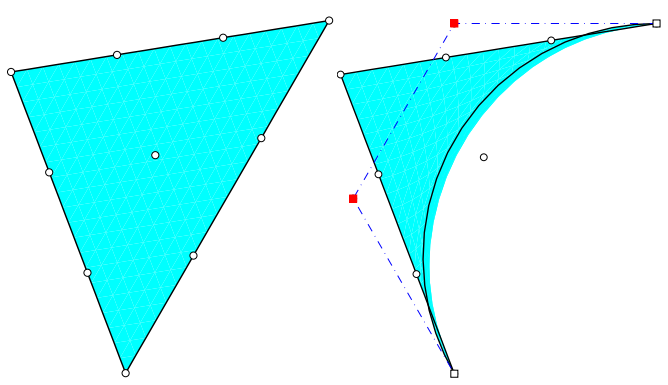

(j) Detailed view of Figure (e). Because of the over recessed control points, the control polygon of the curved boundary intersects with the other two boundaries of the element, causing self-folding of the mesh.

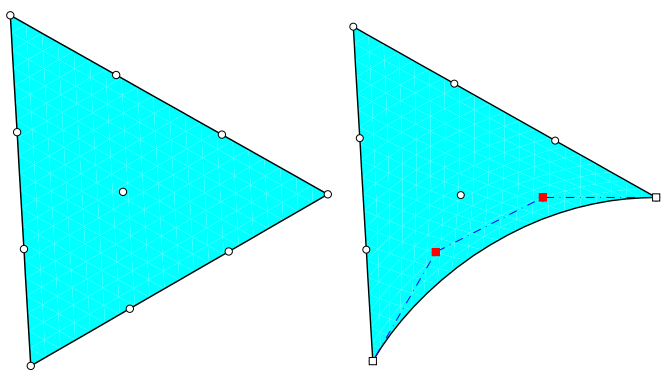

(k) Detailed view of Figure (i). The control polygon of the curved boundary is contained between the other two boundaries of the element, the mesh is clear of self-folding. 


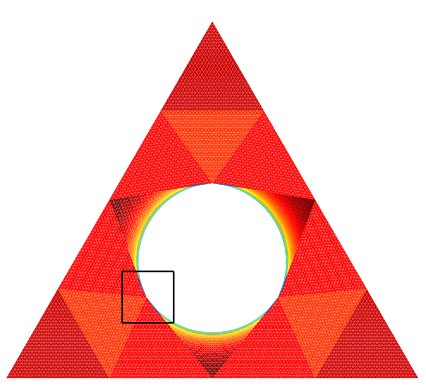

(l) Jacobian determinant of mesh in Figure 5(e)

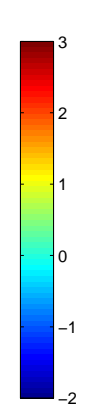

(m) Zoom-in view of the folded area.

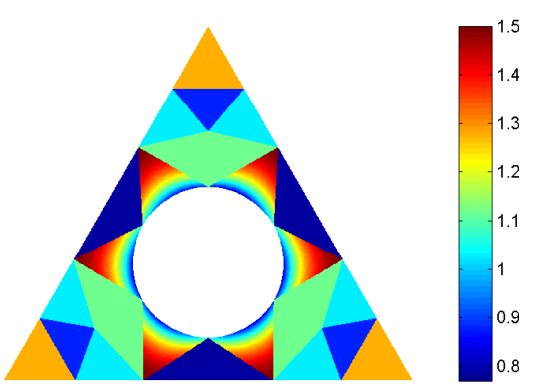

(n) Jacobian Determinant of mesh in Figure 5(i).

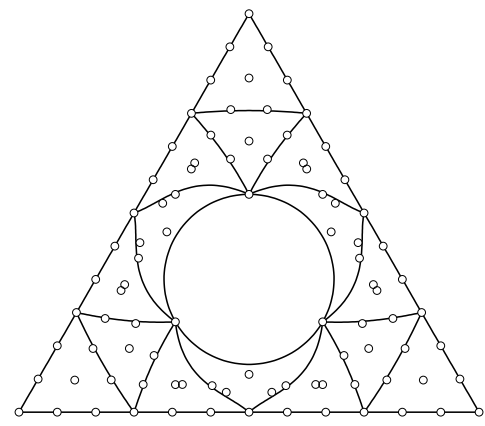

(o) Physical mesh obtained by mesh moving of Figure 5(e)

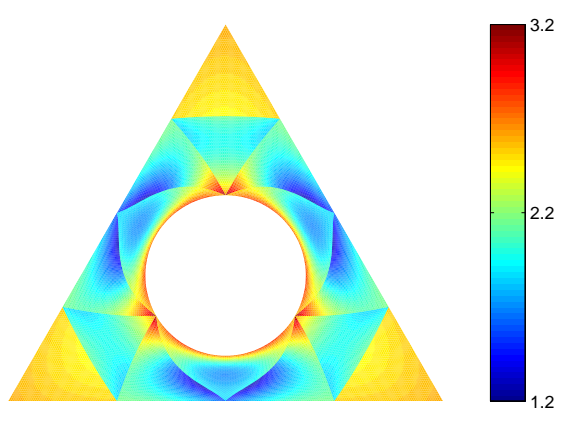

(p) Jacobian determinant of mesh in Figure 5(o)

Figure 5: Construction of parametric and physical mesh $\widehat{\Omega}_{\widehat{T}}$ and $\Omega_{T}$ (Figure $5(\mathrm{~h})$ and $5(\mathrm{i})$ respectively) for a given input domain bounded by NURBS curves (Figure 5(a). White and red squares are the end and interior control points respectively of the boundaries. White circles are the domain and control points of the mesh. 
The construction of such explicit MDS and hence the basis of the underlying spline space is not a trivial task. One approach is direct construction through macro-elements. That is, according to the connectivity of the triangle elements in a specific triangulation, one can directly choose a set of free domain points based on which all other domain points are determined through necessary continuity constraints. In this paper we refer this as the direct construction (DC) method and the resulting spaces as macro-element spaces. Some examples have been given in [33], including quintic $C^{1}$ polynomial macro-element space $\mathcal{S}_{5}^{1,2}(\widehat{T})$, quadratic $C^{1} \mathrm{PS}$ macro-element space $\mathcal{S}_{2}^{1}\left(\widehat{T}_{p s}\right)$, cubic $C^{1}$ CT macro-element space $\mathcal{S}_{3}^{1}\left(\widehat{T}_{c t}\right)$ and quintic $C^{2}$ PS macro-element space $\mathcal{S}_{5}^{2,3}\left(\widehat{T}_{p s}\right)$, where $\mathcal{S}_{5}^{1,2}(\widehat{T})$ and $\mathcal{S}_{5}^{2,3}\left(\widehat{T}_{p s}\right)$ are in fact superspline spaces with $\mathcal{S}_{5}^{1,2}(\widehat{T})$ having $C^{2}$ super smoothness at every vertex and $\mathcal{S}_{5}^{2,3}\left(\widehat{T}_{p s}\right)$ having $C^{3}$ super smoothness at every vertex and splitting point of the macro-elements and across the three interior edges not connecting to the vertices of each macro-element.

Alternatively the MDS can also be constructed by analysing a homogeneous linear system of the smoothness conditions (9) for all pairs of triangles sharing an interior edge, which is

$$
\mathrm{Ab}_{\mathcal{D}_{d, \widehat{T}}}=\mathbf{0}
$$

where $\mathbf{A}$ is a coefficient matrix depending on the geometry of the domain triangles and $\mathbf{b}_{\mathcal{D}_{d, \widehat{T}}}$ are $n$ Bézier ordinates for the domain points in $\mathcal{D}_{d, \widehat{T}}$. The dimension of the space $\mathcal{S}_{d}^{r}(\widehat{T})$ thus is

$$
\operatorname{dim} \mathcal{S}_{d}^{r}(\widehat{T})=\operatorname{dim} \mathcal{S}_{d}^{0}(\widehat{T})-\operatorname{rank} \mathbf{A} .
$$

Revealing the rank of A requires Gaussian elimination (GE). However, this is challenging in floating point arithmetic for geometry with degeneracies, which would lead to increased rank deficiencies of $\mathbf{A}$ and can be easily obscured by inexact computations [47. To overcome such issue a modified Gaussian elimination procedure based on residual arithmetic is presented in [47]. This method makes use of the fact that the vertices of the triangulation are pixels and the coordinates of the pixels are integers. Interested readers may see [4] for a more detailed discussion of this method.

In our work besides the DC method described in [33, we also use the standard GE with complete pivoting to construct the MDS. We use GE in two types of situations. The first is for triangulations constructed through macro-elements, the GE method can be used to identify stable basis for analysis. Although it is not based on residual arithmetic, it works well for the examples shown in Section 7 . The second is for general triangulations where the goal is simply to obtain smooth pre-refinement geometric map (See section 6), rather than a stable basis. 
With the known MDS, after some manipulations on matrix A, Eq.(15) can be transformed to the form

$$
\mathbf{b}_{\mathcal{D}_{d, \widehat{T}}}=\mathbf{C}^{T} \mathbf{b}_{\mathcal{M}_{d, \widehat{T}}}
$$

where $\mathbf{C}$ is called the continuity matrix. For the convenience of applying Dirichlet boundary conditions, a boundary MDS is also enforced as stated in [38], which means the complete boundary will be uniquely determined by the free nodes on the boundaries only. The rationality behind boundary MDS is as follows. The number of free nodes along the boundary equals to the total number of boundary nodes minus the number of continuity constraints along the boundary. A $C^{r}$ vertex along a boundary leads to $r$ boundary nodes to be dependent on adjacent boundary nodes. That is, among $2 r+1$ neighboring nodes of a $C^{r}$ boundary vertex, any $r$ nodes can be set as dependent nodes whose values are determined by the other $r+1$ free nodes. If all free nodes with influence on the boundary dependent nodes are on the boundary, the set of all such free nodes form a boundary MDS. In practice this formation process is accomplished by exchanging any dependent boundary node that depends on internal free nodes with one internal free node and manipulating the continuity matrix $\mathbf{C}$ accordingly. More specifically, if any boundary node $\mathbf{p}_{b}$ is dependent on at least one internal free node $\mathbf{p}_{i}$, we then re-write the smoothness constraint equation by making $\mathbf{p}_{i}$ the dependent node depending on $\mathbf{p}_{b}$. Meanwhile, the continuity matrix $\mathbf{C}$ needs to be modified accordingly as follows. A free node appears in $\mathbf{C}$ as a column with a single 1 that is otherwise all zeros. If a dependent boundary node is dependent on a free internal node, then by scaling this free basis row, and adding multiples of it to zero the boundary node's column, we replace the internal free node by one on the boundary. More details with figure illustration can be found in [38].

Combining Eq.117) and (13), the $C^{r}$ continuous function $f$ now can be expressed in terms of the free nodal ordinates $\mathbf{b}_{\mathcal{M}_{d, \widehat{T}}}$ as

$$
f(\boldsymbol{\xi})=\mathbf{b}_{\mathcal{D}_{d, \widehat{T}}^{T}}^{T} \boldsymbol{\phi}(\boldsymbol{\xi})=\mathbf{b}_{\mathcal{M}_{d, \widehat{T}}^{T}}^{T} \mathbf{C}(\boldsymbol{\xi})=\mathbf{b}_{\mathcal{M}_{d, \widehat{T}}^{T}} \boldsymbol{\psi}(\boldsymbol{\xi})
$$

where

$$
\psi(\xi)=\mathbf{C} \phi(\xi)
$$

is a set of global $C^{r}$ basis functions composed as the linear combinations of the $C^{0}$ Bernstein basis $\phi(\xi)$. In this way, we can represent the $C^{r}$ Bézier elements in terms of the $C^{0}$ elements. A similar procedure known as Bézier extraction has been explained in data fitting and the FEM [20], and later used in IGA with NURBS [48] and T-spline [49] elements respectively.

\subsection{Construction of $C^{r}$ geometric map $\mathbf{G}(\boldsymbol{\xi})$}

After identifying the free control points $\mathbf{p}_{i}^{f}$ corresponding to the free domain points in $\mathcal{M}_{d, \widehat{T}}$, all the control points $\mathbf{p}$ for rTBS elements in the physical mesh $T$ are overridden with a set of control points satisfying the $C^{r}$ continuity constraints

$$
\mathbf{p}=\mathbf{C}^{T} \mathbf{p}^{f}
$$

Note that generally the boundary control points will not be overridden since the boundary is already $C^{r}$ in the previous $C^{0}$ parametrization (Step (3) in Section 3.1), unless some $C^{q}(0 \leq q<r)$ knot points are mapped to corners of the parametric domain which invokes singularities in the parametrization [32. An example is given in Figure 6 where a $C^{1}$ physical mesh is constructed by using CT macro-elements from the $C^{0}$ mesh 
in Figure 5(o), In the $C^{1}$ physical mesh in Figure 6(a), there are three singularities which are mapped to the three corner points of the inner triangle in Figure 6(b). The control points in the boundary MDS determining the inner circle are indicated with larger marker size. As can be seen, the Jacobian determinants at the three singularities are zero, since the control points around these singularities are colinear (Figure 6(a)), meaning the $C^{1}$ smoothness is maintained. In case of critical analysis results are desired around such potential singularities, one can alleviate their influence by using optimization technique such as [32], or by locally reducing the continuity around them to $C^{0}$, as explained in [38]. In addition, for complex geometries it may invoke distortion or even foldings in the $C^{r}$ geometric map when overriding the interior control points. When this happens, a proper optimization technique such as 32] can be used.

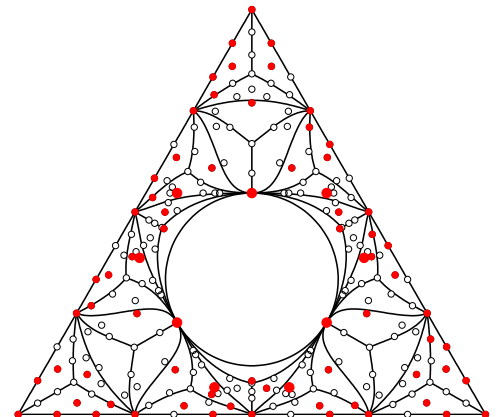

(a) $C^{1}$ physical mesh constructed by using CT macroelements from the $C^{0}$ mesh in Figure $5(\mathrm{o})$ Points in the boundary MDS of the inner circle are indicated with larger marker size.

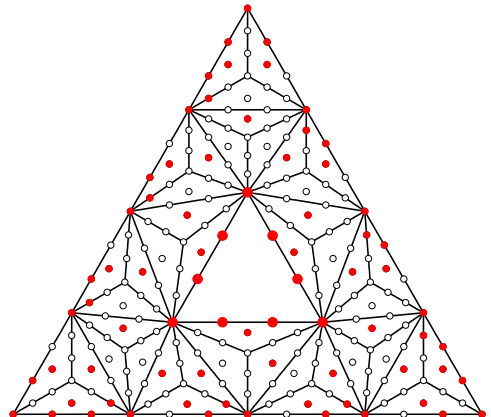

(b) Corresponding $C^{1}$ parametric mesh. Points in the boundary MDS of the inner circle are indicated with larger marker size.

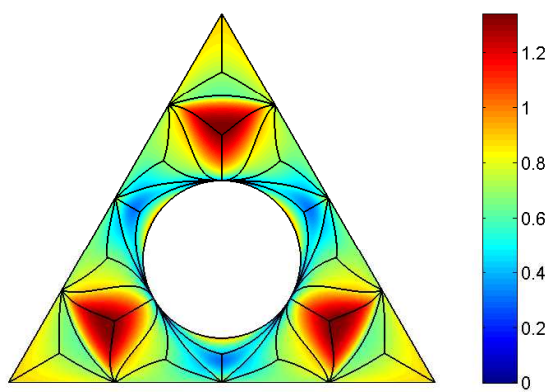

(c) Jacobian determinant of mesh in Figure 6(a), The Jacobian determinants at the three singular points are zero.

Figure 6: $C^{1}$ mesh constructed by using CT macro-elements and its Jacobian determinant. In the $C^{1}$ physical mesh in Figure 6(a) there are three singular points which are mapped to the three corner points of the inner triangle in Figure 6(b). The control points in the boundary MDS determining the inner circle are indicated with larger marker size. The Jacobian determinants at the three singular points are zero, since the control points around these singularities are colinear (Figure 6(a)), meaning the $C^{1}$ smoothness is maintained.

Now we obtain the $C^{r}$ geometric map $\mathbf{G}(\boldsymbol{\xi}): \widehat{\Omega} \rightarrow \Omega$ in terms of rational $C^{r}$ basis functions $\psi_{i}(\boldsymbol{\xi})$, or equivalently, the rational $C^{0}$ Bernstein basis functions $\phi_{j}(\boldsymbol{\xi})$ as

$$
\mathbf{G}(\boldsymbol{\xi})=\sum_{i}^{m} \mathbf{p}_{i}^{f} \psi_{i}(\boldsymbol{\xi})=\sum_{j}^{n} \mathbf{p}_{j} \phi_{j}(\boldsymbol{\xi}),
$$

where $m$ and $n$ are the dimension of the space $\mathcal{S}_{d}^{r}$ and $\mathcal{S}_{d}^{0}$ respectively.

\section{Isogeometric analysis using rTBS elements}

In this section we describe the method of isogeometric analysis using rTBS elements where the classical Galerkin formulation is applied. The problems considered in this paper include linear elasticity and Poisson problem. The governing equation for the 
linear elasticity is

$$
\begin{cases}\nabla \cdot \boldsymbol{\sigma}+\boldsymbol{b}=\mathbf{0} & \text { on } \Omega \\ \boldsymbol{\sigma}=\mathbf{D} \nabla_{s} \mathbf{u} & \\ \boldsymbol{\sigma} \cdot \boldsymbol{n}=\boldsymbol{t} & \text { on } \Gamma_{t} \\ u=\bar{u} & \text { on } \Gamma_{u}\end{cases}
$$

where $\mathbf{D}$ is the elasticity matrix, $\mathbf{b}$ and $\boldsymbol{t}$ refer to body force and traction respectively, $\mathbf{u}$ is the displacement, $\Gamma_{t}$ and $\Gamma_{u}$ are the portions of the boundary where traction and displacement are specified respectively. The Poisson problem is defined as

$$
\begin{cases}-\nabla^{2} u=f & \text { in } \Omega, \\ u=\bar{u} & \text { on } \Gamma,\end{cases}
$$

where $f: \Omega \rightarrow \mathbb{R}$ is a given function and $\bar{u}$ denotes prescribed boundary values.

Using the basis constructed in the previous section, we approximate the solution in the corresponding parametric domain as

$$
\hat{u}(\boldsymbol{\xi})=\sum_{i} u_{i} \psi_{i}(\boldsymbol{\xi})=\boldsymbol{u}^{T} \boldsymbol{\psi}
$$

where $u_{i}$ corresponds to the approximate solution's Bézier ordinate at the $i$-th domain point in the parametric domain $\widehat{\Omega}_{\widehat{T}}$, as illustrated in Figure 1 . The solution $u(\boldsymbol{x})$ over the domain $\Omega_{T}$ in the physical space is obtained by composing $\hat{u}(\boldsymbol{\xi})$ with the inverse of the geometric mapping $\mathbf{G}^{-1}$ such that $u(\boldsymbol{x}): \Omega \mapsto \mathbb{R}^{2}$,

$$
u(\boldsymbol{x})=\hat{u}(\boldsymbol{\xi}) \circ \mathbf{G}^{-1}(\boldsymbol{x}) .
$$

After inserting the approximate solution and basis functions into the corresponding weak form of the PDE, we obtain the following mass and stiffness matrices respectively as

$$
\begin{gathered}
\mathbf{M}_{0}=\int_{\Omega} \boldsymbol{\phi} \cdot \boldsymbol{\phi} d \Omega, \\
\mathbf{K}_{0}=\int_{\Omega} \nabla \boldsymbol{\phi} \cdot \nabla \boldsymbol{\phi} d \Omega,
\end{gathered}
$$

for $C^{0}$ elements. For $C^{r}$ elements, we calculate the mass and stiffness matrices using the fact that the $C^{r}$ basis $\boldsymbol{\psi}$ are linear combinations of $C^{0}$ basis $\phi$,

$$
\begin{gathered}
\mathbf{M}_{r}=\int_{\Omega} \boldsymbol{\psi} \cdot \boldsymbol{\psi} d \Omega=\int_{\Omega}(\boldsymbol{C} \boldsymbol{\phi}) \cdot(\boldsymbol{C} \boldsymbol{\phi}) d \Omega=\boldsymbol{C}^{T} \widetilde{\mathbf{M}}_{0} \boldsymbol{C} \\
\mathbf{K}_{r}=\int_{\Omega} \nabla \boldsymbol{\psi} \cdot \nabla \boldsymbol{\psi} d \Omega=\int_{\Omega}(\boldsymbol{C} \nabla \boldsymbol{\phi}) \cdot(\boldsymbol{C} \nabla \boldsymbol{\phi}) d \Omega=\boldsymbol{C}^{T} \widetilde{\mathbf{K}}_{0} \boldsymbol{C},
\end{gathered}
$$

where $\widetilde{\mathbf{M}}_{0}$ and $\widetilde{\mathbf{K}}_{0}$ are the mass and stiffness matrices respectively for the same $C^{r}$ elements in terms of the $C^{0}$ basis $\phi$. The difference between $\widetilde{\mathbf{M}}_{0}$ and $\mathbf{M}_{0}, \widetilde{\mathbf{K}}_{0}$ and $\mathbf{K}_{0}$ is due to the potential relocation of the control points to satisfy the $C^{r}$ continuity constraints for the $C^{r}$ elements. The assembly process for such matrices is different from the one in [38], where the entries in $\mathbf{M}_{r}$ and $\mathbf{K}_{r}$ are calculated directly after identifying the basis function $\psi_{i}$ supporting each element from the continuity matrix $\mathbf{C}$. Instead we 
assemble $\widetilde{\mathbf{M}}_{0}$ and $\widetilde{\mathbf{K}}_{0}$ first using the $C^{0}$ basis $\phi$ as in classical $C^{0}$ FEM and then multiply the continuity matrix $\mathbf{C}$ to obtain $\mathbf{M}_{r}$ and $\mathbf{K}_{r}$ as shown in Eq. (28) and (29). This implementation can be readily applied in any existing FEM routine without changing the assembly process. The numerical integration is performed in each element (microelement if split is used) by using standard and collapsed [50] Gaussian quadrature rules on the boundaries and element interiors respectively. Specifically, the integrals are first pulled back onto the parametric domain and then onto a parent element of right-angled isosceles triangle, as shown in Figure 1.

Due to the use of boundary MDS mentioned earlier, the Dirichlet boundary conditions can be imposed similarly as in NURBS based IGA. Typical strategies include the least square method (used in this work), weak imposition using Lagrange multiplier and an improved transformation method [51].

\section{Approximation property of the rTBS space}

It is already known that the set of Bernstein basis functions of degree $d$ over a triangulation form a basis for the polynomial space of degree $d[33]$, thus for $C^{0}$ basis the same error estimate holds as for classical finite element methods and optimal convergence rates can be guaranteed. In this section we focus on how well $C^{r}$ continuous functions can be approximated by rTBS.

It has been proven in [33] that if there exists a subspace of $\mathcal{S}_{d}^{r}(\widehat{T})$ with a stable local minimal determining set, then $\mathcal{S}_{d}^{r}(\widehat{T})$ has the approximation power up to $d+1$. That is for every $f \in H^{d+1}$, there exists a spline $s \in \mathcal{S}_{d}^{r}(\widehat{T})$ such that

$$
|f-s|_{W^{k, d+1}(\widehat{\Omega})} \leq C h_{\widehat{T}}^{d+1-k}|f|_{W^{d+1, d+1}(\widehat{\Omega})}, \quad 0 \leq k \leq d,
$$

where $H^{k}$ and $W^{k, p}$ are the Hilbert and Sobolev spaces respectively with $|\cdot|$ the associated seminorm, $h_{\widehat{T}}$ is the length of the longest edge in $\widehat{T}$, the constant $C$ depends only on $d$ and the smallest angle in $\widehat{T}$, and the Lipschitz constant of the boundary of $\widehat{\Omega}$.

Although the result (30) is derived in the TBS space, following NURBS based IGA in [39, 52], a similar result in the rTBS space can be derived as

$$
\left|f-\Pi_{\mathcal{S}} f\right|_{H^{k}(\tau)} \leq C_{w} h_{\widehat{T}}^{d+1-k}|f|_{W^{d+1, d+1}(\widehat{\Omega})}, \quad \forall f \in H^{d+1}, 0 \leq k \leq d,
$$

where $\Pi_{\mathcal{S}}$ is the projector on the rTBS space $\mathcal{S}_{d}^{r}$, the constant $C_{w}$ differs from $C$ by the extra dependence on the weight function $w$ and $\tau$ is an element in the triangulation $\widehat{T}$. Finally we define the projector $\Pi_{\mathcal{U}}: L^{2}(\Omega) \rightarrow \mathcal{U}_{d}^{r}$ as

$$
\Pi_{\mathcal{U}} f:=\left(\Pi_{\mathcal{S}}(f \circ \mathbf{G})\right) \circ \mathbf{G}^{-1}, \quad \forall f \in L^{2}(\Omega),
$$

where $\mathcal{U}_{d}^{r}$ is the space of rTBS on the physical domain $\Omega$ (the push-forward of the rTBS space $\mathcal{S}_{d}^{r}$ on the parametric domain $\left.\widehat{\Omega}\right)$, as shown in Figure 7 .

Now the error estimate on the physical domain $\Omega$ can be derived as

$$
\left|f-\Pi_{\mathcal{U}} f\right|_{H^{k}(T)} \leq C_{w} h_{T}^{d+1-k} \sum_{i=0}^{d+1}\|\nabla \mathbf{G}\|_{L^{\infty}\left(\mathbf{G}^{-1}(T)\right)}^{i-d-1}|f|_{H^{i}(T)}, \quad \forall f \in H^{d+1}(\Omega),
$$

where $\mathbf{G}$ is the geometric map, $T=\mathbf{G}(\widehat{T})$, and $h_{T}$ is the longest element edge in $T$. Eq.(33) implies the rTBS space on the physical domain delivers the optimal rate of convergence, which is $d+1-k$ in terms of the error norm in $H^{k}$ space for polynomials of degree $d$, provided that there is a set of local stable basis for $\mathcal{S}_{d}^{r}$ and the geometric map $\mathrm{G}$ remains the same for different mesh size $h_{T}$. 


\section{Pre-refinement smooth geometric map}

As shown in the previous section, in order to evaluate the convergence rate upon $h$-refinement, the geometric map must remain the same during refinement. In the section below, we first show the need for a smooth pre-refinement geometric map. We then introduce a strategy to construct a pre-refinement geometric map that possesses sufficient smoothness to maintain the consistency of the geometric map for all subsequent refinements.

\subsection{Need for a pre-refinement smooth geometric map}

Let $\triangle_{0} \subset \triangle_{1} \subset \triangle_{2} \subset \cdots \subset \triangle_{n}$ be a nested sequence of triangulations where $\triangle_{k-1} \subset \triangle_{k}$ means $\triangle_{k}$ is a refinement of $\triangle_{k-1}$ by subdividing each triangle in $\triangle_{k-1}$ into several sub-triangles. We denote the $C^{r}$ spline space defined on $\triangle_{k}$ as $\mathcal{S}_{d}^{r}\left(\triangle_{k}\right)$. If the mesh sequences in the parametric domain are nested, that is, $\widehat{T}_{0} \subset \widehat{T}_{1} \subset \widehat{T}_{2} \subset \cdots \subset \widehat{T}_{n}$, and the resulting triangulations in the physical domain under the geometric map $\mathbf{G}_{k}: \widehat{T}_{k} \mapsto T_{k}$ are also nested as $T_{0} \subset T_{1} \subset T_{2} \subset \cdots \subset T_{n}$, we then say the geometric map $\mathbf{G}_{k}$ for the space $\mathcal{S}_{d}^{r}\left(\triangle_{k}\right)$ is consistent during the refinement sequence.

For accurate isogeometric analysis with $C^{r}$ rTBS elements, elements need to be sufficiently small. A simple and intuitive way is to first obtain $C^{0}$ coarse mesh from the procedure outlined in Section 3.1, perform uniform refinement on the $C^{0}$ mesh until the elements are small enough for accurate analysis, and then impose $C^{r}$ smoothness constraints through a macro-element based DC technique or GE. That is, for space $\mathcal{S}_{d}^{r}$, we first create a nested sequence of triangulations $T_{0} \subset T_{1} \subset T_{2} \subset \cdots \subset T_{n}$ in the physical domain, and then impose $C^{r}$ continuity constraints on each mesh $T_{k}$ and relocate the dependent control points to ensure $C^{r}$ smoothness. This is the route used in our earlier paper [38. Although such a refine-then-smooth approach is able to create arbitrarily small rTBS elements with desired continuity for analysis, the resulting geometric map may not be consistent during the refinement. That is, the resulting elements are not nested after the refinement. As such, optimal convergence cannot be achieved with such an approach. We use two examples below to demonstrate such a lack of consistency in the resulting $C^{r}$ geometric map with the refine-then-smooth approach.

Our notation in various refinement and splits are as follows. We use the subscripts $u, c t, p s$ to indicate uniform refinement, CT split and PS split respectively and use them in the same sequential order as these refinements are performed, while the superscript indicates the order of smoothness. For example, $T_{c t, u, u, p s}^{1}$ represents a $C^{1}$ smooth mesh obtained by performing a CT split followed by two uniform refinements and a PS split on the mesh $T$. Note that the CT and PS split are usually followed by imposing continuity constraints. Here we use $c t^{*}$ and $p s^{*}$ to indicate the respective split before imposing continuity constraints. 


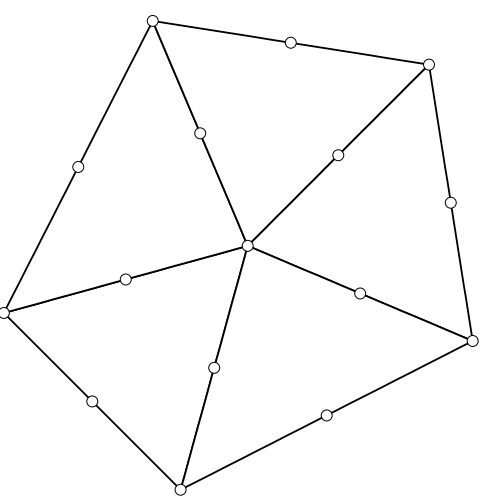

(a) Initial quadratic parametric mesh $\widehat{T}^{0}$.

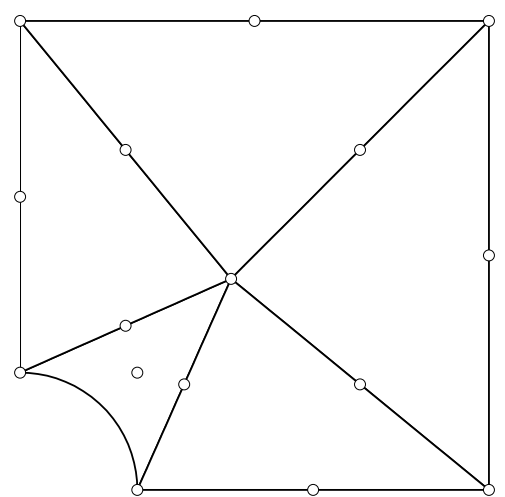

(b) Initial quadratic physical mesh $T^{0}$.

Figure 8: Initial parametric and $C^{0}$ physical mesh of a domain with curved boundary.

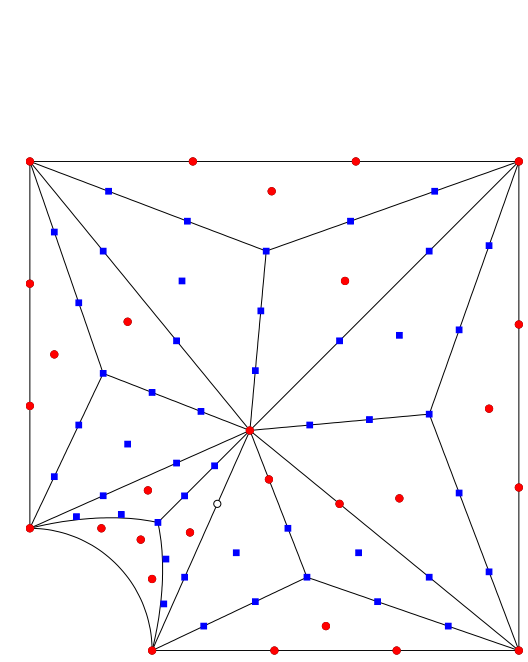

(a) $T_{c t *}^{0}$.

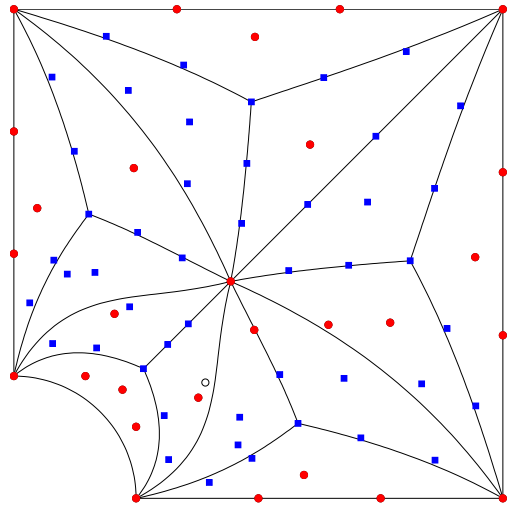

(d) $T_{c t}^{1}$.

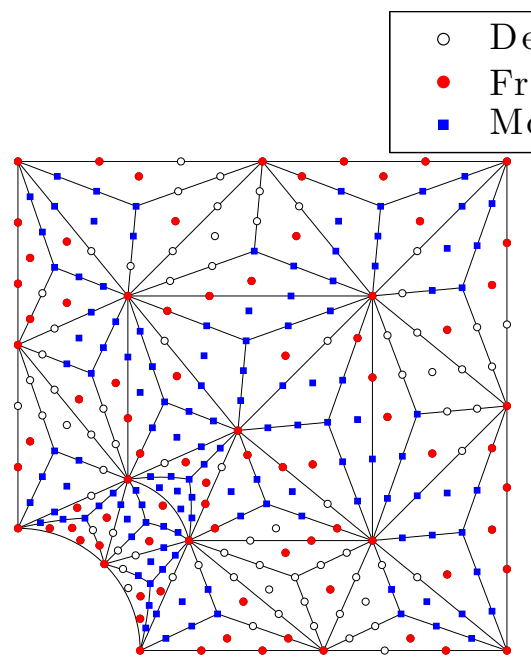

(b) $T_{u, c t *}^{0}$.

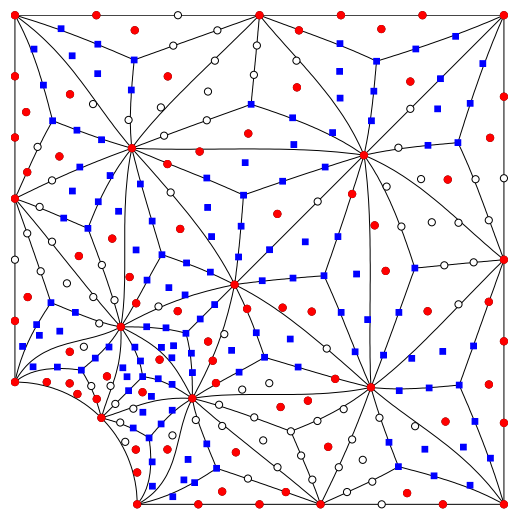

(e) $T_{u, c t}^{1}$.
Dependent nodes

Free nodes

Moved dependent nodes

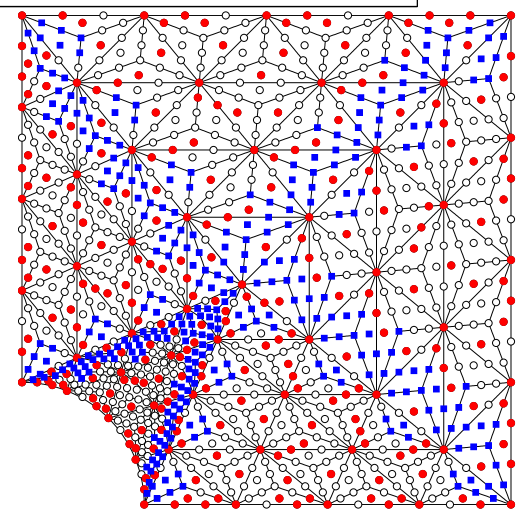

(c) $T_{u, u, c t *}^{0}$.

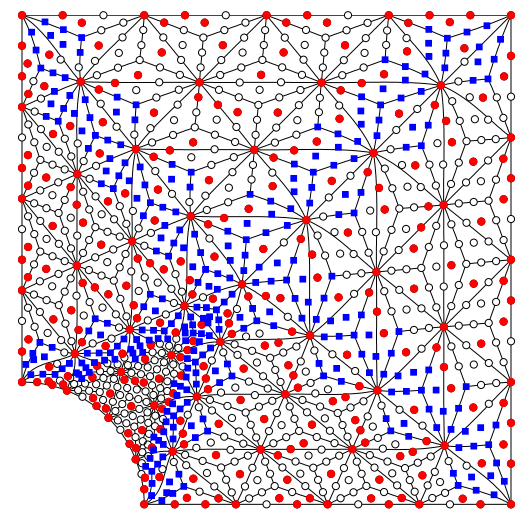

(f) $T_{u, u, c t}^{1}$.

Figure 9: Uniform refinement of $C^{0}$ elements in Fig. 8, followed by the CT split to obtain $C^{1}$ smoothness, leads to inconsistency in the resulting geometric map. The first row are $C^{0}$ meshes where red points are free points, white circles and blue squares are all dependent control points except blue squares are dependent points which do not satisfy the continuity condition while the white circles do. That is, the blue square control points in top row are relocated to their different respective positions in the bottom row to satisfy the continuity constraints. 
An example is given below where a domain is initially parametrized into five $C^{0}$ elements, as shown in Figure 8. Figure 9 shows a nested sequence of triangulations from such initial $C^{0}$ elements through uniform refinement. CT splits are then performed to obtain splines in the $\mathcal{S}_{3}^{1}$ space. The first row are $C^{0}$ meshes before continuity constraints are actually imposed, where the red points are free control points whose locations can be chosen freely, the white circles and blue squares are dependent control points whose locations are determined through the continuity constraints. Particularly, the white circles have already satisfied the continuity condition, while the blue squares are not and need to be relocated to obtain $C^{1}$ smoothness. As can be seen, the blue squares are distributed only near the common edges shared by the initial five elements in Figure 8(b). After relocating the control points, as shown in the bottom row, the meshes have been locally changed and are no longer nested. The relocation of some control points $\mathbf{p}_{i}$ (blue squares) to satisfy the continuity constraints thus leads to a change of the geometric map according to Eq.(21).

Our second example concerns a kind of macro-elements in the superspline space $\mathcal{S}_{d}^{r, \rho}$, $\rho>r$, as defined in (11), where supersmoothness $C^{\rho}$ happens at the vertices or edges of the macro-triangles. Uniform refinement of such elements followed by the same macroelement technique to achieve supersmoothness at macro-element vertices or edges would lead to inconsistent geometric maps. An example is given in Figure 10 where the initial mesh (Figure 10a) in superspline space $\mathcal{S}_{5}^{1,2}$ is obtained by DC based on the quintic $C^{1}$ macro-element technique [33]. This initial mesh is globally $C^{1}$ but with $C^{2}$ smoothness at the vertices of macro-triangles. If we start refining from this initial $T^{1,2}$ mesh as shown in Figure 10(a) where the smoothness at the green stars are $C^{2}$ and the smoothness at the grey stars in the refined mesh is still $C^{1}$. In order to obtain a stable basis in the $\mathcal{S}_{5}^{1,2}$ with the same macro-element technique, all macro vertices need to be $C^{2}$ smooth. Thus we need to relocate the control points marked by the blue squares to achieve $C^{2}$ smoothness at all macro vertices since many of them only possess $C^{1}$ smoothness before refinement. As the mesh is refined, there are more and more vertices that need to be relocated to achieve $C^{2}$ smoothness. Such relocation of control points to achieve higherorder smoothness at more and more vertices and edges of refined macro-triangles, due to supersmoothness requirement at vertices of macro-triangles in the $\mathcal{S}_{5}^{1,2}$ macro-element space, leads to the loss of consistency of the geometric map.

To overcome such inconsistency of the geometric map during the refinement, we recommend a geometric map with sufficient smoothness be constructed before the refinement. For the usual $C^{r}$ splines, the pre-refinement geometric map should be at least $C^{r}$ smooth. For superspline space $\mathcal{S}_{d}^{r, \rho}$ where supersmoothness occurs at the vertices or edges of macro-triangles, we recommend to construct a $C^{\rho}$ pre-refinement map based on which we perform refinement. In this way, all refined elements are globally $C^{\rho}$ smooth and the supersmoothness required at those vertices and edges are therefore satisfied. The refinement sequence is nested and the geometric map remains unchanged.

Note if supersmoothness happens at the interior vertices or edges of macro-triangles after the splits, refinement of such elements does not need to relocate dependent control points to satisfy the continuity constraints. This is because, at these internal vertices and edges of a macro-triangle, the continuity is already $C^{\infty}$. For example, although the quadratic $C^{1}$ PS macro-element and cubic $C^{1} \mathrm{CT}$ macro-element spaces are also superspline spaces, their supersmoothness occurs inside the macro-elements where the geometric smoothness is infinity. Thus they can be treated as regular $C^{r}$ spaces in terms of smoothness requirement of the pre-refinement geometric map. 


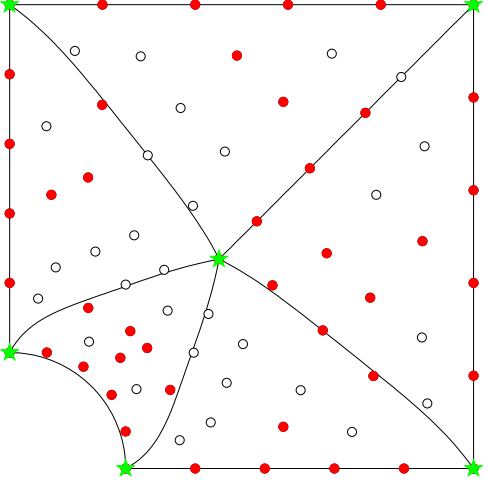

(a) $T^{1,2}$ (initial mesh).

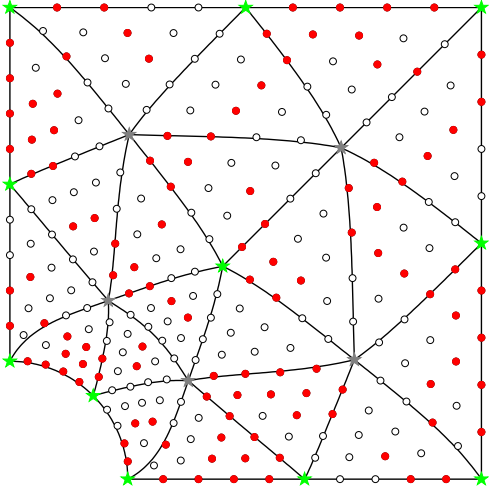

(b) $T_{u}^{1,2}$ (one refinement).

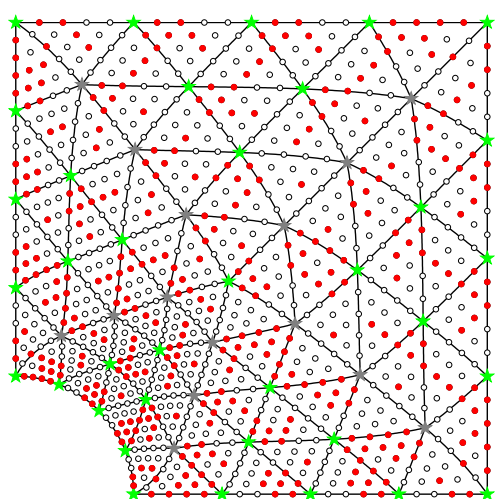

(c) $T_{u, u}^{1,2}$ (two refinements).

Figure 10: Uniform refinement of $C^{1,2}$ elements in superspline space $\mathcal{S}_{5}^{1,2}$, then with the quintic $C^{1}$ macro-element technique to obtain the global $C^{1}$ smoothness with $C^{2}$ smoothness at vertices of all macrotriangles, leads to inconsistent geometric map. Red points are free points, white circles are dependent control points which satisfy the needed smoothness condition and blue squares are dependent control points which do not satisfy the smoothness condition.

\subsection{Construction of a pre-refinement smooth geometric map}

Our remedy to avoid the inconsistency of the $C^{r}$ geometric map during the refinement is to construct a pre-refinement map that is sufficiently smooth. We then apply refinements on it, and follow up with the $C^{r}$ continuity constraints to obtain a stable basis for $C^{r}$ rTBS elements, as outlined in Section 3.2. The refined mesh inherits the continuity. The refined control points therefore do not need to be relocated since they already satisfy the continuity conditions. Thus the resulting meshes are $C^{r}$ smooth and nested, and the geometric map remains the same for all subsequent refinements.

By the previous sections it is straightforward to obtain such a sufficiently smooth pre-refinement mesh for a given domain. If the domain is bounded by straight line segments, recalling that the domain points in the parametric mesh always satisfy the smoothness condition, we can generate the control points in the location obtained by an affine transformation of the domain points in the parametric mesh, then the physical mesh obtained will satisfy the needed smoothness condition as well. If the domain has curved boundaries, we can either use the method in Section 3.2 which constructs a $C^{r}$ mesh with a set of stable local basis, or simply relocate the control points to satisfy the smooth conditions by Gaussian elimination.

Two examples are given for the mesh refinement sequences in $\mathcal{S}_{3}^{1}\left(T_{c t}\right)$ and $\mathcal{S}_{5}^{1,2}(T)$, as shown in Figure 11 where the meshes in a refinement sequence are overlaid. The first row shows that if no smooth pre-refinement map is constructed, the geometric map clearly changes during refinement. Particularly, for the mesh sequence in $\mathcal{S}_{5}^{1,2}(T)$, even if the movements of the blue square points are so small, it will affect the convergence rates, as will be shown later in the numerical examples. In the second row, we first construct a pre-refinement smooth geometric map that is $C^{1}$ for space $\mathcal{S}_{3}^{1}\left(T_{c t}\right)$ and $C^{2}$ for $\mathcal{S}_{5}^{1,2}(T)$ by relocating the control points using Gaussian elimination, then refine the meshes based on the smooth map. It can be seen that the map remains the same during the refinement.

It is worthy to mention the difference between the pre-refinement $C^{r}$ smooth geometric map in this section and the $C^{r}$ map in Section 3.2. The reason for a pre-refinement smooth geometric map is purely geometric. That is, we just need to create a $C^{r}$ map which maps the parametric domain to the physical domain and recovers the original boundary, without 


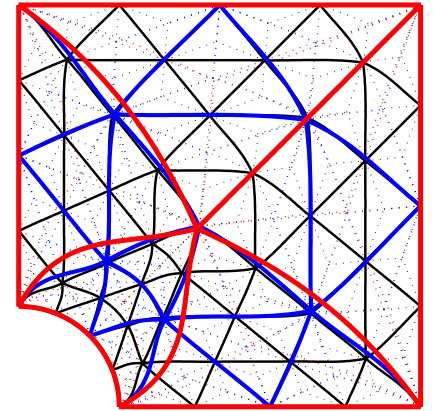

(a) Overlapped mesh sequence in $\mathcal{S}_{3}^{1}\left(T_{c t}\right)$ without pre-refinement smooth map.

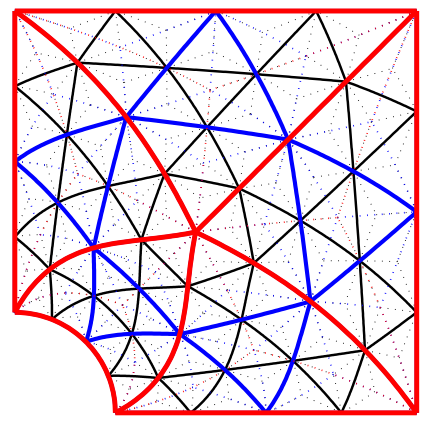

(d) Overlapped mesh sequence in $\mathcal{S}_{3}^{1}\left(T_{c t}\right)$ with prerefinement smooth map.

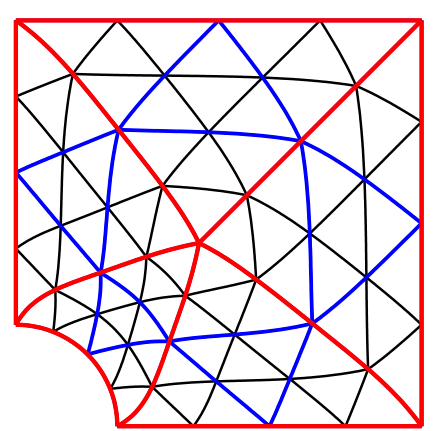

(b) Overlapped mesh sequence in $\mathcal{S}_{5}^{1,2}(T)$ without pre-refinement smooth map. The invisible difference among overlapped meshes is shown in Figure 11(c).

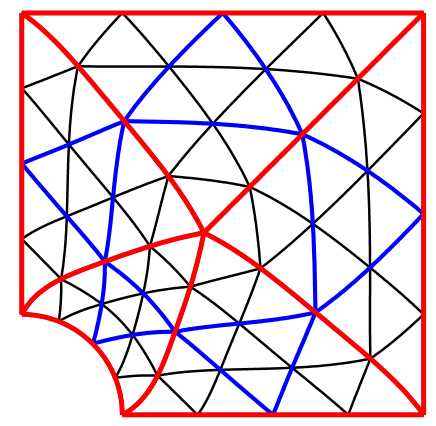

(e) Overlapped mesh sequence in $\mathcal{S}_{5}^{1,2}(T)$ with prerefinement smooth map.

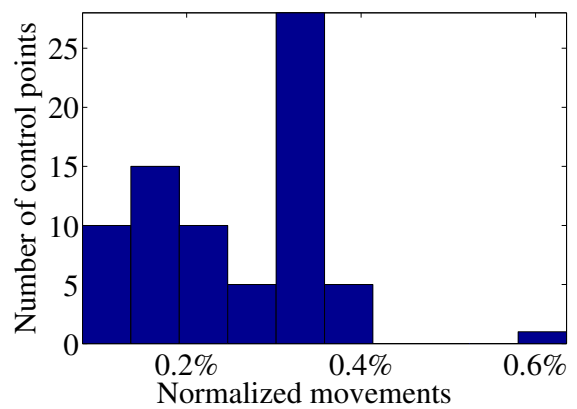

(c) Normalized movements (with respect to the maximum element size) of control points marked with blue squares in $T_{u, u}^{1,2}$ (Figure $10(\mathrm{c})$ to satisy the continuity constraints.

Figure 11: Comparison of geometric map during $h$-refinement (first row) without and with pre-refinement (second row) smooth geometric map in $\mathcal{S}_{3}^{1}\left(T_{c t}\right)$ and $\mathcal{S}_{5}^{1,2}(T)$. The meshes in a refinement sequence are overlapped with blue, black and red color representing mesh with no $h$-refinement, after $1 h$-refinement and $2 h$-refinement respectively. The solid lines are the edges of the macro-elements while the dashed lines are the splitting edges of the micro-elements. The first row clearly shows that the geometric map has changed during refinement without pre-refinement smooth map. The second row shows the geometric map remains the same with pre-refinement smooth geometric map. 
the need for a set of stable local basis for analysis. Such a smooth pre-refinement map is needed so that, during the refinement, the control points do not need to relocate since they would already satisfy the continuity conditions. Thus, the pre-refinement map is kept consistent during the refinement. On the other hand, in order to approximate a field in analysis a set of stable basis is necessary and should be constructed by the methods presented in Section 3.2. For example, in cubic space $\mathcal{S}_{3}^{1}\left(T_{c t}\right)$ with CT split, if we have created a $C^{1}$ pre-refinement map using CT split, as shown in Figure 12(a), even if it is $C^{1}$ after uniform refinement (Figure 12(b)), we still need to perform CT split again in order to construct a set of stable local basis in $\mathcal{S}_{3}^{1}\left(T_{c t}\right)$ to be used in analysis, as shown in Figure 12(c).

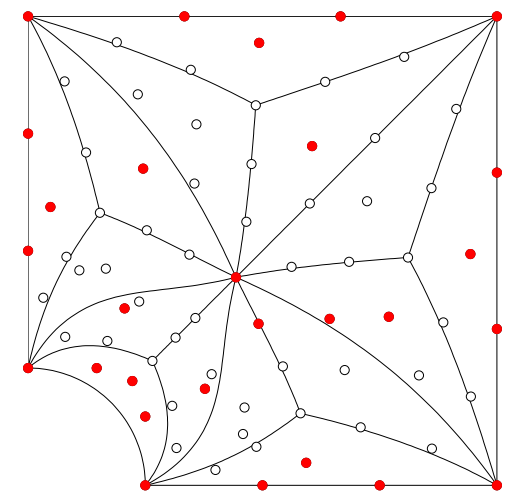

(a) Cubic $C^{1}$ mesh obtained by DC with CT macro-elements. The space $\mathcal{S}_{3}^{1}$ is well defined on a cubic mesh with CT macroelements.

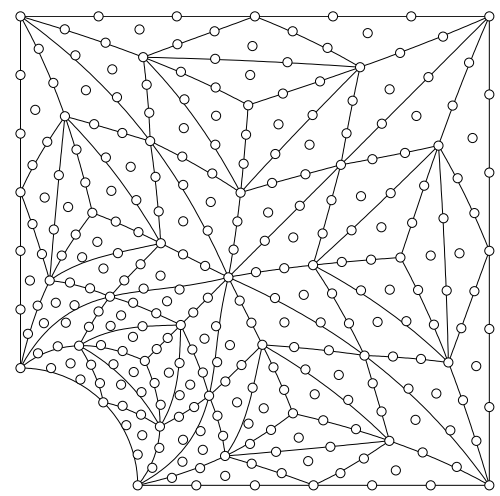

(b) $C^{1}$ mesh after uniformly refining mesh in (a). The dimension of the space $\mathcal{S}_{3}^{1}$ on this triangulation is unknown.

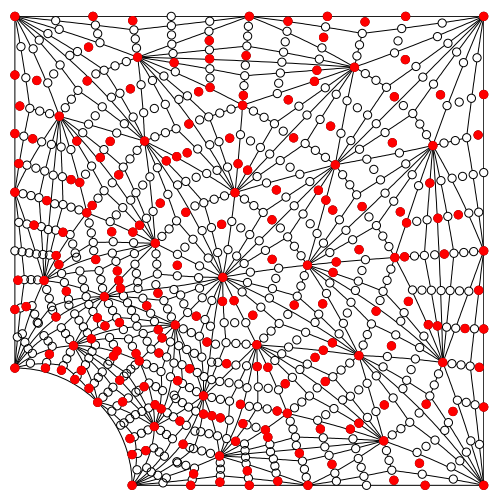

(c) CT split is perfomred again on the mesh in (b) to construct a set of stable local basis that defines $\mathcal{S}_{3}^{1}$.

Figure 12: Smooth-refine-smooth procedure. The purpose of the first CT split is to construct a prerefinement smooth geometric map, while the CT split at the last step is to construct a set of $C^{1}$ stable local basis for analysis.

\section{Numerical results}

In this section we demonstrate how optimal convergence rates can be achieved using rTBS-based isogeometric analysis for different problems with different elements. Only macro-elements are used as discussed in Section 5 to ensure optimal rates can be achieved. We first show that on a domain bounded by straight line segments, optimal convergence rates are achieved in all presented spaces. Then we demonstrate that, for domains with curved boundaries, if no pre-refinement smooth map is constructed to keep the $C^{r}$ geometric map consistent during refinement, the convergence rates are lower than optimal. Then with the pre-refinement smooth map as discussed in Section 6, optimal convergence rates are achieved in all cases. The results are demonstrated on two examples, one Poisson problem on a complex domain with three holes and one elastic problem on a plate domain. We also show the advantage of local refinement in rTBS for the elasticity problem. Note, in all examples below, the element size in the convergence study refers to the maximal length of the edges in the micro-elements.

\subsection{Domain with all straight boundaries: Poisson problem}

The first example is a triangular domain with a triangular hole as shown in Figure 13. All sides consist of straight line segments. The governing equation is 23) with the 
open set domain $\Omega$ being defined as

$$
\begin{aligned}
& \Omega:=\left\{(x, y) \mid\left[\left(0<x \leq \frac{3}{2}\right) \&(0<y<3 \sqrt{3} x) \cup\left(\frac{3}{2}<x<1\right) \&(0<y<3 \sqrt{3}(1-x))\right] \backslash\right. \\
& \quad\left[\left(\frac{9}{8} \leq x \leq \frac{3}{2}\right) \&\left(\frac{3 \sqrt{3}}{8} \leq y \leq 3 \sqrt{3}\left(x-\frac{1}{4}\right)\right) \cup\left(\frac{3}{2}<x \leq \frac{15}{8}\right)\right. \\
& \left.\left.\quad \&\left(\frac{3 \sqrt{3}}{8} \leq y \leq 3 \sqrt{3}\left(\frac{3}{4}-x\right)\right)\right]\right\} .
\end{aligned}
$$

The body force is

$$
f(x, y)=-\left(x^{2}+y^{2}\right) e^{x y},
$$

and the exact solution is given by

$$
u(x, y)=e^{x y} .
$$

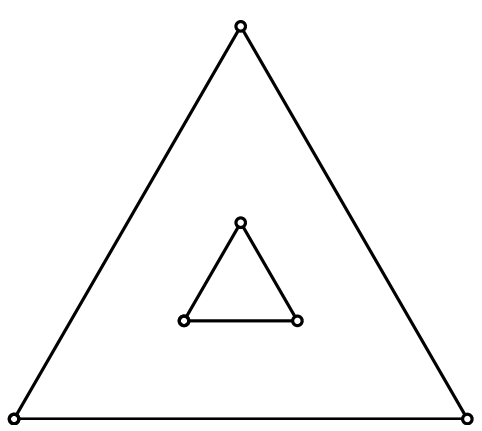

(a) Problem domain.

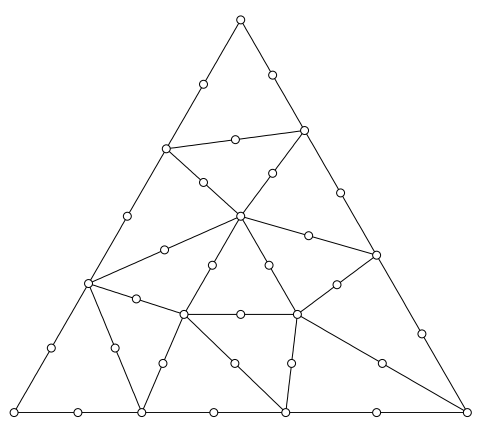

(d) Quadratic mesh $\mathcal{S}_{2}^{0}$.

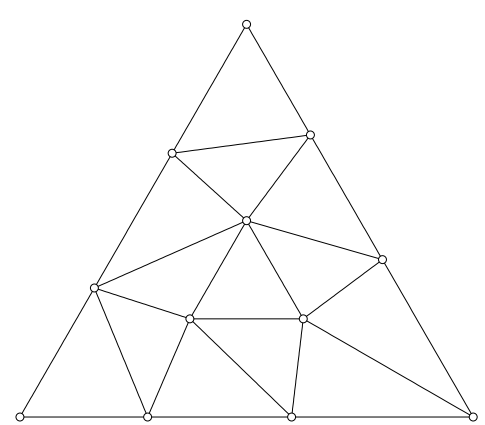

(b) Initial parametric mesh.

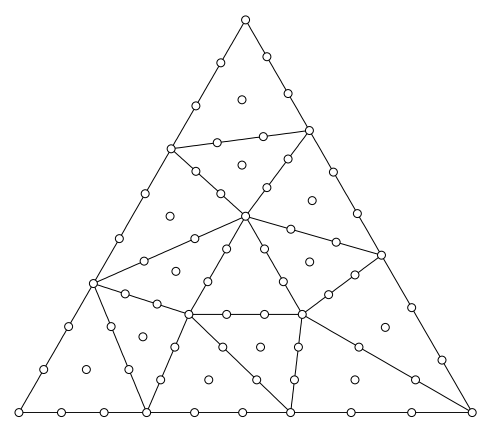

(e) Cubic mesh $\mathcal{S}_{3}^{0}$.

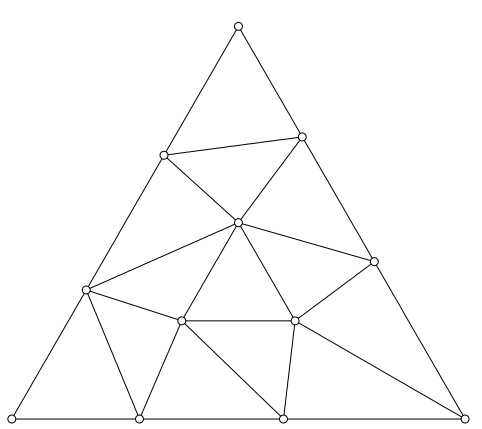

(c) Initial physical mesh.

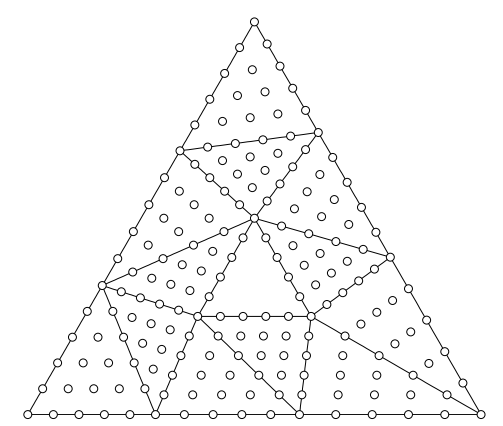

(f) Quintic mesh $\mathcal{S}_{5}^{0}$.

Figure 13: Problem domain and initial $C^{0}$ meshes for the Poisson problem.

Based on our parametrization strategy in Section 3, the parametric domain is the same as the physical domain since the boundary edges are all straight. The initial linear mesh is obtained by the Delaunay triangulation of the problem domain as shown in Figure 13. By degree elevation we obtain meshes corresponding to quadratic, cubic and quintic $C^{0}$ spline spaces respectively as shown in the second row of Figure 13 . We only show the physical meshes since the parametric meshes are the same as the corresponding physical meshes.

Meshes corresponding to $C^{r}$ spaces with stable basis for analysis can be obtained through the macro-element techniques via either the DC or GE method as described 
in Section 3. Figure 14 shows the $C^{r}$ mesh corresponding to $C^{0}$ meshes in Figure 13 , including quadratic $C^{1} \mathrm{PS}$ macro-element space $\mathcal{S}_{2}^{1}\left(T_{p s}\right)$, cubic $C^{1} \mathrm{CT}$ macro-element space $\mathcal{S}_{3}^{1}\left(T_{c t}\right)$, quintic $C^{1}$ polynomial macro-element space $\mathcal{S}_{5}^{1}(T), \mathcal{S}_{5}^{1,2}(T)$ and quintic $C^{2}$ PS macro-element space $\mathcal{S}_{5}^{2}\left(T_{p s}\right)$ and $\mathcal{S}_{5}^{2,3}\left(T_{p s}\right)$. The dimension of each space (number of independent basis functions) is also reported. As can be seen, for $\mathcal{S}_{2}^{1}\left(T_{p s}\right)$ and $\mathcal{S}_{3}^{1}\left(T_{c t}\right)$, although the MDS obtained by DC and GE may be different, the dimension of the spaces are exactly the same, and so are the numerical solutions resulted from the two methods. For the quintic $C^{1}$ and $C^{2}$ spaces, DC yields superspline spaces which have smaller dimension than the ones obtained by GE. For example, the space $\mathcal{S}_{5}^{1,2}(T)$ obtained by DC has dimension 96 (Figure $14(\mathrm{e})$ while the space $\mathcal{S}_{5}^{1}(T)$ obtained by GE has dimension 120 (Figure 14(f)), and the space $\mathcal{S}_{5}^{2,3}\left(T_{p s}\right)$ obtained by DC has dimension 120 (Figure $14(\mathrm{~g})$ while the space $\mathcal{S}_{5}^{2}\left(T_{p s}\right)$ obtained by GE has dimension 252 (Figure 14(h)).

To study the convergence, uniform refinements are performed on the initial $C^{0}$ meshes (2nd row in Figure 13) before the same macro-element techniques are used to obtain stable $C^{r}$ basis. The refinement sequences for these spaces are $\left\{T_{p s}, T_{u, p s}, T_{u, \cdots, u, p s}, \cdots\right\}$ in $\mathcal{S}_{2}^{1}\left(T_{p s}\right),\left\{T_{c t}, T_{u, c t}, T_{u, \cdots, u, c t}, \cdots\right\}$ in $\mathcal{S}_{3}^{1}\left(T_{c t}\right),\left\{T, T_{u}, T_{u, \cdots, u}, \cdots\right\}$ in $\mathcal{S}_{5}^{1,2}(T),\left\{T, T_{u}, T_{u, \cdots, u}, \cdots\right\}$ in $\mathcal{S}_{5}^{1}(T),\left\{T_{p s}, T_{u, p s}, T_{u, \cdots, u, p s}, \cdots\right\}$ in $\mathcal{S}_{5}^{2,3}\left(T_{p s}\right)$ and $\left\{T_{p s}, T_{u, p s}, T_{u, \cdots, u, p s}, \cdots\right\}$ in $\mathcal{S}_{5}^{2}\left(T_{p s}\right)$. Note, in this example, no pre-refinement smooth map was explicitly constructed. The reason for this is that, for domains bounded by straight line segments, the parametric mesh are identical to the physical mesh and the geometric map is in fact $C^{\infty}$ smooth.

In our convergence study, we compute the analysis error by

$$
e_{u}=\left[\int_{\Omega}\left(u_{\text {num }}-u_{\text {exact }}\right) \cdot\left(u_{\text {num }}-u_{\text {exact }}\right) d \Omega\right]^{1 / 2},
$$

where $u_{\text {num }}$ and $u_{\text {exact }}$ are the numerical and exact solutions respectively. The longest edge $h_{\max }$ of the triangles in the physical mesh is considered as the mesh parameter. As shown in Figure 15, optimal convergence rates are achieved in the tested $C^{0}, C^{1}$ and $C^{2}$ spaces, including in the superspline spaces $\mathcal{S}_{5}^{1,2}(T)$ in Figure $15(\mathrm{~b})$ and $\mathcal{S}_{5}^{2,3}\left(T_{p s}\right)$ in Figure 15(c), Particularly, comparing with the regular $C^{r}$ spline space, the same optimal convergence rate is obtained in the superspline space, but with far fewer degrees of freedom. For example, for space $S_{5}^{2}\left(T_{p s}\right)$, the DOF obtained via the GE method are respectively 252, 843, 3063, and 11679, corresponding to the refinement sequences in Fig. 15(c). On the other hand, for the superspline $S_{5}^{2,3}\left(T_{p s}\right)$, the number of DOF obtained via the DC method are respectively 120, 360, 1200, 4320 and 16320, corresponding to the refinement sequences in Fig. $15(\mathrm{c})$. Note that for spaces $\mathcal{S}_{2}^{1}\left(T_{p s}\right)$ and $\mathcal{S}_{3}^{1}\left(T_{c t}\right), \mathrm{DC}$ and GE yields exactly the same analysis results and their convergence curves overlap with each other in this figure.

The refinement steps, the methods for obtaining $C^{r}$ stable basis for analysis, and the convergence rates for each type of elements are summarized in Table 1. It can be seen that optimal convergence rates have been achieved with all types of $C^{r}$ rTBS elements. 


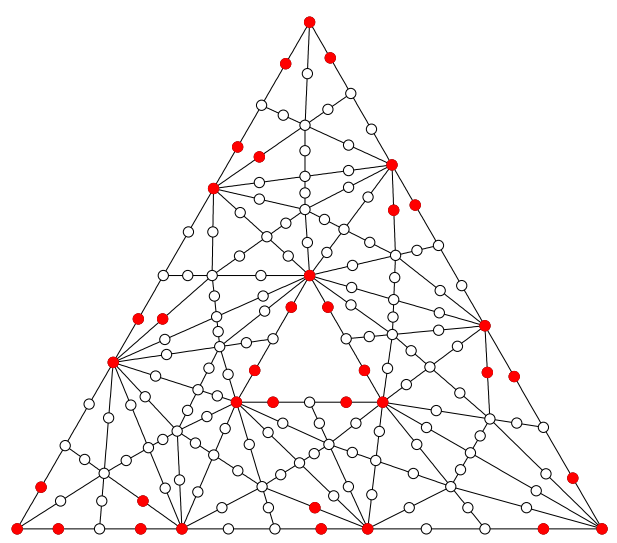

(a) $\mathcal{S}_{2}^{1}\left(T_{p s}\right)$ by DC, $\operatorname{dim}=36$.

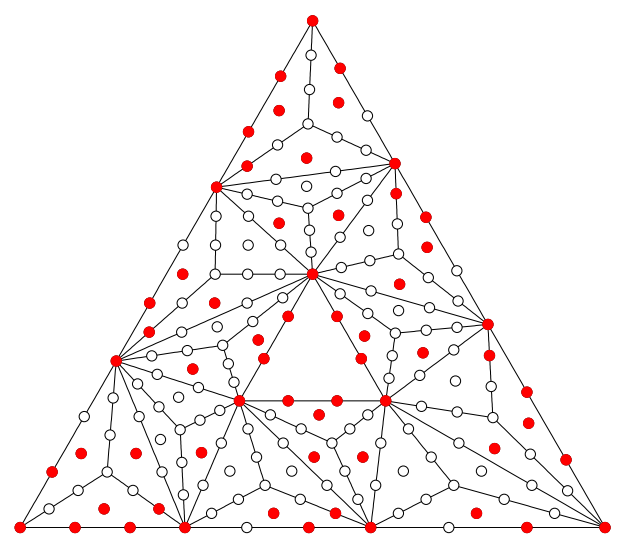

(c) $\mathcal{S}_{3}^{1}\left(T_{c t}\right)$ by DC, $\operatorname{dim}=60$.

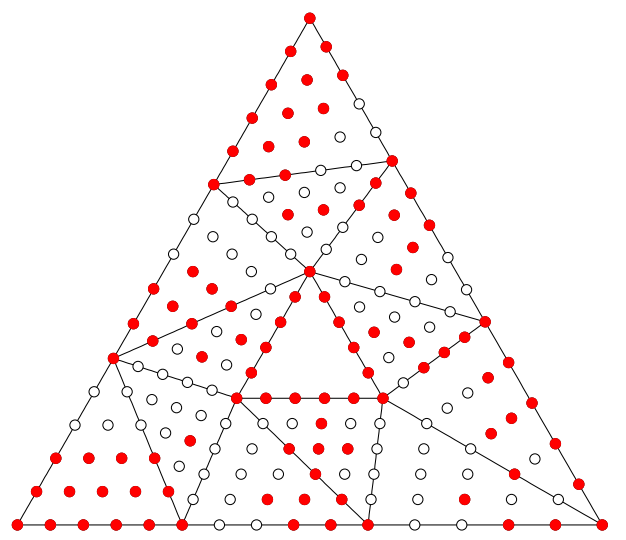

(e) $\mathcal{S}_{5}^{1,2}(T)$ by DC, $\operatorname{dim}=96$.

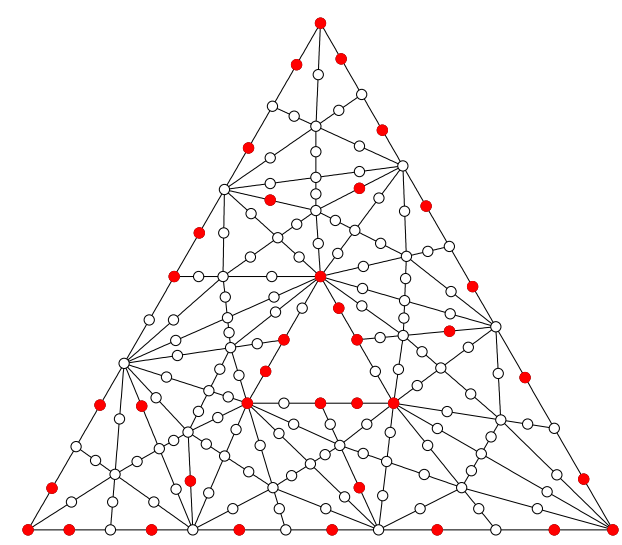

(b) $\mathcal{S}_{2}^{1}\left(T_{p s}\right)$ by GE, $\operatorname{dim}=36$.

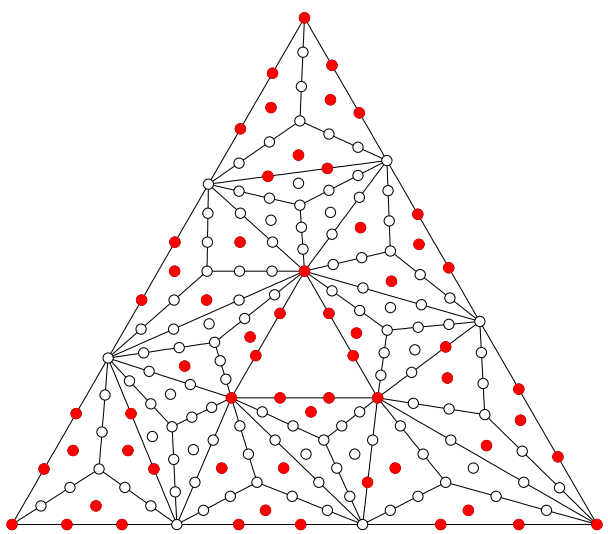

(d) $\mathcal{S}_{3}^{1}\left(T_{c t}\right)$ by GE, dim $=60$.

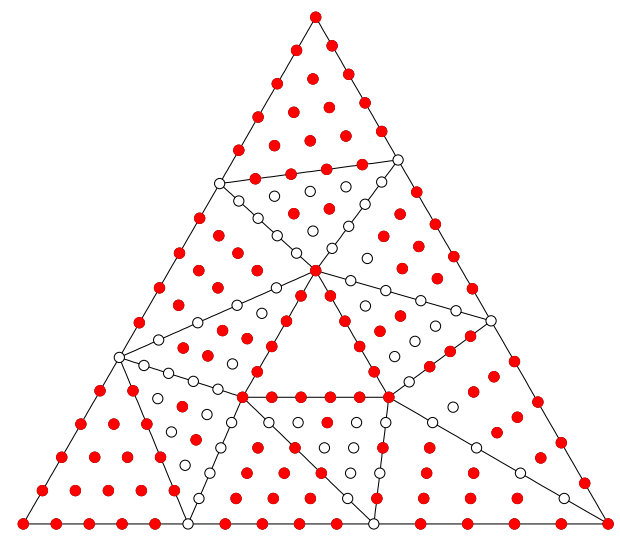

(f) $\mathcal{S}_{5}^{1}(T)$ by $\mathrm{GE}, \operatorname{dim}=120$. 
Table 1: Smooth-refinement-smooth steps and convergence rates of different $C^{r}$ spaces for the problem in Fig. 13

\begin{tabular}{|l|c|c|c|c|c|c|c|}
\hline \multirow{2}{*}{ Step } & \multicolumn{2}{|c|}{ Pre-refinement map } & Refinement & \multicolumn{2}{|c|}{ Stable basis } & Conv. \\
\cline { 2 - 3 } Space & Smoothness & Split & Method & & Split & Method & rate \\
\hline $\mathcal{S}_{2}^{1}\left(T_{p s}\right)$ & $C^{\infty}$ & & & uniform & PS & DC or GE & 3.0 \\
$\mathcal{S}_{3}^{1}\left(T_{c t}\right)$ & $C^{\infty}$ & & & uniform & CT & DC or GE & 3.8 \\
$\mathcal{S}_{5}^{1}(T)$ & $C^{\infty}$ & & & uniform & & GE & 6.0 \\
$\mathcal{S}_{5}^{1,2}(T)$ & $C^{\infty}$ & & & uniform & & DC & 6.0 \\
$\mathcal{S}_{5}^{2}\left(T_{p s}\right)$ & $C^{\infty}$ & & & uniform & PS & GE & 5.8 \\
$\mathcal{S}_{5}^{2,3}\left(T_{p s}\right)$ & $C^{\infty}$ & & & uniform & PS & DC & 5.9 \\
\hline
\end{tabular}

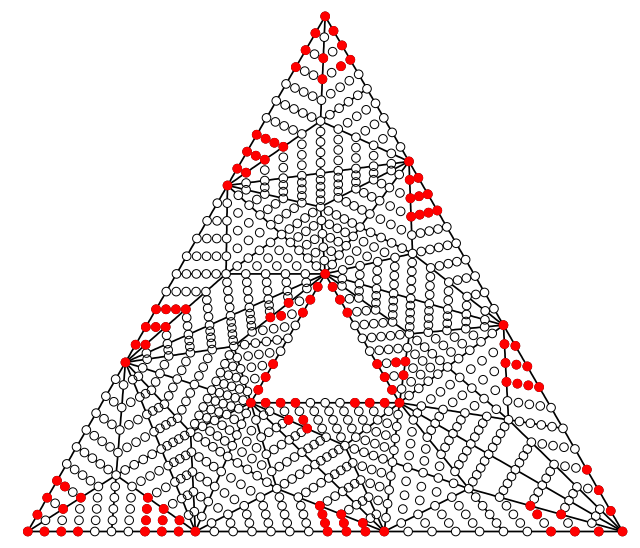

(g) $\mathcal{S}_{5}^{2,3}\left(T_{p s}\right)$ by DC, dim $=120$.

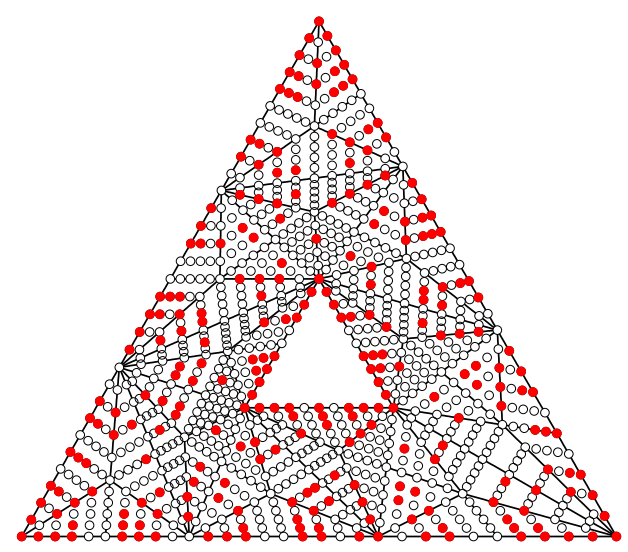

(h) $\mathcal{S}_{5}^{2}\left(T_{p s}\right)$ by GE, $\operatorname{dim}=252$.

Figure 14: Physical meshes (parametric meshes are the same) and dimension for different $C^{r}$ spaces of the problem in Figure 13. Red and white solids represent free and dependent nodes respectively. 


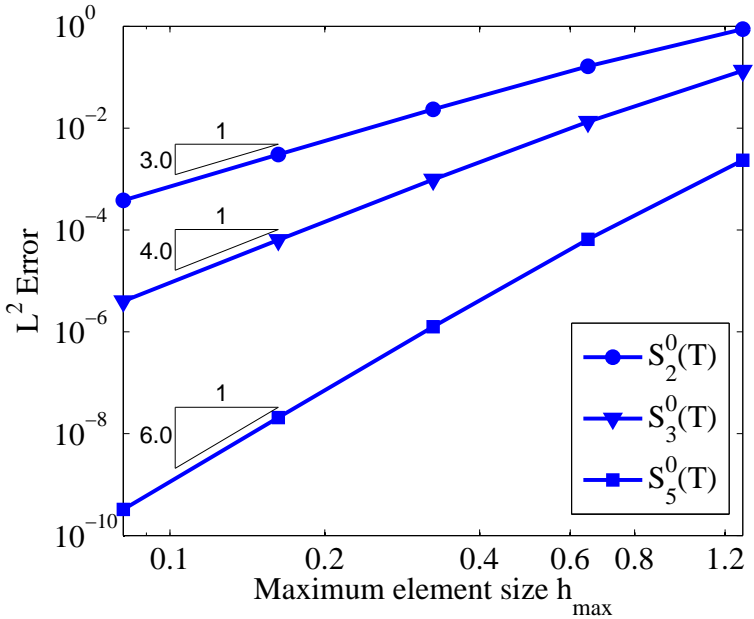

(a) Convergence rates in $C^{0}$ space.

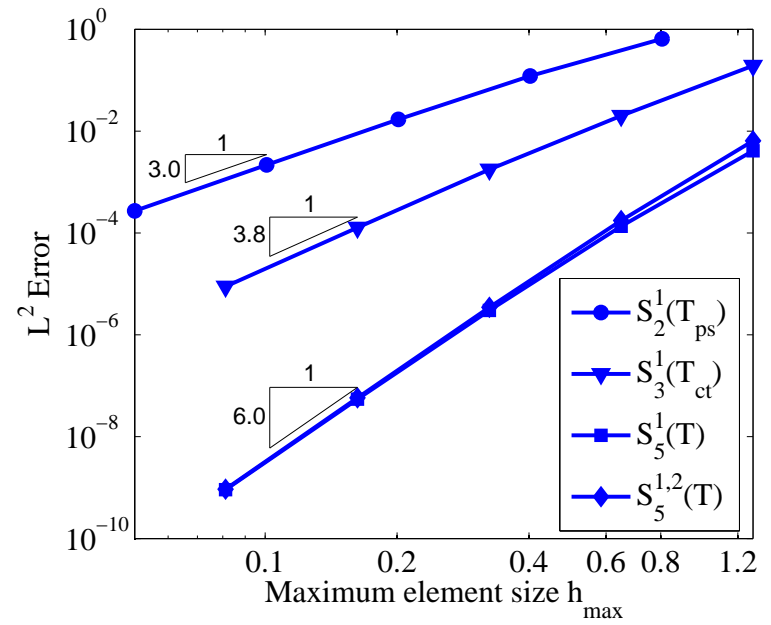

(b) Convergence rates in $C^{1}$ space.

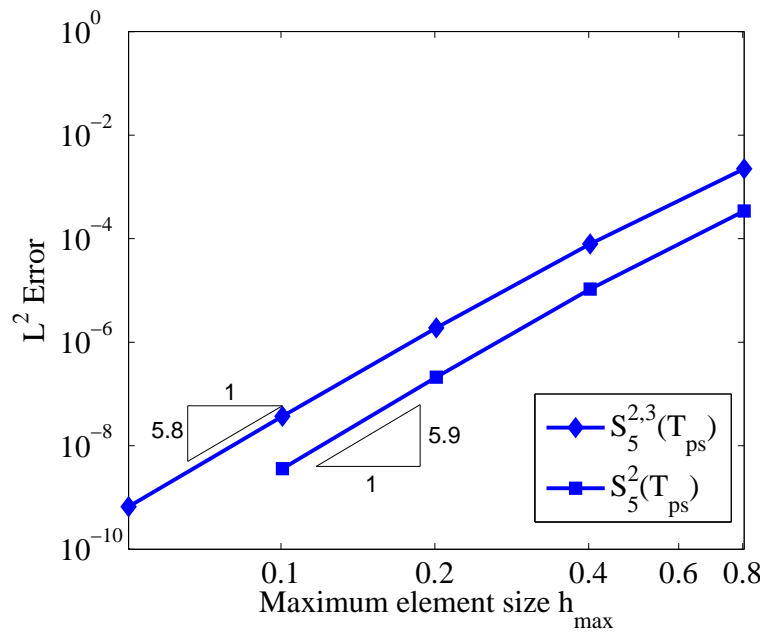

(c) Convergence rates in $C^{2}$ space.

Figure 15: Error measured in the $L^{2}$-norm vs. mesh parameter. Optimal convergence rates are obtained in all shown spaces in Fig. 14 . 


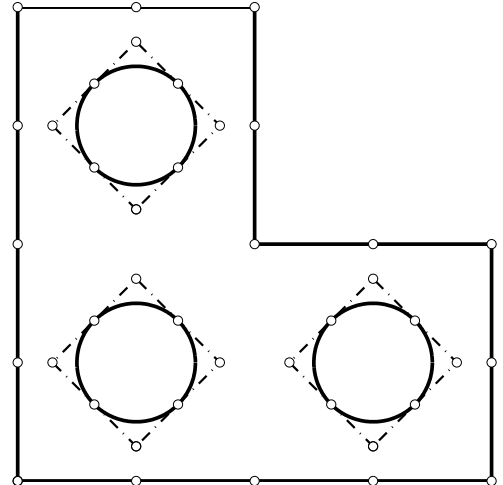

(a) Problem domain NURBS boundary.

\subsection{Domain with curved boundaries: Poisson problem}

In this example we solve a Poisson problem on a L-shaped domain with three holes, as shown in Figure 16(a). The boundaries of the domain are represented in NURBS with weights are so chosen that exact circular holes are represented. The governing equation is (23) with the open set domain $\Omega$ being defined as

$$
\begin{aligned}
\Omega & :=\{(x, y) \mid[(0 \leq x \leq 16) \&(0 \leq y \leq 16)] \backslash[((8<x<16) \&(8<y<16)) \cup \\
& \left.\left.\left((x-4)^{2}+(y-4)^{2}<4\right) \cup\left((x-12)^{2}+(y-4)^{2}<4\right) \cup\left((x-4)^{2}+(y-12)^{2}<4\right)\right]\right\} .
\end{aligned}
$$

The body force is

$$
f(x, y)=2 \sin (x) \sin (y),
$$

and the exact solution is given by

$$
u(x, y)=\sin (x) \sin (y) .
$$

Figure 16: Problem domain and initial parametrization for the Poisson problem. White nodes represents the control points.

To create the initial parametric mesh, we first extract quadratic Bézier curves from the NURBS boundary curves of the physical domain. Particularly, each circular boundary is subtracted as four rational Bézier segments. The end points of these Bézier curves are connected to form the initial parametric domain, which is then triangulated to obtain the initial parametric mesh, as shown in Figure 16(b). We then replace the boundary control points of the parametric mesh with corresponding points from the physical boundary to obtain the initial physical mesh, as shown in Figure 16(c). The $C^{r}$ stable basis for analysis can be obtained as usual by degree elevation followed by either the DC or GE method based on the macro-element techniques. Figure 17 shows the meshes corresponding to quadratic $C^{1}$ PS macro-element space $\mathcal{S}_{2}^{1}\left(T_{p s}\right)$, cubic $C^{1}$ CT macro-element space $\mathcal{S}_{3}^{1}\left(T_{c t}\right)$, quintic $C^{1}$ polynomial macro-element space $\mathcal{S}_{5}^{1}(T)$ and $\mathcal{S}_{5}^{1,2}(T)$.

The refinement sequences used to evaluate the convergence are $\left\{T_{p s}, T_{p s, u, p s}, T_{p s, u, \cdots, u, p s}, \cdots\right\}$ in $\mathcal{S}_{2}^{1}\left(T_{p s}\right),\left\{T_{c t}, T_{c t, u, c t}, T_{c t, u, \cdots, u, c t}, \cdots\right\}$ in $\mathcal{S}_{3}^{1}\left(T_{c t}\right),\left\{T, T_{u}, T_{u, \cdots, u}, \cdots\right\}$ in $\mathcal{S}_{5}^{1}(T)$, and $\left\{T_{p s}, T_{p s, u}, T_{p s, u, \cdots, u}, \cdots\right\}$ in $\mathcal{S}_{5}^{1,2}(T)$. The methods for constructing pre-refinement smooth maps, refinement, and methods of basis construction in each $C^{r}$ space are summarized in Table 2. For example, for the superspline space $\mathcal{S}_{5}^{1,2}(T)$, the initial physical mesh is $C^{2}$ smooth obtained by GE with PS macro-element. The four rows in Figure 17 correspond 
to the four initial pairs of parametric and physical meshes in the four convergence studies respectively. The convergence rates obtained in $C^{0}$ and $C^{1}$ spaces are all optimal as shown in Figure 18(a) and 18(b), where the convergence rates for quadratic, cubic and quintic elements are 3,4 ,and 6 respectively in all $C^{0}, C^{1}$ and $\mathcal{S}^{1,2}$ spaces.

Table 2: Smooth-refinement-smooth steps and convergence rates of different $C^{r}$ spaces for the problem in Fig. 16 ,

\begin{tabular}{|l|c|c|c|c|c|c|c|}
\multirow{2}{*}{ Step } & \multicolumn{2}{|c|}{ Pre-refinement map } & Refinement & \multicolumn{2}{|c|}{ Stable basis } & Conv. \\
\cline { 2 - 4 } Space & Smoothness & Split & Method & & Split & Method & rate \\
\hline $\mathcal{S}_{2}^{1}\left(T_{p s}\right)$ & $C^{1}$ & PS & DC & uniform & PS & DC & 3.1 \\
$\mathcal{S}_{3}^{1}\left(T_{c t}\right)$ & $C^{1}$ & CT & DC & uniform & CT & DC & 3.9 \\
$\mathcal{S}_{5}^{1}(T)$ & $C^{1}$ & & GE & uniform & & GE & 6.0 \\
$\mathcal{S}_{5}^{1,2}(T)$ & $C^{2}$ & PS & GE & uniform & & DC & 5.7 \\
\hline
\end{tabular}

If the refine-then-smooth strategy is used, that is, no smooth pre-refinement map is constructed, the geometric map changes and convergence rate decreases as the mesh is refined. As shown in Figure 19, although the rate in $\mathcal{S}_{2}^{1}\left(T_{p s}\right)$ is optimal, the rates in $\mathcal{S}_{3}^{1}\left(T_{c t}\right)$ and $\mathcal{S}_{5}^{1}(T)$ are only 3 , and the rate in $\mathcal{S}_{5}^{1,2}(T)$ decreases quickly to about 4.1 . Note that the extreme large errors in $\mathcal{S}_{5}^{1,2}(T)$ for the coarse meshes are due to the poor mesh quality. 


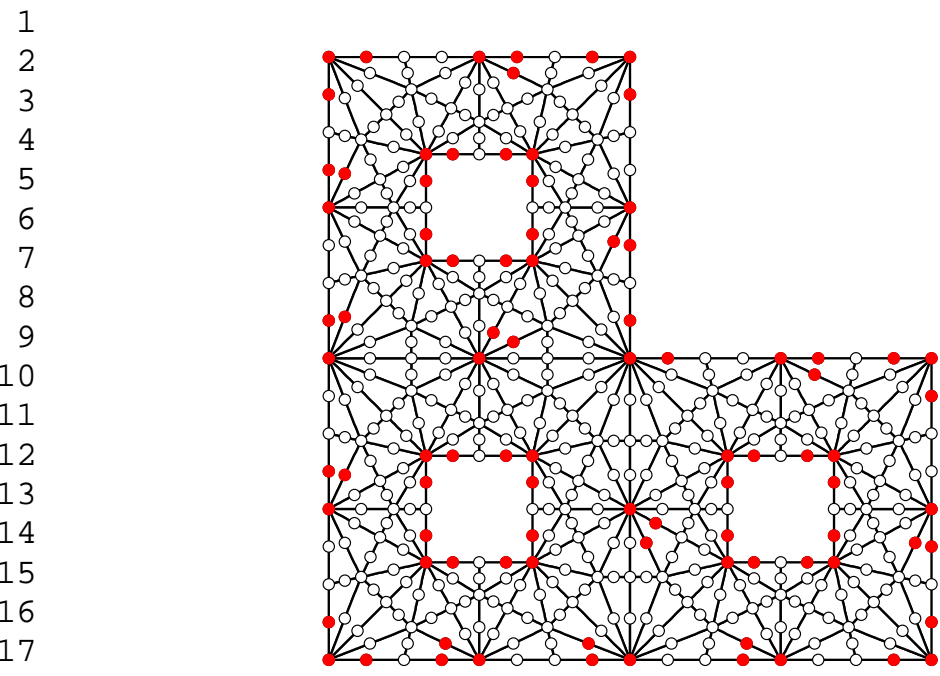

(a) Parametric mesh in $\mathcal{S}_{2}^{1}\left(\widehat{T}_{p s}\right)$ with basis obtained by DC with PS macro-elements.

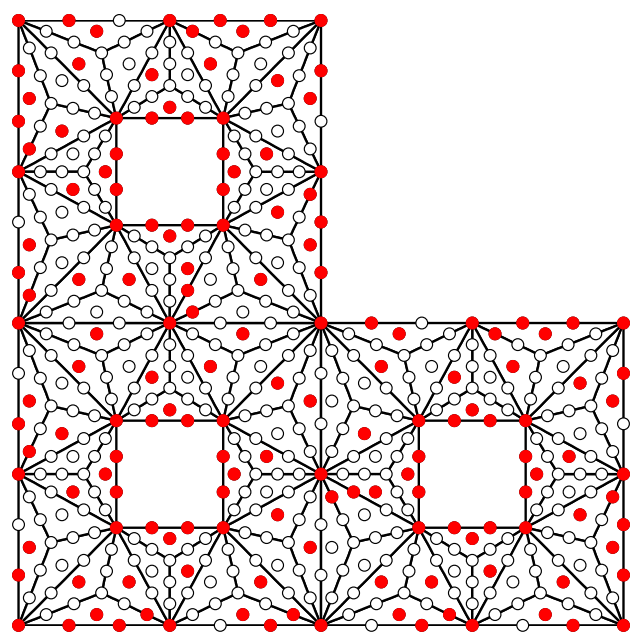

(c) Parametric mesh in $\mathcal{S}_{3}^{1}\left(\widehat{T}_{c t}\right)$ with basis obtained by DC with CT macro-elements.

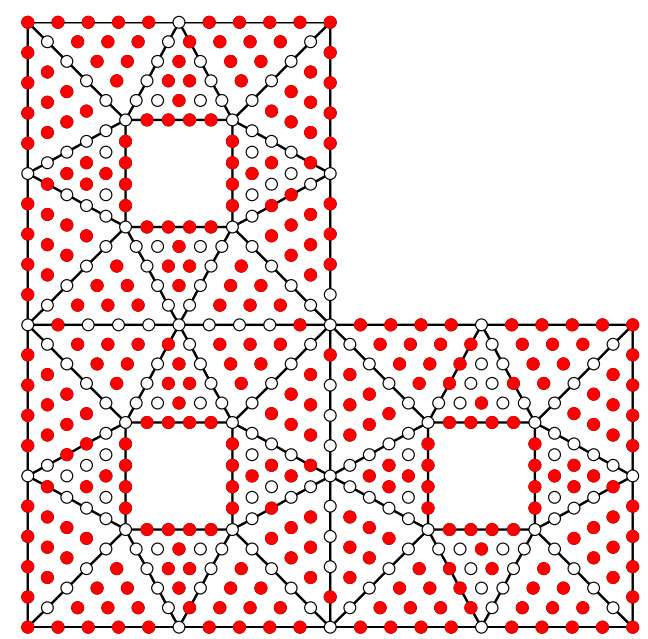

(e) Parametric mesh in $\mathcal{S}_{5}^{1}(\widehat{T})$ with basis obtained by GE.

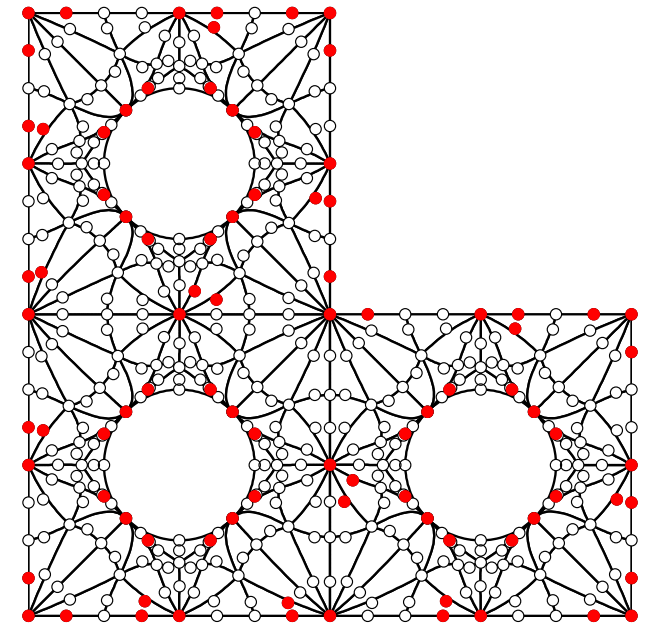

(b) $C^{1}$ physical mesh obtained by $\mathrm{DC}$ with PS macro-elements.

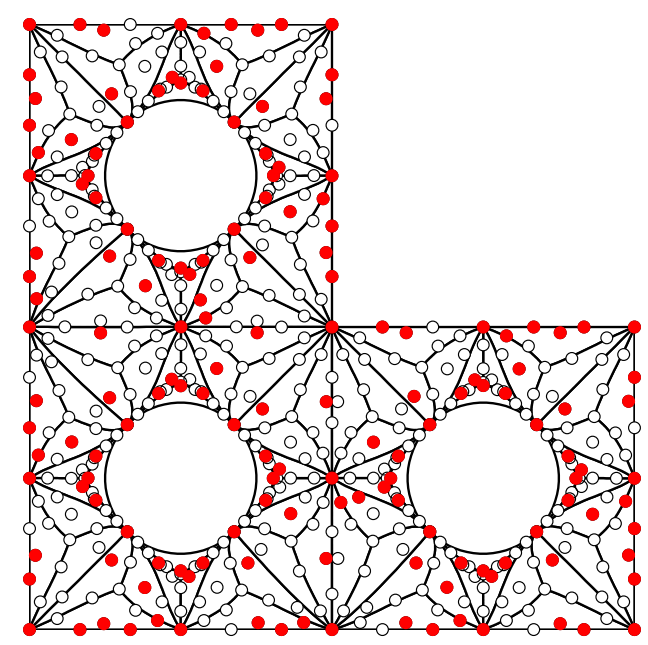

(d) $C^{1}$ physical mesh obtained by $\mathrm{DC}$ with CT macro-elements.

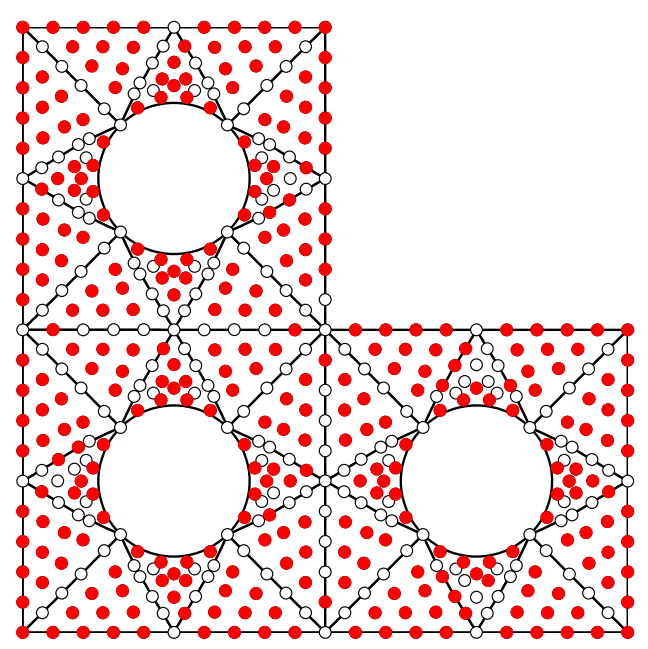

(f) $C^{1}$ physical mesh obtained by GE. 


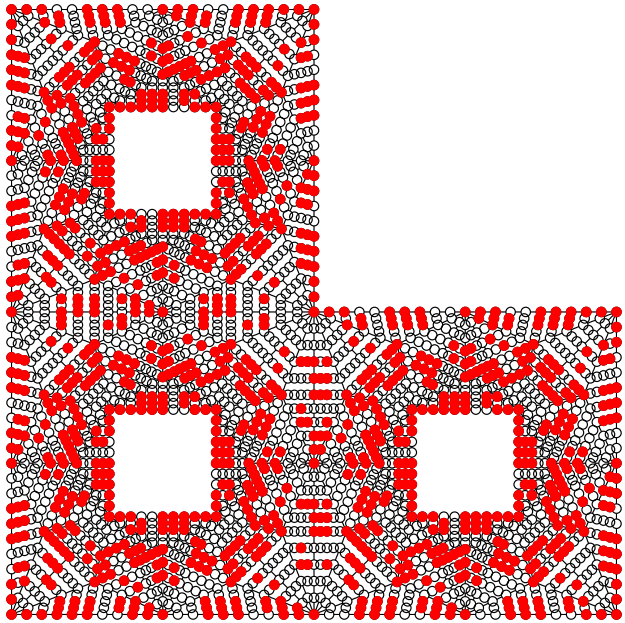

(g) Parametric mesh in $\mathcal{S}_{5}^{1,2}(\widehat{T})$ with basis obtained by DC.

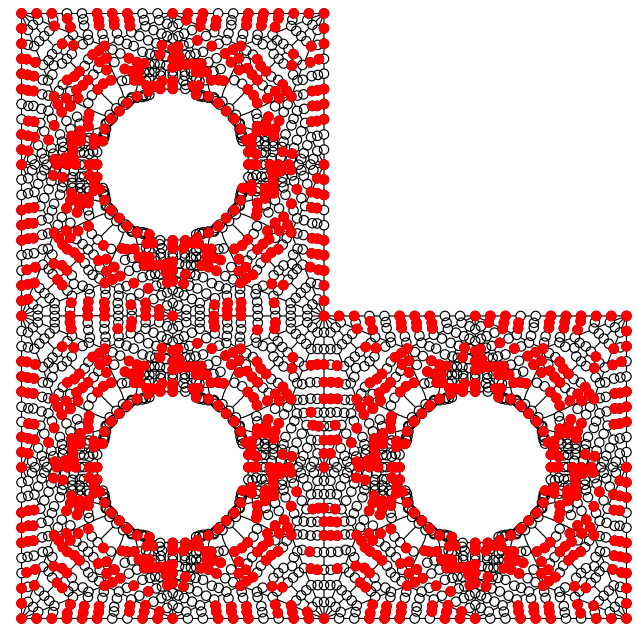

(h) Corresponding $C^{2}$ physical mesh in $\mathcal{S}_{5}^{1,2}(\widehat{T})$.

Figure 17: Parametric and physical meshes for different $C^{r}$ spaces. Each row shows the first parametric and corresponding $C^{1}$ (first three rows) or $C^{2}$ (fourth row) physical mesh in each refinement sequence for the problem in Fig. 16. Red and white solids represent free and dependent nodes respectively.

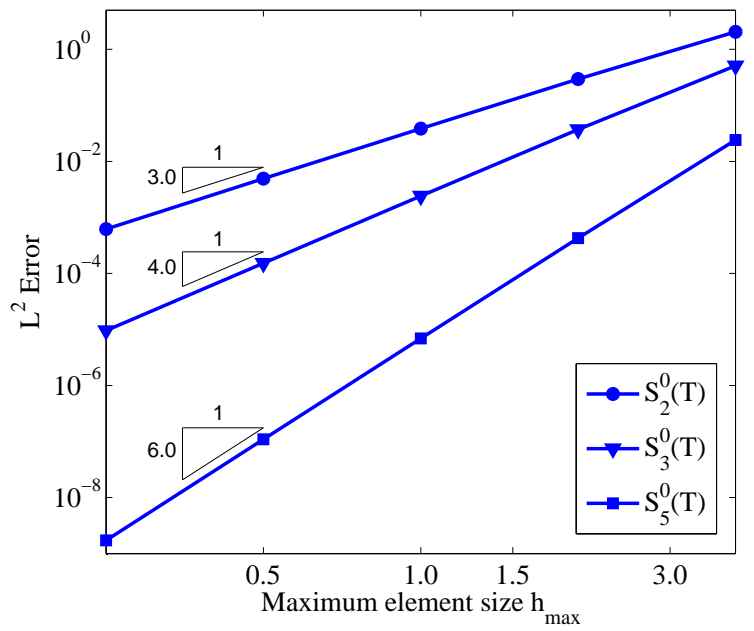

(a) Convergence rates in $C^{0}$ space.

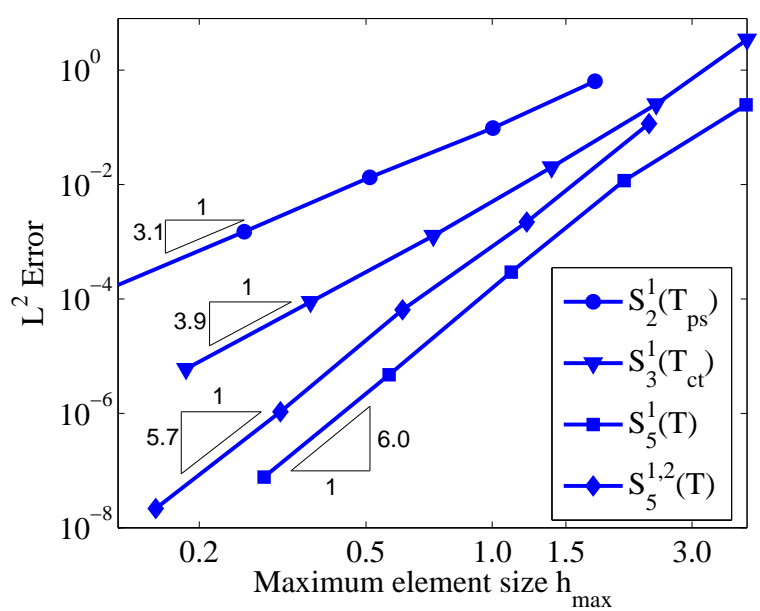

(b) Convergence rates in $C^{1}$ space with nested refinement sequences.

Figure 18: Error measured in the $L^{2}$-norm vs. mesh parameter from refined elements in Fig. 17. 


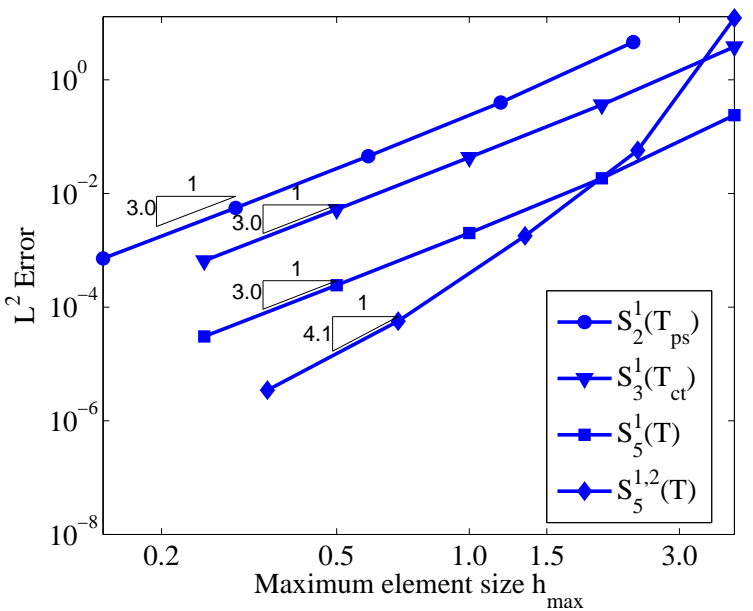

Figure 19: Error measured in the $L^{2}$-norm vs. mesh parameter in $C^{1}$ spaces for refinement sequences with inconsistent geometric map. The convergence rates decrease as the meshes are refined. 


\subsection{Domain with curved boundaries: linear elasticity}

In the third example, we apply our approach to a well-known linear elasticity problem: an infinite plate with a circular hole under constant in-plane tension in the $x$-direction [1. The infinite plate is modeled by a finite quarter plate as shown in Figure 20 with the governing equation (22). The exact solution [53], evaluated at the boundary of the finite quarter plate, is applied as a Neumann boundary condition.

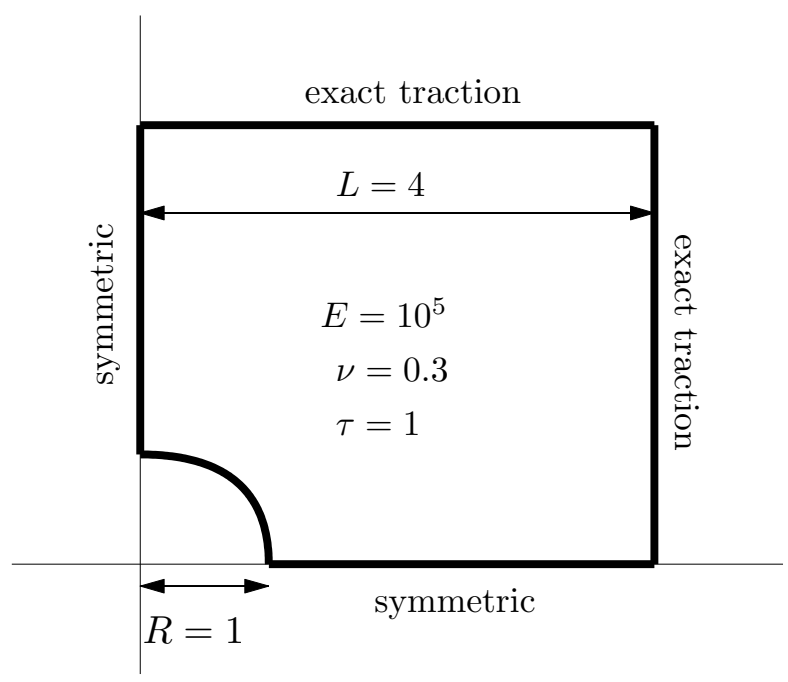

Figure 20: Problem definition: elastic plate with a circular hole. $L$ is the length of the edge, $R$ is the radius of the circle and $\tau$ is the thickness of the plate. $E$ and $\nu$ represent the Young's modulus and Poisson ratio respectively.

The initial parametrization of the physical domain is shown in Figure 21. The NURBS boundary curves of the given domain are first extracted as rational quadratic Bézier curves. After connecting the end points of the Bézier curves we obtain an initial parametric domain and triangulate it, as shown in Figure 21(b). Then we replace the boundary control points with corresponding points on the physical boundary to obtain the initial physical mesh as shown in Figure 21(a). To improve the mesh quality and analysis results, we use the same smoothed parametric mesh (Figure 21(c)] as in [38] by minimizing the difference of the internal corner angles between the parametric and physical domains.

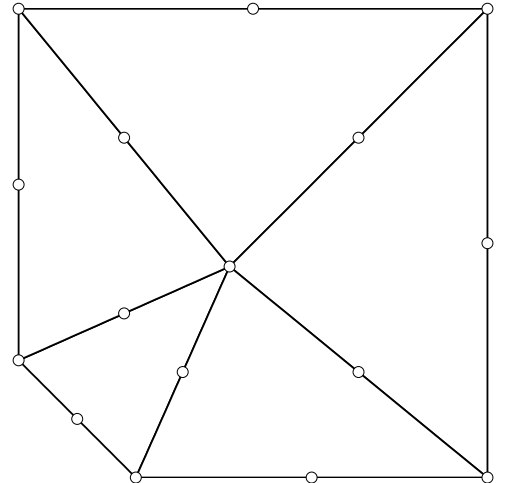

(a) Initial parametric mesh.

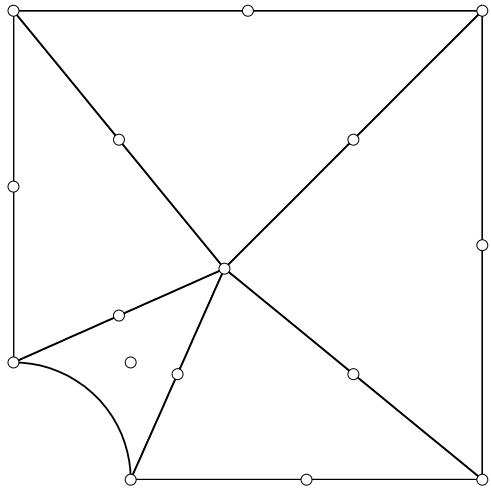

(b) Inital physical mesh.

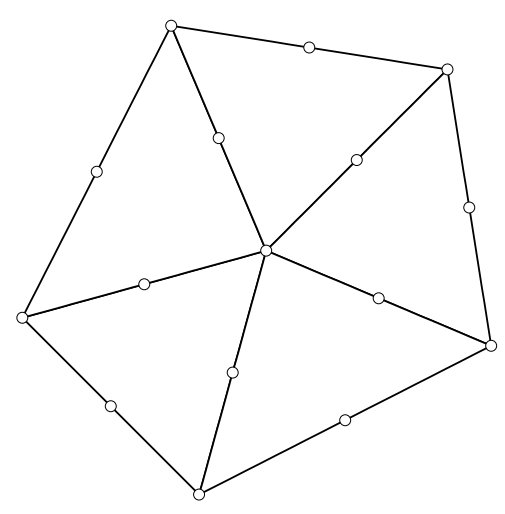

(c) Smoothed parametric mesh.

Figure 21: Initial parametrization of the physical domain in Figure 20.

Through degree elevation on the parametric and physical meshes in Figure 21, we obtain cubic and quintic $C^{0}$ meshes in $\mathcal{S}_{3}^{0}(T)$ and $\mathcal{S}_{5}^{0}(T)$, as shown in Figure 22, Meshes 
in $C^{r}$ spaces for analysis are also obtained by either DC or GE method, as shown in Figure 22,

The refinement sequences used to evaluate the convergence are $\left\{T, T_{u}, T_{u, \cdots, u}, \cdots\right\}$ in all $C^{0}$ spaces, $\left\{T_{p s}, T_{p s, u, p s}, T_{p s, u, \cdots, u, p s}, \cdots\right\}$ in $\mathcal{S}_{2}^{1}\left(T_{p s}\right),\left\{T_{c t}, T_{c t, u, c t}, T_{c t, u, \cdots, u, c t}, \cdots\right\}$ in $\mathcal{S}_{3}^{1}\left(T_{c t}\right),\left\{T, T_{u}, T_{u, \cdots, u}, \cdots\right\}$ in $\mathcal{S}_{5}^{1,2}(T),\left\{T, T_{u}, T_{u, \cdots, u}, \cdots\right\}$ in $\mathcal{S}_{5}^{1}(T),\left\{T_{p s}, T_{u, p s}, T_{u, \cdots, u, p s}, \cdots\right\}$ in $\mathcal{S}_{5}^{2,3}\left(T_{p s}\right)$ and $\mathcal{S}_{5}^{2}\left(T_{p s}\right)$. The steps of convergence analysis and methods of basis construction in each $C^{r}$ space is summarized in Table 3 . The four rows in Figure 22 correspond to the four initial pairs of parametric and physical meshes in the four convergence studies respectively. Note, , the pre-refinement smooth geometric map, $C^{2}$ and $C^{3}$ used in space $\mathcal{S}_{5}^{2}\left(T_{p s}\right)$ and $\mathcal{S}_{5}^{2,3}\left(T_{p s}\right)$ are both obtained by GE after imposing continuity constraints on the quintic $C^{0}$ mesh.

Table 3: Smooth-refinement-smooth steps and convergence rates of different $C^{r}$ spaces for the problem in Fig. 20

\begin{tabular}{|l|c|c|c|c|c|c|c|}
\hline \multicolumn{1}{|c|}{ Step } & \multicolumn{2}{|c|}{ Pre-refinement map } & Refinement & \multicolumn{2}{|c|}{ Stable basis } & Conv. \\
\cline { 2 - 4 } Space & Smoothness & Split & Method & & Split & Method & rate \\
\hline $\mathcal{S}_{2}^{1}\left(T_{p s}\right)$ & $C^{1}$ & PS & DC & uniform & PS & DC & 2.0 \\
$\mathcal{S}_{3}^{1}\left(T_{c t}\right)$ & $C^{1}$ & CT & DC & uniform & CT & DC & 2.8 \\
$\mathcal{S}_{5}^{1}(T)$ & $C^{1}$ & & GE & uniform & & GE & 5.0 \\
$\mathcal{S}_{5}^{1,2}(T)$ & $C^{2}$ & & GE & uniform & & DC & 5.0 \\
$\mathcal{S}_{5}^{2}\left(T_{p s}\right)$ & $C^{2}$ & & GE & uniform & PS & GE & 5.0 \\
$\mathcal{S}_{5}^{2,3}\left(T_{p s}\right)$ & $C^{3}$ & & GE & uniform & PS & DC & 4.7 \\
\hline
\end{tabular}

The energy error is evaluated by

$$
e_{\text {stress }}=\left[\frac{1}{2} \int_{\Omega}\left(\varepsilon_{\text {num }}-\varepsilon_{\text {exact }}\right) \cdot \mathbf{D} \cdot\left(\varepsilon_{\text {num }}-\varepsilon_{\text {exact }}\right) d \Omega\right]^{1 / 2},
$$

where $\varepsilon_{\text {num }}$ and $\varepsilon_{\text {exact }}$ are the numerical and exact strain vectors respectively. The mesh parameter is evaluated as the longest edge $h_{\max }$ of the triangles in the physical mesh. Again optimal convergence rates are achieved in $C^{0}, C^{1}$ and $C^{2}$ spaces, as shown in Figure 23 where quadratic, cubic and quintic rates are obtained for the energy norm error using quadratic, cubic and quintic elements respectively. In the superspline spaces $\mathcal{S}_{5}^{1,2}(T)$ and $\mathcal{S}_{5}^{2,3}\left(T_{p s}\right)$, optimal rates are also observed as shown in Figure 23(b) and 23(c). This demonstrates the efficiency of supersplines for analysis on a per-node basis since far fewer degrees of freedom are used in superspline space than the regular spline space of the same degree. Figure 24 plots the convergence curve on a node basis. It can be seen that at the same number of nodes in three quintic elements, $S_{5}^{0}, S_{5}^{1}, S_{5}^{2,3}$, higher continuity leads to smaller errors.

However, if we use the refine-then-smooth strategy, that is, without constructing the smooth pre-refinement map, the local modification of the control points to obtain the $C^{r}$ basis would change the geometric map. Consequently the convergence rates are reduced, as shown in Figure 25. When the refinements are started from $C^{0}$ meshes, the convergence rates in all $C^{1}$ and $C^{2}$ spaces with polynomial degrees ranging from quadratic to quintic are about the same, which range from 1.4 to 1.6 and are far from the optimal values. When the refinements are started from $C^{1}$ and $C^{2}$ meshes in spaces $\mathcal{S}_{5}^{1,2}$ and $\mathcal{S}_{5}^{2,3}$ respectively, the convergence rates become closer to the optimal values, since the geometric map do not change as much as when start refining from $C^{0}$ meshes (see Figure 11). This is another evidence showing the change of geometric map is affecting the convergence rates. 


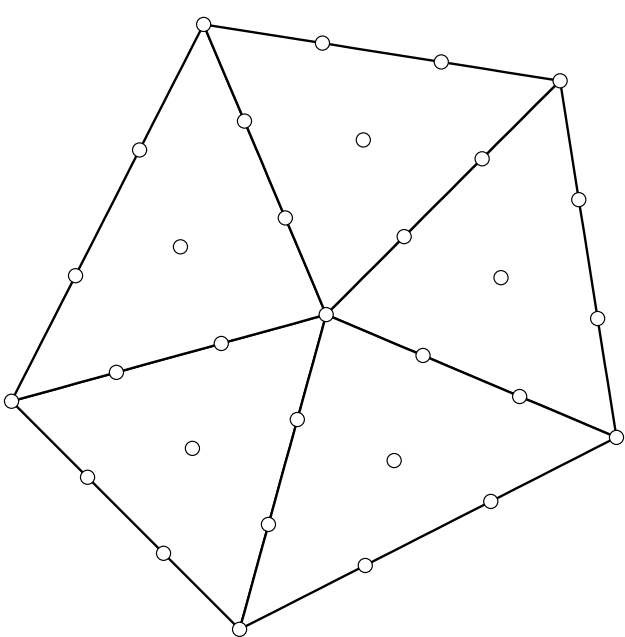

(a) Parametric mesh in $\mathcal{S}_{3}^{0}(\widehat{T})$.

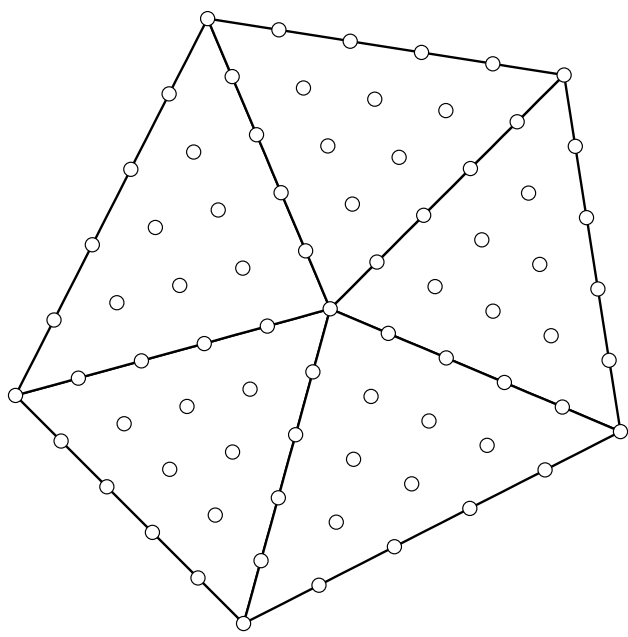

(c) Parametric mesh in $\mathcal{S}_{5}^{0}(\widehat{T})$.

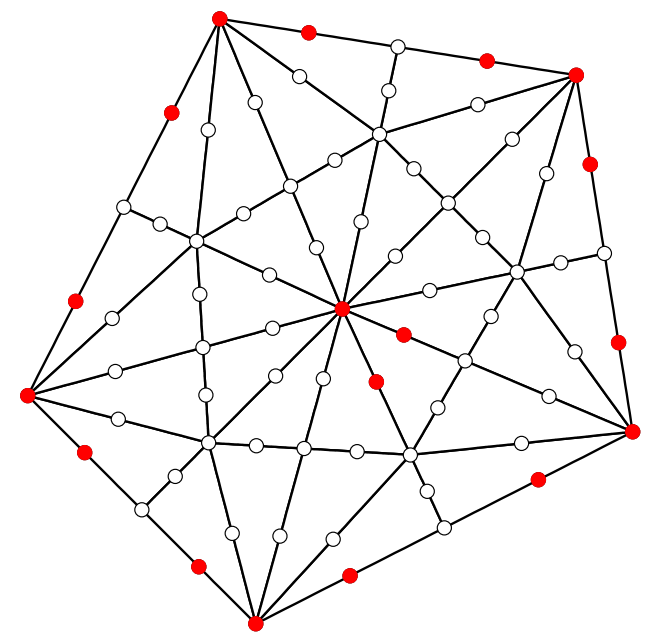

(e) Parametric mesh in $\mathcal{S}_{2}^{1}\left(\widehat{T}_{p s}\right)$ with basis obtained by DC with PS macro-elements.

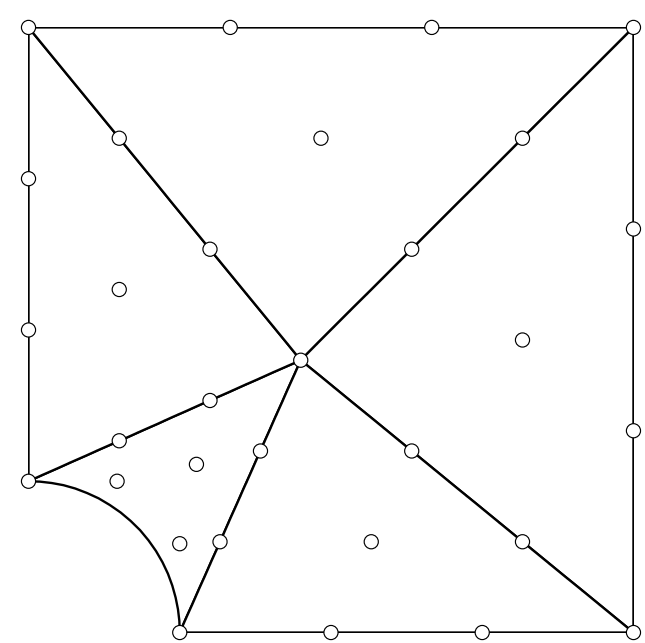

(b) Physical mesh in $\mathcal{S}_{3}^{0}(T)$.

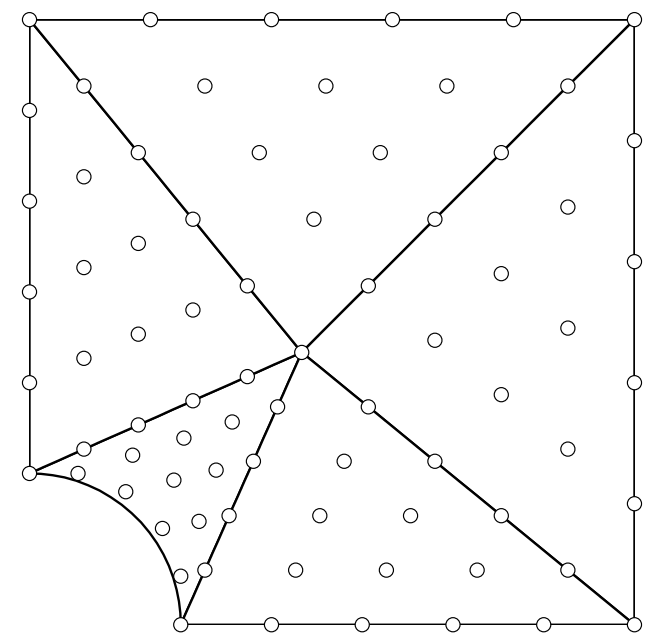

(d) Physical mesh in $\mathcal{S}_{5}^{0}(T)$.

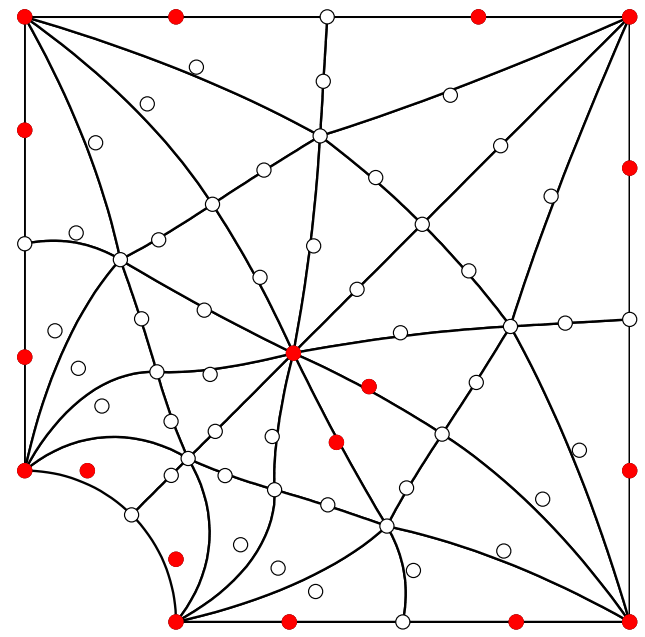

(f) $C^{1}$ physical mesh obtained by $\mathrm{DC}$ with PS macro-elements. 


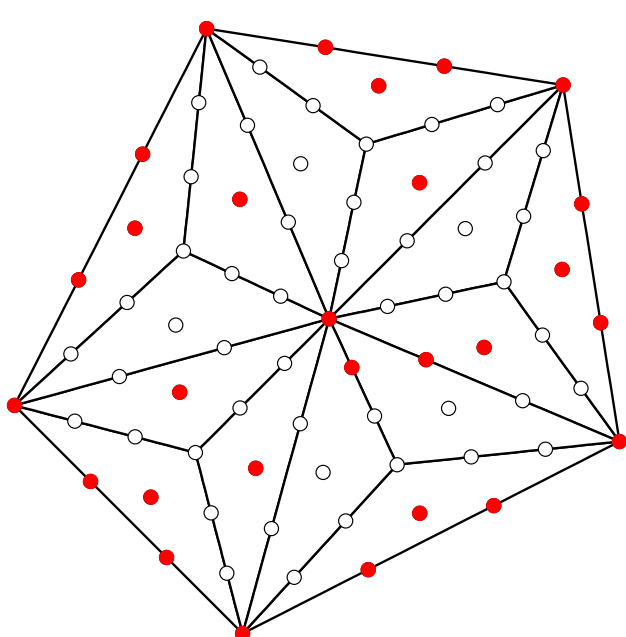

(g) Parametric mesh in $\mathcal{S}_{3}^{1}\left(\widehat{T}_{c t}\right)$ with basis obtained by DC with CT macro-elements.

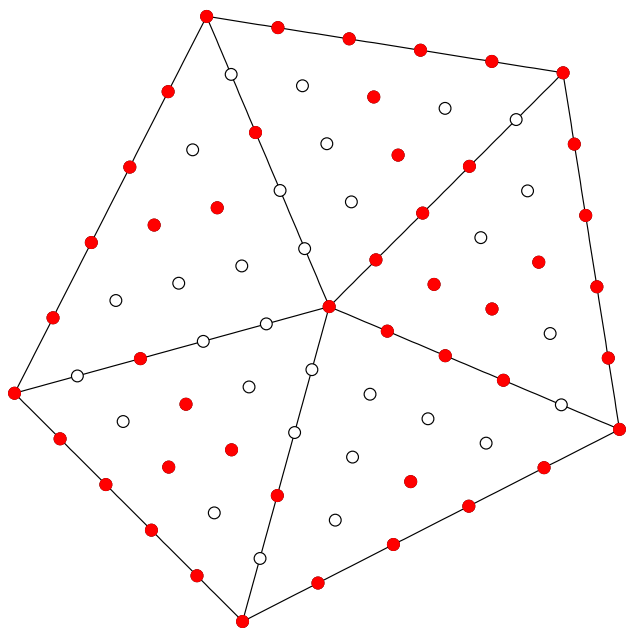

(i) Parametric mesh in $\mathcal{S}_{5}^{1,2}(\widehat{T})$ with basis obtained by DC.

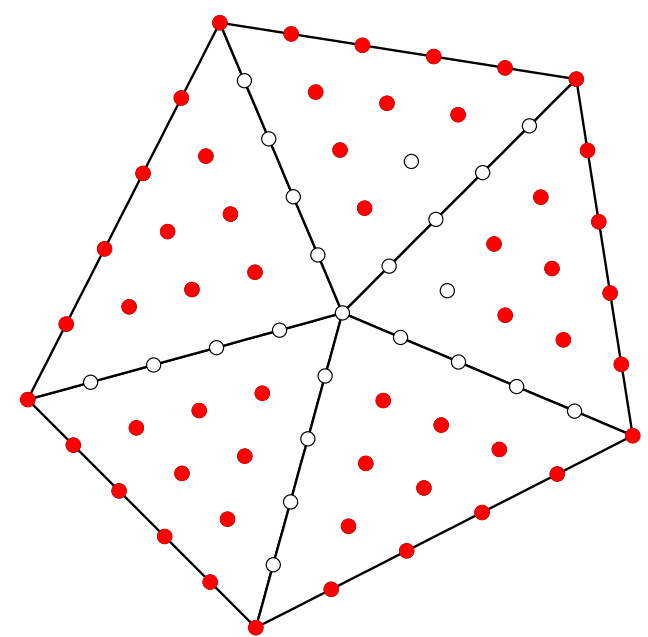

(k) Parametric mesh in $\mathcal{S}_{5}^{1}(\widehat{T})$ with basis obtained by GE.

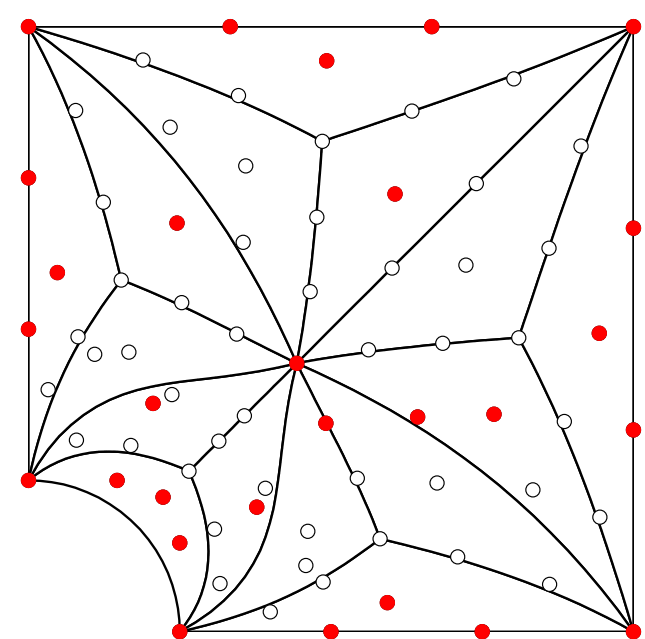

(h) $C^{1}$ physical mesh obtained by DC with CT macro-elements.

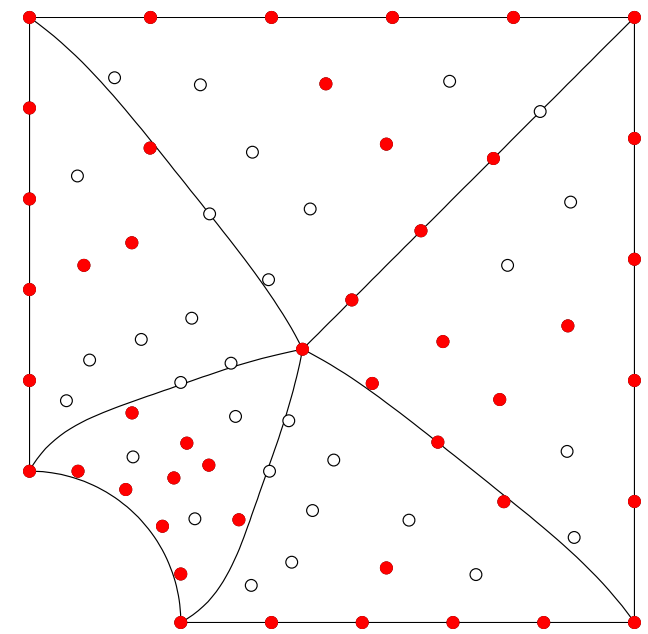

(j) Corresponding $C^{2}$ physical mesh in $\mathcal{S}_{5}^{1,2}(T)$ obtained by GE.

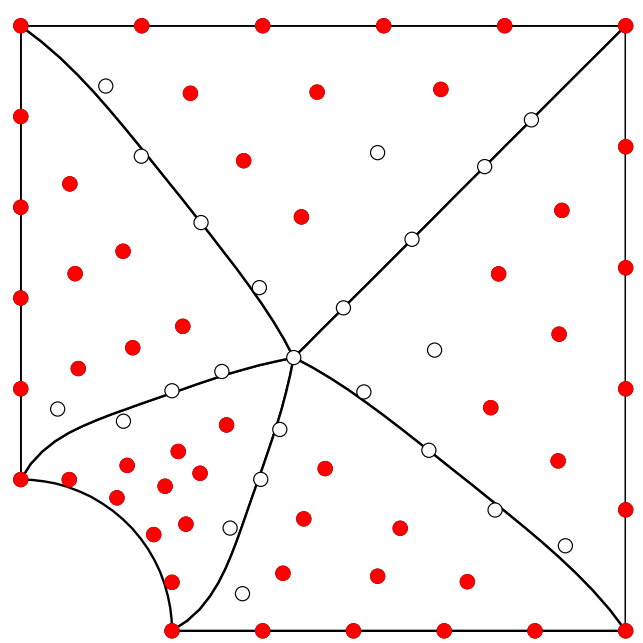

(l) Corresponding $C^{1}$ physical mesh in $\mathcal{S}_{5}^{1}(T)$ obtained by GE. 


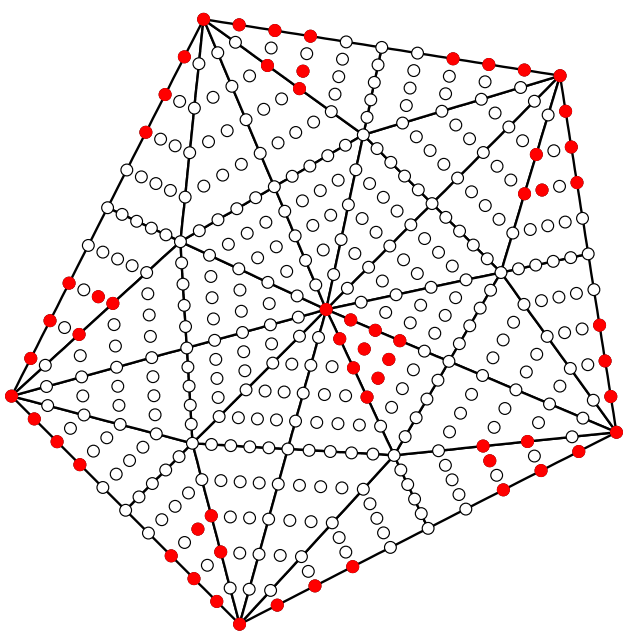

(m) Parametric mesh in $\mathcal{S}_{5}^{2,3}\left(\widehat{T}_{p s}\right)$ with basis obtained by DC with PS macroelements.

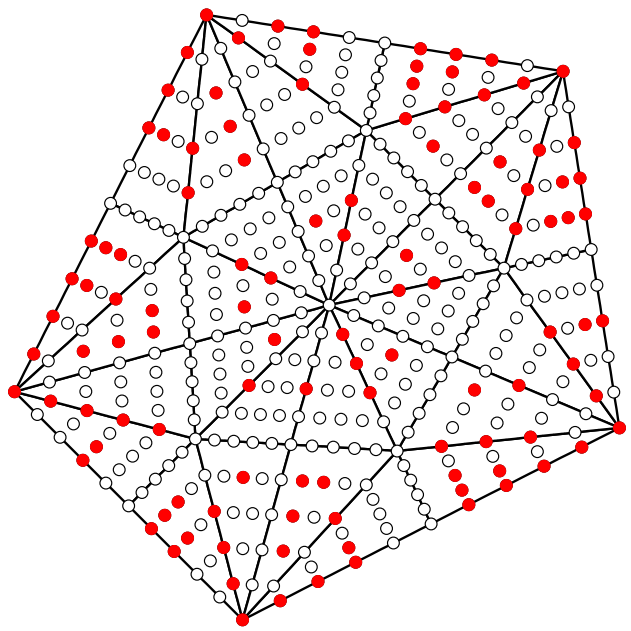

(o) Parametric mesh in $\mathcal{S}_{5}^{2}\left(\widehat{T}_{p s}\right)$ with basis obtained by GE with PS macro-elements.

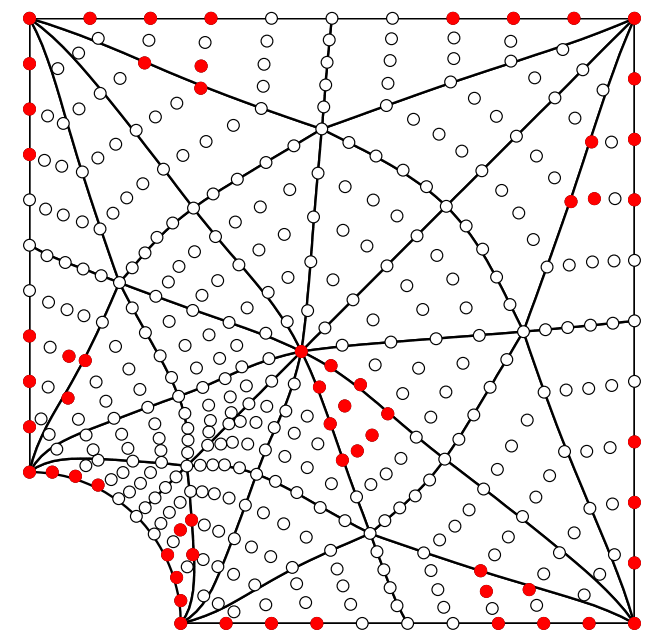

(n) Corresponding $C^{3}$ physical mesh in $\mathcal{S}_{5}^{2,3}\left(T_{p s}\right)$ obtained by GE.

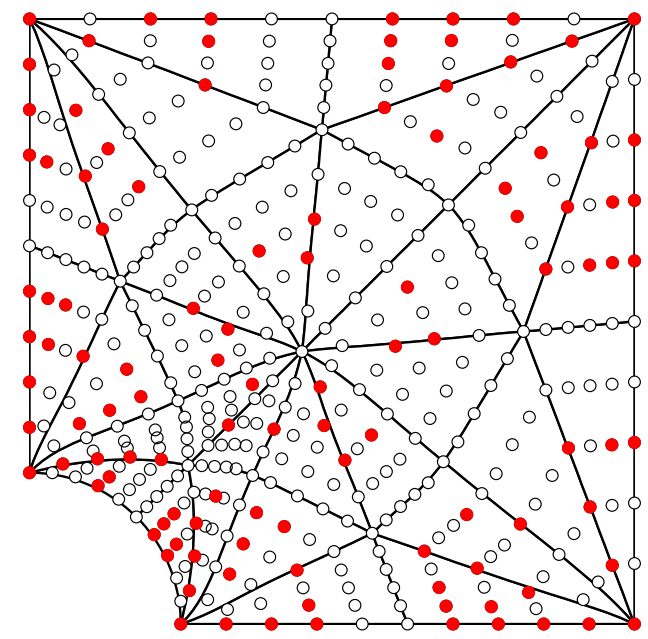

(p) Corresponding $C^{2}$ physical mesh in $\mathcal{S}_{5}^{2}\left(T_{p s}\right)$ obtained by GE.

Figure 22: Parametric and physical meshes in different $C^{r}$ spaces. Each row shows the first parametric and corresponding $C^{r}$ physical mesh in each refinement sequence for the problem in Fig. 20. Red and white solids represent free and dependent nodes respectively. 


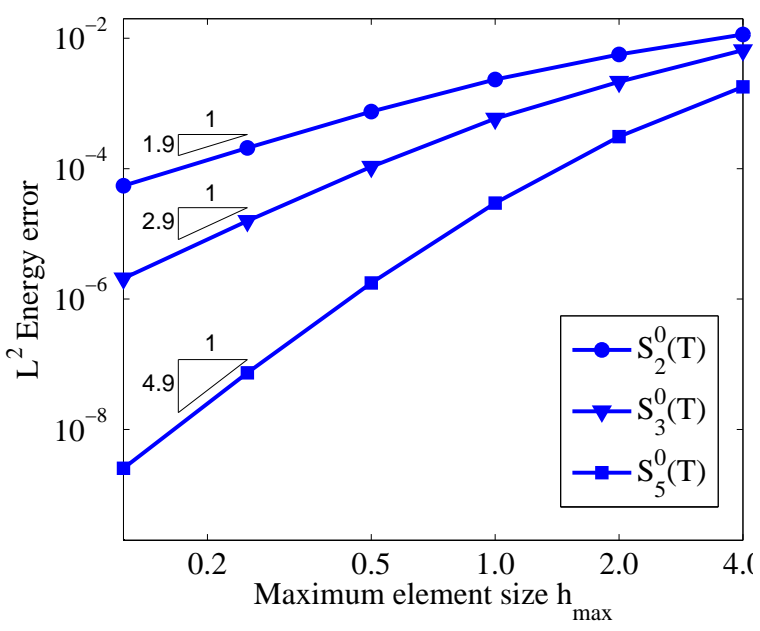

(a) Convergence rates in $C^{0}$ space.

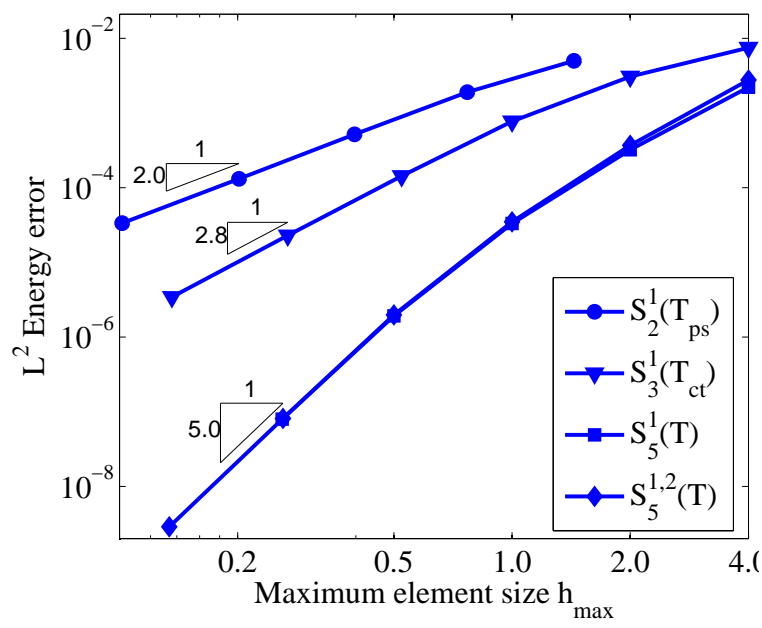

(b) Convergence rates in $C^{1}$ space.

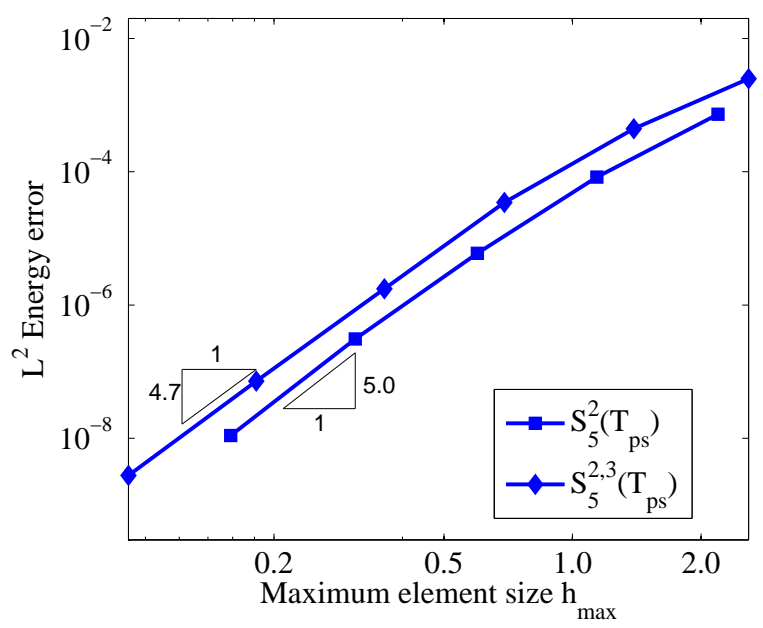

(c) Convergence rates in $C^{2}$ space $\mathcal{S}_{5}^{2}\left(\widehat{T}_{p s}\right)(\mathrm{GE})$ and superspline space $\mathcal{S}_{5}^{2,3}\left(\widehat{T}_{p s}\right)$ (DC).

Figure 23: Error measured in the $L^{2}$-norm of stress vs. mesh parameter from refinements of elements in Fig. 22. Optimal convergence rates are obtained in all cases.

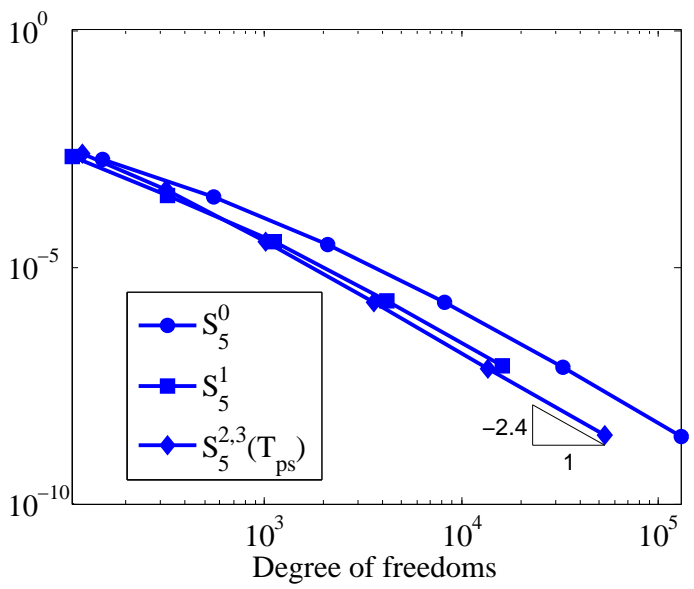

Figure 24: Error measured in the $L^{2}$-norm of stress vs. the number of nodes from refinements of elements in Fig. 22 . 
Figure 25: Error measured in the $L^{2}$-norm of energy vs. mesh parameter for refinement sequences with inconsistent geometric map. All refinement sequences start from $C^{0}$ initial meshes except as indicated for $\mathcal{S}_{5}^{1,2}$ and $\mathcal{S}_{5}^{2,3}$ which is $C^{1}$ and $C^{2}$ respectively. Note that the convergence rates for such two refinement sequences are closer to the optimal rates than other refinement sequences, since the geometric map do not change as much as when start refining from $C^{0}$ meshes (see Figure 11). This is another evidence showing the change of geometric map is affecting the convergence rates. The convergence rates are remarkably lower than Figure 23.

One of the major advantages of using triangular meshes in analysis is the ease of local refinement of meshes. We use the Rivara method [54] which uses the element-wise strain energy error to guide the local refinement. The elements with large error are bisected across one of their edges. The local refinement of $\mathcal{S}_{3}^{1}\left(\widehat{T}_{c t}\right)$ mesh is shown in Figure 26. The comparison of error measured in the $L^{2}$-norm of stress vs. degrees of freedom between uniform and adaptive refinement is shown in Figure 27. Clearly the local refinement exhibits superior advantage over uniform refinement by leading to the same accurate results with fewer degrees of freedom. Specifically, to obtain the same order of error, the uniform refinement requires about three times the degrees of freedom as much as the adaptive refinement. 


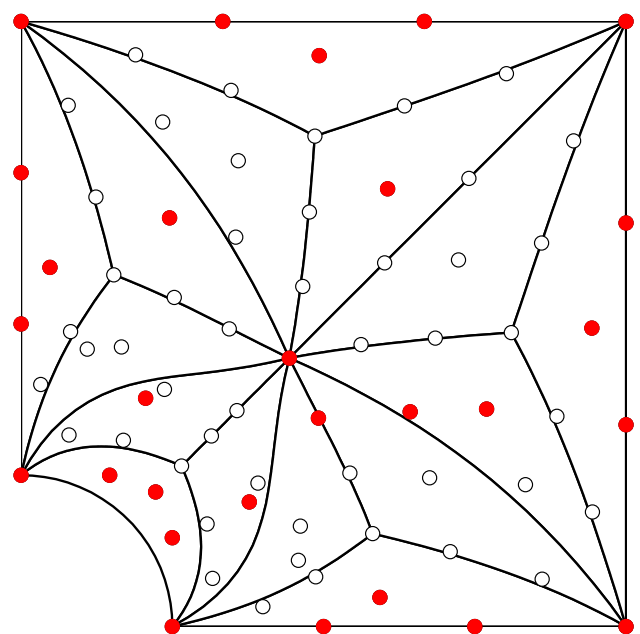

(a) Initial $\mathcal{S}_{3}^{1}\left(\widehat{T}_{c t}\right)$ mesh.

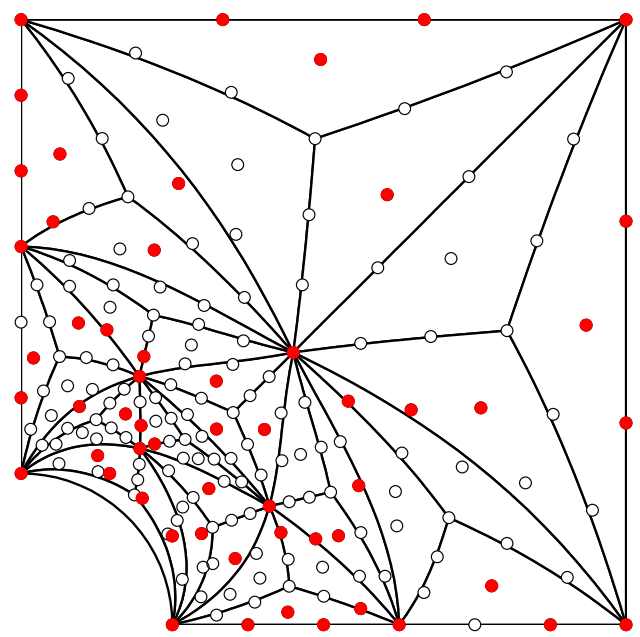

(c) Local refinement 2 .

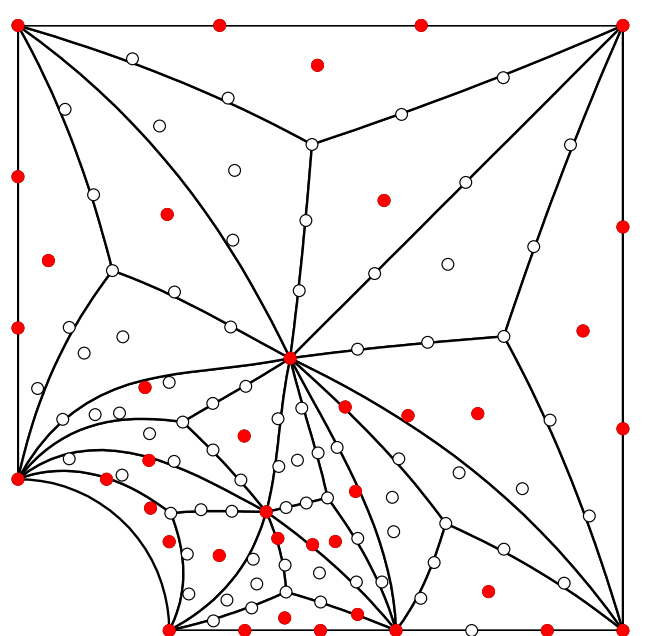

(b) Local refinement 1 .

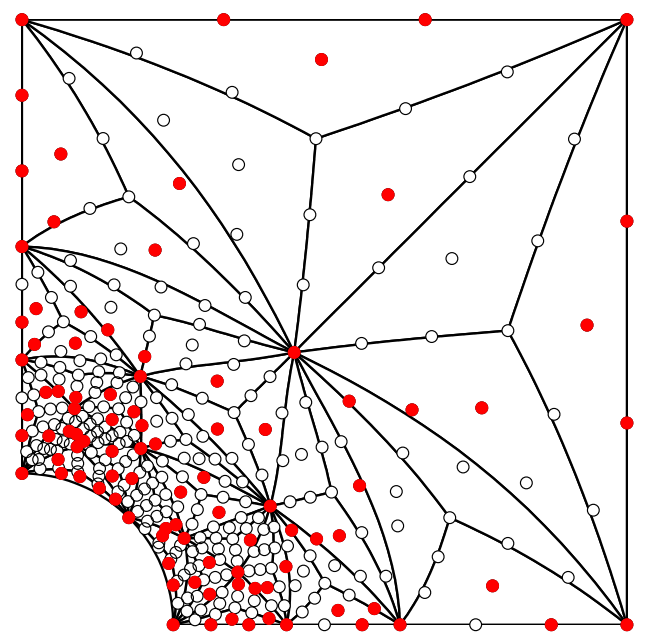

(d) Local refinement 3 .

Figure 26: Local refinement of $\mathcal{S}_{3}^{1}\left(T_{c t}\right)$ mesh.

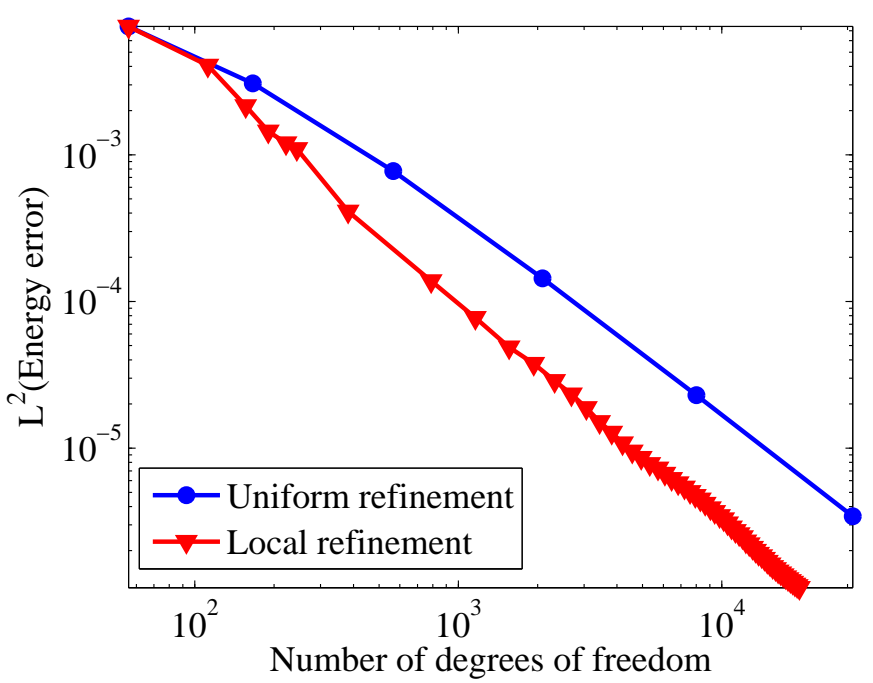

Figure 27: Comparison of error per degree of freedom between uniform and local refinement in space $\mathcal{S}_{3}^{1}\left(T_{c t}\right)$ for the plate hole problem. The local refinement sequence shows superior advantage with same error obtained using only one third of degrees of freedom of uniform refinement. 


\section{Conclusion}

In this paper, we presented a smooth-refine-smooth approach to rTBS based isogeometric analysis that can achieve optimal convergence rates for all $C^{r}$ rTBS elements. For a given NURBS bounded domain with arbitrary topology, the rTBS based parametrization can be fully automated. Various sets of globally $C^{r}$ continuous basis can be constructed by imposing continuity constraints on adjoining triangle elements, through either DC or GE method. Error estimate indicates the constructed $C^{r}$ space delivers optimal convergence rates, provided the geometric map remains the same during refinement.

In order to overcome the inconsistency of geometric map during the refine-then-smooth approach, we have introduced a strategy to construct a pre-refinement map that possesses sufficient continuity for all subsequent refinements. Thus the relocation of control points is avoided and the map stays unchanged during refinement. By constructing such a prerefinement geometric map with sufficient smoothness, optimal convergences have been achieved in all elements. We gave specific smoothness conditions for the pre-refinement geometric map: that should be $C^{r}$ smooth for regular $C^{r}$ elements and be $C^{\rho}$ smooth in cases of superspline spaces $S_{d}^{r, \rho}, \rho>r$ where supersmoothness occurs at the vertices or edges of macro-triangles. Numerical results verified that convergence rates are optimal in different spaces with the introduction of such smooth pre-refinement maps. This demonstrates that $C^{r}$ rTBS elements possess superior efficiency on a per-node basis over $C^{0}$ elements. Such nodal efficiency is especially pronounced in the case of supersplines.

In our proposed smooth-refine-smooth approach, the smoothness in the pre-refinement geometric map is needed to keep the geometric map consistent during the refinement. The smoothness in the last step is used to obtain a stable $C^{r}$ basis for analysis. Future work would look into if the two steps can be combined.

We believe the smooth-refine-smooth approach could also shed light on how to obtain optimal convergence in isogeometric analysis with T-splines when extraordinary points are involved. To obtain $G^{1}$ continuity at the extraordinary points of T-splines, a constrained optimization problem is usually solved to relocate the surrounding control points. This relocation would also change the geometric map as in rTBS based isogeometric analysis. Thus it would be interesting to see if the strategy to obtain consistent geometric map during the $h$-refinement can be similarly applied in T-splines for achieving optimal convergence rates.

Our rTBS based isogeometric analysis approach is general in the sense that any form of $C^{r}$ Bézier elements can be used. It would be interesting to apply normalized B-spline basis functions over triangulations such as [25, 34, 37, 35, 27, 36] in this rTBS based IGA framework since they have desirable properties such as partition of unity and nonnegativeness.

\section{Acknowledgments}

The authors acknowledge the financial support from NSF grant \#1435072. The authors would like to thank Noah Jaxon for his contribution in our earlier work of isogeometric analysis on triangulations [38].

\section{References}

[1] T. Hughes, J. Cottrell, Y. Bazilevs, Isogeometric analysis: CAD, finite elements, NURBS, exact geometry and mesh refinement, Computer Methods in Applied Mechanics and Engineering 194 (39) (2005) 4135-4195. 
[2] J. Cottrell, T. Hughes, A. Reali, Studies of refinement and continuity in isogeometric structural analysis, Computer Methods in Applied Mechanics and Engineering 196 (41) (2007) 4160-4183.

[3] W. A. Wall, M. A. Frenzel, C. Cyron, Isogeometric structural shape optimization, Computer Methods in Applied Mechanics and Engineering 197 (33) (2008) 29762988.

[4] X. Qian, Full analytical sensitivities in NURBS based isogeometric shape optimization, Computer Methods in Applied Mechanics and Engineering 199 (29) (2010) 2059-2071.

[5] X. Qian, O. Sigmund, Isogeometric shape optimization of photonic crystals via Coons patches, Computer Methods in Applied Mechanics and Engineering 200 (25) (2011) $2237-2255$.

[6] J. Cottrell, A. Reali, Y. Bazilevs, T. Hughes, Isogeometric analysis of structural vibrations, Computer Methods in Applied Mechanics and Engineering 195 (41) (2006) $5257-5296$.

[7] Y. Bazilevs, I. Akkerman, Large eddy simulation of turbulent Taylor-Couette flow using isogeometric analysis and the residual-based variational multiscale method, Journal of Computational Physics 229 (9) (2010) 3402-3414.

[8] K. Li, X. Qian, Isogeometric analysis and shape optimization via boundary integral, Computer-Aided Design 43 (11) (2011) 1427-1437.

[9] D. Burkhart, B. Hamann, G. Umlauf, Isogeometric finite element analysis based on Catmull-Clark: subdivision solids, in: Computer Graphics Forum, Vol. 29, Wiley Online Library, 2010, pp. 1575-1584.

[10] T. W. Sederberg, J. Zheng, A. Bakenov, A. Nasri, T-splines and T-NURCCs, in: ACM Transactions on Graphics (TOG), Vol. 22, ACM, 2003, pp. 477-484.

[11] T. W. Sederberg, D. Cardon, G. Finnigan, N. North, J. Zheng, T. Lyche, T-spline simplification and local refinement, in: ACM Transactions on Graphics (TOG), Vol. 23, ACM, 2004, pp. 276-283.

[12] Y. Bazilevs, V. Calo, J. Cottrell, J. Evans, T. Hughes, S. Lipton, M. Scott, T. Sederberg, Isogeometric analysis using T-splines, Computer Methods in Applied Mechanics and Engineering 199 (5) (2010) 229-263.

[13] X. Li, M. Scott, Analysis-suitable T-splines: characterization, refineability, and approximation, Mathematical Models and Methods in Applied Sciences 24 (06) (2014) $1141-1164$.

[14] M. Scott, X. Li, T. Sederberg, T. Hughes, Local refinement of analysis-suitable T-splines, Computer Methods in Applied Mechanics and Engineering 213 (2012) $206-222$.

[15] X. Li, J. Zheng, T. Sederberg, T. Hughes, M. Scott, On linear independence of Tspline blending functions, Computer Aided Geometric Design 29 (1) (2012) 63-76. 
[16] M.-J. Lai, Scattered data interpolation and approximation using bivariate $C^{1}$ piecewise cubic polynomials, Computer Aided Geometric Design 13 (1) (1996) 81-88.

[17] M. Lai, P. Wenston, Bivariate splines for fluid flows, Computers \& fluids 33 (8) (2004) 1047-1073.

[18] G. Awanou, M.-J. Lai, P. Wenston, The multivariate spline method for scattered data fitting and numerical solutions of partial differential equations, Wavelets and splines: Athens (2005) 24-74.

[19] X. Hu, D. Han, M. Lai, Bivariate splines of various degrees for numerical solution of partial differential equations, SIAM Journal on Scientific Computing 29 (3) (2007) $1338-1354$.

[20] L. L. Schumaker, Computing bivariate splines in scattered data fitting and the finiteelement method, Numerical Algorithms 48 (1-3) (2008) 237-260.

[21] M. Powell, M. Sabin, Piecewise quadratic approximations on triangles, ACM Transactions on Mathematical Software (TOMS) 3 (4) (1977) 316-325.

[22] P. Sablonnière, Error bounds for Hermite interpolation by quadratic splines on an $\alpha$-triangulation, IMA Journal of Numerical Analysis 7 (4) (1987) 495-508.

[23] P. Dierckx, S. Van Leemput, T. Vermeire, Algorithms for surface fitting using powellsabin splines, IMA Journal of numerical analysis 12 (2) (1992) 271-299.

[24] K. Willemans, P. Dierckx, Surface fitting using convex powellsabin splines, Journal of Computational and Applied Mathematics 56 (3) (1994) 263-282.

[25] P. Dierckx, On calculating normalized Powell-Sabin B-splines, Computer Aided Geometric Design 15 (1) (1997) 61-78.

[26] C. Manni, P. Sablonniere, Quadratic spline quasi-interpolants on Powell-Sabin partitions, Advances in Computational Mathematics 26 (1-3) (2007) 283-304.

[27] H. Speleers, Multivariate normalized Powell-Sabin B-splines and quasi-interpolants, Computer Aided Geometric Design 30 (1) (2013) 2-19.

[28] H. Speleers, P. Dierckx, S. Vandewalle, Numerical solution of partial differential equations with Powell-Sabin splines, Journal of Computational and Applied Mathematics 189 (1) (2006) 643-659.

[29] H. Speleers, C. Manni, F. Pelosi, M. Sampoli, Isogeometric analysis with PowellSabin splines for advection-diffusion-reaction problems, Computer Methods in Applied Mechanics and Engineering 221 (2012) 132-148.

[30] H. Speleers, C. Manni, F. Pelosi, From NURBS to NURPS geometries, Computer Methods in Applied Mechanics and Engineering 255 (2013) 238-254.

[31] L. Beirão Da Veiga, T. Hughes, J. Kiendl, C. Lovadina, J. Niiranen, A. Reali, H. Speleers, A locking-free model for Reissner-Mindlin plates: Analysis and isogeometric implementation via NURBS and triangular NURPS, Mathematical Models and Methods in Applied Sciences 25 (08) (2015) 1519-1551. 
[32] H. Speleers, C. Manni, Optimizing domain parameterization in isogeometric analysis based on powell-sabin splines, Journal of Computational and Applied Mathematics 289 (2015) 68-86.

[33] M. Lai, L. Schumaker, Spline Functions on Triangulations, Vol. 110, Cambridge University Press, 2007.

[34] H. Speleers, A normalized basis for quintic Powell-Sabin splines, Computer Aided Geometric Design 27 (6) (2010) 438-457.

[35] H. Speleers, Construction of normalized B-splines for a family of smooth spline spaces over Powell-Sabin triangulations, Constructive Approximation 37 (1) (2013) 41-72.

[36] E. Cohen, T. Lyche, R. Riesenfeld, A B-spline-like basis for the Powell-Sabin 12-split based on simplex splines, Mathematics of Computation 82 (283) (2013) 1667-1707.

[37] H. Speleers, A normalized basis for reduced Clough-Tocher splines, Computer Aided Geometric Design 27 (9) (2010) 700-712.

[38] N. Jaxon, X. Qian, Isogeometric analysis on triangulations, Computer-Aided Design 46 (2014) 45-57.

[39] Y. Bazilevs, L. Beirão da Veiga, J. Cottrell, T. Hughes, G. Sangalli, Isogeometric analysis: approximation, stability and error estimates for h-refined meshes, Mathematical Models and Methods in Applied Sciences 16 (07) (2006) 1031-1090.

[40] L. Beirão da Veiga, A. Buffa, D. Cho, G. Sangalli, IsoGeometric analysis using T-splines on two-patch geometries, Computer Methods in Applied Mechanics and Engineering 200 (21) (2011) 1787-1803.

[41] M. Scott, T-splines as a Design-Through-Analysis technology, Ph.D. Thesis, The University of Texas at Austin.

[42] M. Scott, R. Simpson, J. Evans, S. Lipton, S. Bordas, T. Hughes, T. W. Sederberg, Isogeometric boundary element analysis using unstructured T-splines, Computer Methods in Applied Mechanics and Engineering 254 (2013) 197-221.

[43] G. Farin, Curves and surfaces for CAGD: a practical guide, Morgan Kaufmann, 2002.

[44] D. Hong, Spaces of bivariate spline functions over triangulation, Approx. Theory Appl 7 (1) (1991) 56-75.

[45] R. Clough, J. Tocher, Finite element stiffness matrices for analysis of plate bending, 1966. (1966) 515-545.

[46] K. Stein, T. Tezduyar, R. Benney, Mesh moving techniques for fluid-structure interactions with large displacements, Journal of Applied Mechanics 70 (1) (2003) $58-63$.

[47] P. Alfeld, Bivariate spline spaces and minimal determining sets, Journal of Computational and Applied Mathematics 119 (1) (2000) 13-27. 
[48] M. J. Borden, M. A. Scott, J. A. Evans, T. J. R. Hughes, Isogeometric finite element data structures based on Bézier extraction of NURBS, International Journal for Numerical Methods in Engineering 87 (1-5) (2011) 15-47.

[49] M. A. Scott, M. J. Borden, C. V. Verhoosel, T. W. Sederberg, T. J. R. Hughes, Isogeometric finite element data structures based on Bézier extraction of T-splines, International Journal for Numerical Methods in Engineering 88 (2) (2011) 126-156.

[50] J. Lyness, R. Cools, A survey of numerical cubature over triangles, in: Proceedings of Symposia in Applied Mathematics, Vol. 48, 1994, pp. 127-150.

[51] D. Wang, J. Xuan, An improved NURBS-based isogeometric analysis with enhanced treatment of essential boundary conditions, Computer Methods in Applied Mechanics and Engineering 199 (37) (2010) 2425-2436.

[52] D. Thomas, M. Scott, J. Evans, K. Tew, E. Evans, Bézier projection:a unified approach for local projection and quadrature-free refinement and coarsening of NURBS and T-splines with particular application to isogeometric design and analysis, Computer Methods in Applied Mechanics and Engineering 284 (2015) 55-105.

[53] P. Gould, Introduction to Linear Elasticity, Springer, 1983.

[54] M. Rivara, New mathematical tools and techniques for the refinement and/or improvement of unstructured triangulations, in: Proc. 5th Int. Meshing Roundtable, Vol. 96, Citeseer, 1996, pp. 77-86. 